\title{
A Study of Particle Surface Effects on the Spall Strength of Particle-Reinforced Polymer Matrix Composites
}

by

\author{
Anton Jacob Lebar \\ A thesis submitted to the \\ Faculty of Graduate and Postdoctoral Affairs \\ in partial fulfillment of the requirements for the degree of
}

Master of Applied Science in Mechanical Engineering

Ottawa-Carleton Institute for Mechanical and Aerospace Engineering

Department of Mechanical and Aerospace Engineering

Carleton University

Ottawa, Ontario

June, 2020

(C)Copyright

Anton Jacob Lebar, 2020 
The undersigned hereby recommends to the

Faculty of Graduate and Postdoctoral Affairs acceptance of the thesis

\title{
A Study of Particle Surface Effects on the Spall Strength of Particle-Reinforced Polymer Matrix Composites
}

\author{
submitted by Anton Jacob Lebar \\ in partial fulfillment of the requirements for the degree of \\ Master of Applied Science in Mechanical Engineering
}

Professor Oren E. Petel, Thesis Supervisor

Professor Huseyin Gunay, CEE

Professor Henry Saari, MAE

Professor Patrick Dumond, UOttawa

Professor Ronald Miller, Chair, MAE

Carleton University

June, 2020 


\section{Abstract}

In the field of shock physics, flat plate impacts are used to characterize the dynamic tensile strength (spall strength) of a material. In particular, prior work has observed that the presence of secondary phases influences the overall material, typically reducing spall strength. In the study presented here, surface effects were investigated using polymer matrix composites reinforced with alumina particles. Interface adhesion between the matrix and filler was tailored with silanes, while filler morphology effects were assessed using spherical and irregular particle geometries. Two matrix materials were used, polydimethylsiloxane and polyurethane. These composites were characterized through spall experiments, using a single stage light gas gun at Carleton University. Varying particle morphology was found to have no effect on spall strength, while composites with impeded adhesion saw a drastic reduction in spall strength, associated with a loss of adhesion at the interface. 


\section{Acknowledgments}

While the road to get here has been long and sometimes difficult, I sincerely recognize the vast support provided by those around me. Without their support and guidance, I do not believe I would have made it to this point.

I would like to begin by acknowledging the guidance and wisdom imparted to me, by my supervisor Professor Oren Petel. By luck and circumstance, our time as colleagues started long ago, when I was but a humble second-year undergraduate research assistant. From that moment on, you have been my mentor, providing guidance, and insight into every challenge that research has thrown at me.

In my time as a graduate student, several caring individuals in the Department of Mechanical and Aerospace Engineering have supported my work, from behind the scenes. To Alex Proctor, Kevin Sangster, and Aric Adock. Without your expertise and experience, I have no doubt I'd be still designing the countless gauges, molds, and widgets you've manufactured for me over the last few years. To Steve Truttman, David Raude, Stephan Biljan, and James Cann, your technical knowledge and ready assistance in the lab paved the way to success in my experiments. To the administrative staff in the MAE Departmental office; thank you all, for the entertaining discussions, and your assistance with administrative hurdles.

To my family, thank you to everyone. To my parents (Adrian and Sabrina) and stepmother (Sarah), you provided the support, confidence, and guidance I needed to get to this point. To my grandparents (Nana, Dedek, and Oma), thank you for always being so proud of me, and making me feel so special. To my siblings (Aidon, Jordan, Rowan, and Aaron), thank you for being lovable goof balls, each in your own special way. You always made me 
smile, or gave me a laugh when I needed it. The enduring support of my family pushed me forward every step of the way. You have all said I would go into engineering, ever since I was a little boy. Well it looks like I did, not once, but twice.

To Scott and Ashley, thank you for joining me on this challenging journey. I don't think I would have made it without the beers or rants we shared. It's been a long time coming, but we all did it. To all of the other graduate students with whom I have shared an office, I want to thank you for listening to me mumble on about polymers, lasers, spall strengths, gas guns, and coil gauges. I may have sounded crazy, but I hope this thesis proves otherwise.

To all my friends, you have my undying gratitude. Thank you for not saying anything when I gave the oft-repeated "It's almost there, I'm just writing my thesis, I'll be done soon". To Julia, thank you for tolerating me. You have been amazing during such a turbulent chapter in my life. You were always there to hear me rant and complain about that day's frustrations. You always supported me and pushed me towards this finish line.

I also acknowledge the generous financial support received from the Natural Science and Engineering Research Council of Canada through the Discovery Grant, and the Research Tools and Instruments programs to support this research. Financial support provided by the Department of National Defence through the Innovation for Defence Excellence and Security (IDEaS) program is also greatly appreciated.

Throughout my life I have been told countless times "Close only counts in horse shoes and hand grenades". Lucky for me, the field of shock physics is thoroughly ensconced in the latter of the two. 


\section{Table of Contents}

Abstract

Acknowledgments $\quad$ iv

Table of Contents $\quad$ vi

List of Figures $\quad$ x

List of Tables $\quad$ xiv

Nomenclature $\quad$ Xv

1 Introduction 1

1.1 Introduction to Spall $\ldots \ldots \ldots \ldots \ldots$

1.2 Current Study . . . . . . . . . . . . . . . . . . . 4

2 Literature Review 6

2.1 Spall in Metals . . . . . . . . . . . . . . . . . 6

2.2 Spall in Polymers . . . . . . . . . . . . . . . . . 10

3 Wave Mechanics $\quad 16$

3.1 Compressibility and Shock Waves . . . . . . . . . . . . . . . . . 16

3.2 Wave Interactions during a High Strain Rate Plate Impact . . . . . . . . . . 20

3.2 .1 Initial Impact . . . . . . . . . . . . . . . . . . 21

3.2.2 Transmission of Waves between Mediums . . . . . . . . . . . . . 23 
3.2.3 Arrival of a Wave at a Free Surface . . . . . . . . . . . . . . . 24

3.3 Spall Mechanics . . . . . . . . . . . . . . . . . . . 25

3.4 Spall Strength . . . . . . . . . . . . . . . . . . . . . . . . . 29

3.4.1 Acoustics and Composites . . . . . . . . . . . 30

4 Experimental Details $\quad 33$

4.1 Sample Preparation . . . . . . . . . . . . . . . . . . 34

4.1.1 Filler Treatment with Silane . . . . . . . . . . . . . . 35

4.1.2 Preparation of Polyurethane Samples . . . . . . . . . . . . . 36

4.1.3 Preparation of PDMS Samples . . . . . . . . . . . . . . 37

4.2 Single Stage Light Gas Gun . . . . . . . . . . . . . . . . . 38

4.3 Plate Impact Testing $\ldots \ldots \ldots$. . . . . . . . . . . . . . 40

4.3.1 Sound Speed Measurements . . . . . . . . . . . . . . . . 42

4.3.2 Geometry of the Sabot and Flyer Plate . . . . . . . . . . . . . . 43

4.3.3 Target Assembly . . . . . . . . . . . . . . . . . 46

4.4 Experimental Setup of the PDMS Study . . . . . . . . . . . . . . . . . 48

4.5 Test Matrix . . . . . . . . . . . . . . . . . . . 50

4.6 Instrumentation and Data Processing . . . . . . . . . . . . 51

4.6.1 Annular Coil Gauges . . . . . . . . . . . . . . . . 51

4.6.2 Photonic Doppler Velocimetry . . . . . . . . . . . . . . 52

5 Results and Discussion $\quad 56$

5.1 Spall in PDMS Systems . . . . . . . . . . . . . . . . . 56

5.2 Polyurethane Spall Testing . . . . . . . . . . . . . . . 61

5.2.1 Sound Speed and Density Measurements . . . . . . . . . . . 62

5.2.2 Spall in Neat Polyurethane . . . . . . . . . . . . . . . 66

5.2.3 Effect of Particle Reinforcement on Spall Strength . . . . . . . . . 70 
5.2.4 Effect of Particle Morphology on Spall Strength . . . . . . . . . . . 71

5.2.5 Interpretation of Spall Strength in Negatively Treated Samples . . . 74

5.2.6 Interface Adhesion Effects on Spall Strength . . . . . . . . . . . . 77

5.2 .7 Silane Polyurethane Interactions . . . . . . . . . . . . . . . . . 81

5.2 .8 Fracture Surface Analysis . . . . . . . . . . . . . 84

$\begin{array}{llr}6 & \text { Conclusion } & 88\end{array}$

6.1 Future Work . . . . . . . . . . . . . . . . . . . . . . . . . . . . 89

$\begin{array}{ll}\text { List of References } & 92\end{array}$

$\begin{array}{lll}\text { Appendix A } & \text { Tabulated Results } & 99\end{array}$

$\begin{array}{lll}\text { Appendix B Spectrograms } & 101\end{array}$

$\begin{array}{lll}\text { Appendix C SEM Imaging } & 111\end{array}$

C.1 Imaging of the Neat Samples . . . . . . . . . . . . . . . . . . . 112

C.2 Imaging of the Pristine Spherical Samples . . . . . . . . . . . . 113

C.3 Imaging of the Pristine Irregular Samples . . . . . . . . . . . . . 115

C.4 Imaging of the Silane Treated Spherical Samples _ . . . . . . . . . . 117

C.5 Imaging of the Polyurethane-Silane Blended Samples . . . . . . . . . . . 119

$\begin{array}{lll}\text { Appendix D } & \text { Silane Chemistry } & 121\end{array}$

$\begin{array}{lll}\text { Appendix E } & \text { Data Processing Scripts } & 123\end{array}$

E.1 Read Lecroy Oscilliscope . . . . . . . . . . . . . . . . . . . . . . . 123

E.2 Processing of Shock pin Data . . . . . . . . . . . . . . . . . . 124

E.3 Processing of Coil Gauge Data . . . . . . . . . . . . . 126

E.4 Photo Doppler Velocimetry Processing . . . . . . . . . . . . . . . . . . . 127 
E.5 Design of spall experiments . . . . . . . . . . . . . . . . . . 131

E.5.1 P_u_Interface . . . . . . . . . . . . . . . . 135

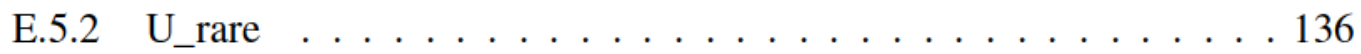




\section{List of Figures}

1.1 Molecular dynamics simulation showing the formation of a spall plane over time, as a result of a flat plate impact . . . . . . . . . . . . 2

1.2 Post impact images showing the spall plane in sectioned (a) polyurethane and (b) steel samples . . . . . . . . . . . . . . . . . . . . . 3

2.1 The energy-time plot for spall in a ductile material denoting the minimum time threshold for energy limited and subsequent flaw limited spall . . . . . 8

2.2 SEM imaging of armour grade aluminum post spall, with the arrows denoting debonding of the inter-metallic particles from the aluminium matrix . . 9

2.3 SEM imaging of epoxy-CNT composite post spall, showcasing nanotube pullout from the matrix . . . . . . . . . . . . . . . . 12

3.1 Control Volume Enclosing a Wave . . . . . . . . . . . . . . . . . 16

3.2 Representation of (a) the velocity along a wave and the (b) "shocking up" of the wave with the progression of time . . . . . . . . . . . . 18

3.3 The separation of a rarefaction fan and its overtaking of a shockwave . . . . 20

3.4 Wave structure of a plate impact experiment . . . . . . . . . . . . . 21

3.5 Schematic depicting the impact of two semi-infinite slabs . . . . . . . 22

3.6 $P-u_{p}$ Hugoniot depicting an initial impact between two materials . . . . . 22

3.7 $P-u_{p}$ Hugoniot depicting the arrival of a wave at an interface between two materials ........................ 23

3.8 $P-u_{p}$ Hugoniot depicting the unloading of a material via the arrival of a shock wave at a free surface . . . . . . . . . . . . . . . . . . 24

3.9 (a) Schematic and (b) x-t diagram of Newton's cradle in operation. . . . . . 26 
3.10 (a) Schematic and (b) x-t diagram of spall in a flat plate impact. . . . . . . 26

3.11 a) $x-t$ diagram showing generation of spall plane in a target (b) same $x-t$ diagram as in (a) zoomed to region where the rarefaction fans overlap . . . 27

3.12 (a) $P-u_{p}$ Hugoniot depicting the development of tension during spallation of a target (b) Same Hugoniot as in (a) zoomed to focus on the negative

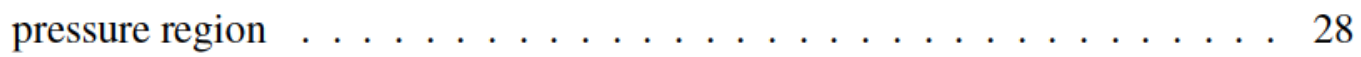

3.13 General back face velocity schematic during spall in neat polyurethane . . . 29

4.1 SEM images of the (a) five $\mu \mathrm{m}$ spherical alumina and (b) 1000 grit irregular alumina powders used in this study . . . . . . . . . . . . . . . . . 34

4.2 Schematics depicting the (a) aliphatic terminated (SIO6715.0) and (b) acrylate terminated (SIM6487.3) silane molecules used in this study . . . . 36

4.3 Schematic of the gas gun, showing the breech, barrel, and catch tank regions 40

4.4 Schematic of instrumented sample mounted to the coil gauges on the muzzle of the gas gun . . . . . . . . . . . . . . . . 41

4.5 (a) Olympus M202-RM linear transducer and (b) Olympus 45 MG thickness gauge . . . . . . . . . . . . . . . . . . . . . . 42

4.6 Labelled schematic of the sabot and flyer plate assembly . . . . . . . . . 43

4.7 (a) Schematic and (b) plot for quantifying yaw of the projectile as a function of projectile length . . . . . . . . . . . . . . . . . . . . 44

4.8 Observation of a clear spall plane in a recovered polyurethane sample . . . 45

4.9 Target assembly mounted to the muzzle of the barrel and instrumented with two PDV probes ........................ 46

4.10 Schematic representations of the experimental apparatus used for the polyurethane based samples; (a) labelled side view and (b) isometric view . 47

4.11 Schematic representations of the experimental apparatus used for the PDMS based samples; (a) labelled side view and (b) isometric view . . . . 48 
4.12 Shock Pin trace of a PDMS spall experiment corresponding to a tilt of 2.8 milli-radians . . . . . . . . . . . . . . . . . . . . . . . . . 49

4.13 (a) Laser velocimetry and (b) coil gauge instrumentation observing the same projectile, recording a velocity of $263 \mathrm{~m} / \mathrm{s}$ and $259 \mathrm{~m} / \mathrm{s}$ respectively. . 53

4.14 Schematic of PDV setup . . . . . . . . . . . . . 53

4.15 (a) Raw PDV data as displayed on our oscilloscope compared and its (b) free surface velocity spectrogram after FFT processing . . . . . . . . . . . 54

5.1 (a) PDMS-Alumina target struck by an acrylic flyer at $282 \mathrm{~m} / \mathrm{s}$ (b) same impact as shown in (a) cropped to focus on peak and pullback velocities . . 57

5.2 (a) Positively-treated PDMS-Alumina target struck by an acrylic flyer at $312 \mathrm{~m} / \mathrm{s}$ (b) same impact as shown in (a) cropped to focus on peak and pullback velocities . . . . . . . . . . . . . . 58

5.3 (a) Negatively-treated PDMS-Alumina target struck by an acrylic flyer at $283 \mathrm{~m} / \mathrm{s}$ (b) same impact as shown in (a) cropped to focus on peak and pullback velocities . . . . . . . . . . . . . . . . 59

5.4 Spectrograms of spall experiments showing: (a) resonance, and (b) the noise generate by ejecta from the back face . . . . . . . . . . . 60

5.5 PDV spectrogram suggesting the ejection of the aluminized Mylar from the back face of a sample . . . . . . . . . . . . . . . . . . . . . 61

5.8 Experimental spall strength of the neat polyurethane as a function of strain

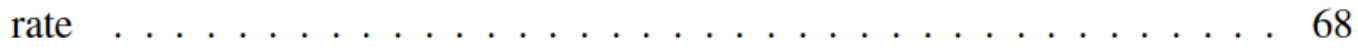

5.10 Experimental spall strength measurements plotted against strain rate of the alumina reinforced PU composites showing the effect of second phase particles ......................... . 70

5.12 Free surface velocity spectrogram of a silane treated polyurethane sample showing the two candidates for minimum particle velocity $\ldots \ldots . . .75$ 
5.13 (a) Spall traces of particle filled elastomeric rocket propellant simulants (b) and rubber $\ldots \ldots \ldots \ldots \ldots \ldots \ldots$

$5.14 \mathrm{x}$-t diagram of spall in a negatively treated sample struck at $400 \mathrm{~m} / \mathrm{s} \ldots 77$

5.15 Experimental spall strength measurements plotted against strain rate of the alumina reinforced PU composites showing the effect of impeded interface adhesion . . . . . . . . . . . . . . . . . 78

5.16 (a) Cross-sectional photo of silane-treated, $20 \%$ alumina-reinforced PU sample showing an absence of a clear spall plane. (b) Zoomed image to to show a magnified portion of the cross-section presented in (a) . . . . . 79

5.17 (a) Clear spall plane formation in a silane-treated, $20 \%$ alumina-reinforced PU sample. (b) PDV spectrogram of the sample in (a) during the spall

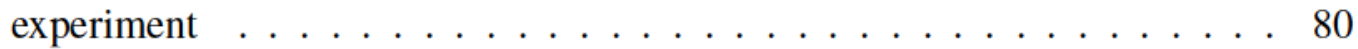

5.18 Experimental spall strength measurements of the PU and PU+silane blend plotted against strain rate . . . . . . . . . . . . . . . 81

5.19 (a) Neat polyurethane target struck by an acrylic flyer at $290 \mathrm{~m} / \mathrm{s}$ (b) polyurethane silane blend target struck by an acrylic flyer at $292 \mathrm{~m} / \mathrm{s}$. . . . 82

5.20 Images of recovered PU silane spall samples: (a) 1/8" acrylic impactor at $281 \mathrm{~m} / \mathrm{s}$, (b) $1 / 8$ " aluminum impactor at $290 \mathrm{~m} / \mathrm{s} \ldots \ldots$. . . . . . . . . . . 83

5.21 A selection of SEM images displaying the fracture surface of a spherical alumina reinforced PU sample . . . . . . . . . . . . . . . . . . . 84

5.22 A selection of SEM images displaying the fracture surface of an irregular alumina reinforced PU sample . . . . . . . . . . . . . . . . . . . 85

5.23 A selection of SEM images displaying the fracture surface of a silanetreated, spherical alumina reinforced PU sample . . . . . . . . . . . . . 86 


\section{List of Tables}

4.1 Particle size distributions of the alumina filler used in this study . . . . . 35

4.2 Test matrix of the PDMS study . . . . . . . . . . . . . 50

4.3 Test matrix of the polyurethane study $\ldots \ldots \ldots \ldots$. . . . . . . 51

5.1 Results of plate impact testing of PDMS composites . . . . . . . . . . 57

5.2 Average measured and theoretical density of each sample type . . . . . . 62

5.3 Average measured and theoretical sound speeds of each sample type . . . 65

5.4 Estimated Young's modulus of each alumina reinforced sample type . . . . 66

5.5 Comparison of the properties of neat polyurethane and a polyurethane

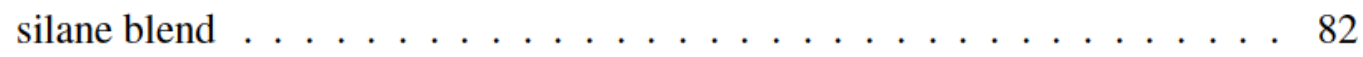




\section{Nomenclature}

\begin{tabular}{|c|c|}
\hline Carbon Nano-tube & CNT \\
\hline Scanning electron microscope & SEM \\
\hline Quasi-static & QS \\
\hline Aspect Ratio & AR \\
\hline improvised explosive device & IED \\
\hline polydimethylsiloxane & PDMS \\
\hline polyurethane & PU \\
\hline control volume & $\mathrm{CV}$ \\
\hline equation of state & EOS \\
\hline right going & RG \\
\hline left going & LG \\
\hline photonic Doppler Velocimeter & PDV \\
\hline Fast Fourier Transform & FFT \\
\hline
\end{tabular}




\section{Chapter 1}

\section{Introduction}

In today's battlefield, soldiers are surviving deployment in ever increasing percentages, due to developments in emergency medicine and hard composite armour systems for torso and head protection [1]. While these systems save lives, amputation and other trauma continue to come from debilitating wounds to extremities and the groin region [1]. Of particular interest, is the onset of these injuries from debris behaving as high velocity ejecta, driven by the detonation of buried charges. Further, the prevalence of injury cases involving buried improvised explosive devices (IEDs) continue to rise, including injuries to the groin and extremities $[2,3]$.

One of the difficulties in armouring the groin and extremities, compared to the torso and head is the fact that a soldier relies on flexibility in these regions, to maintain the mobility required to perform their duties. Contemporary soft armour systems are typically woven aramid systems. While aramid systems have proven successful as a soft armour system against firearms, they have proven susceptible to the aforementioned buried charge ejecta. Improvements to soft armour materials through the addition of interstitial materials may provide a pathway to improve fabric resistance to this threat.

In order to grade these possible vectors for fabric improvement, some testing methodology is required to evaluate these candidates. Unlike low strain rate methodologies, spall and ballistic testing subject materials to the strain rates and loads relevant to armour materials and the threats they serve to protect against. Ballistic testing is a useful, but complicated testing methodology, with many possible failure mechanisms (plugging, piercing, 
etc.) [4]. Areal density, target thickness, and projectile geometry considerations serve to further complicate the design space. High strain rate spall testing, induced by flat plate impact is a simpler methodology, that induces tensile failure under a one-dimensional strain state [5]. This allows for a straightforward assessment of the material in question, under relevant strain rates and loads. For these reasons, spall was selected as the testing methodology, to assess the materials presented in this study.

\subsection{Introduction to Spall}

The definition of spall can carry different meanings, to different readers. To a civil engineer, spall is the ejection of flakes or sheets from the surface of a concrete structure [6]. Most mechanical engineers know spall to be the failure of a rolling bearing, due to subsurface fatigue, beneath the bearing raceway, culminating in the break out of material from the raceway surface [7]. In the shock physics community, spall specifically refers to the dynamic tensile failure of a material. Spall of a material is a consequence of the constructive interference of planar unloading waves [8], resulting in the generation of an internal planar fracture surface, known as the spall plane. Shown in Figure 1.1 is a representation of the creation of a spall plane, formed by plate impact.
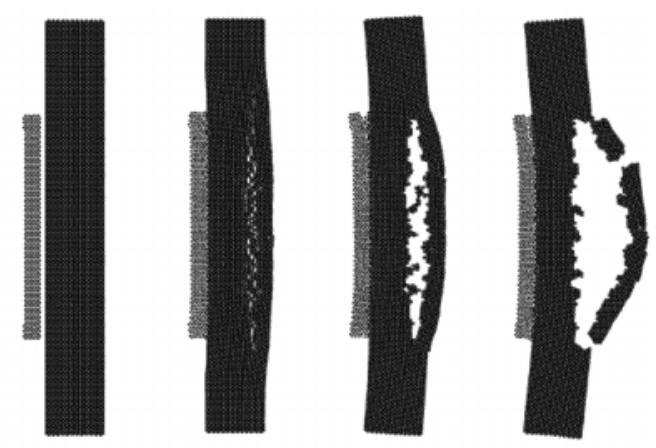

Figure 1.1: Molecular dynamics simulation showing the formation of a spall plane over time, as a result of a flat plate impact [9] 
Depending on the ductility of the medium, failure is the result of the nucleation and coalescence of voids in a ductile material [10], or the formation of micro-cracks that coalesce into larger cracks [11]. Figure 1.2 shows the internal fracture surface from a pair of samples recovered after testing. These samples were subjected to a face to face impact by a high velocity flat plate, in the manner depicted in Figure 1.1.

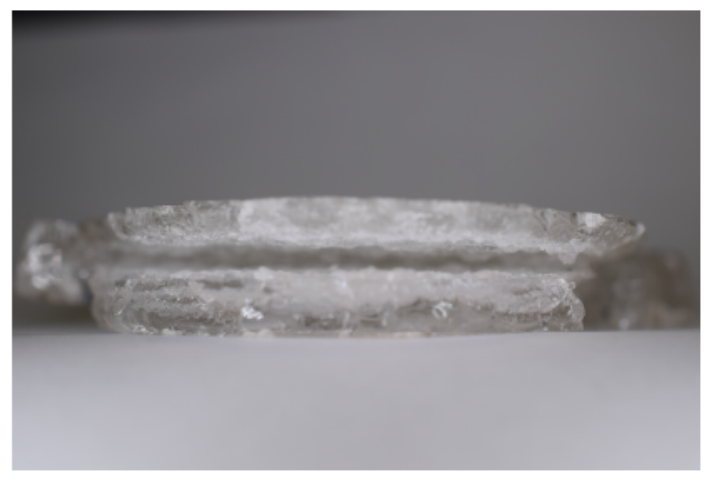

(a)

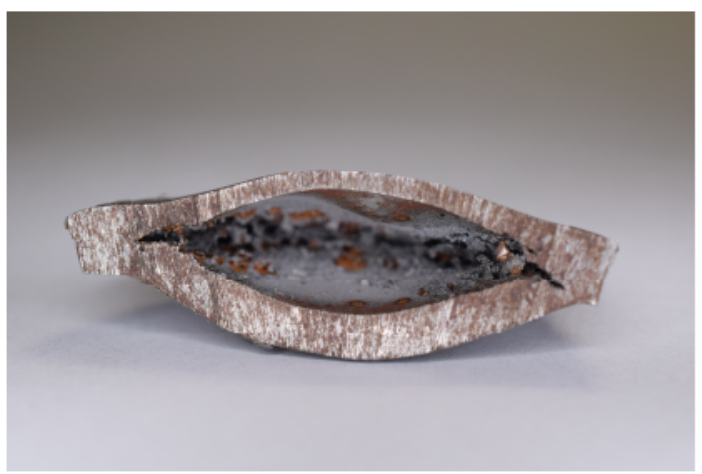

(b)

Figure 1.2: Post impact images showing the spall plane in sectioned (a) polyurethane and (b) steel samples.

Decades of research into the spall strength and failure mechanisms of metals has been performed in the past $[11,12]$, studying the implications of a range of material properties and experimental parameters, including strain rate $[13,14]$, micro-structure $[15,16]$, and the effect of secondary phases $[5,17]$. In particular, prior work investigating the role of secondary phases in dynamic tensile failure in metal alloys has found that interactions involving these secondary phases influences this dynamic behaviour, with evidence that spall failure originated at the phase boundaries, reducing the overall spall strength of the alloy $[5,17]$. Spall strength can be considered equal to the tensile stress state of the material just prior to the onset of spall.

Extending this work to polymers, research has shown that the presence of nanotubes in an epoxy-carbon nanotube (CNT) composite reduces the spall strength, with respect to that 
of the neat epoxy $[18,19]$. Scanning electron microscopy (SEM) observed fibre pullout, at the internal fracture surface, which is indicative of a low interface strength between the CNTs and the epoxy interface. A low strength interface could increase nucleation density, by behaving as the preferred location for void nucleation.

Prior work investigating particle-elastomer composites noted that the surface adhesion between the particles and matrix were important factors in the performance of the composites [20]. Investigations into the ballistic performance of ceramic particle-reinforced polydimethylsiloxane (PDMS) composites showed the alumina reinforced samples exceeded the performance of the silicon carbide and diamond samples, despite lower particle strength, similar densities, and comparable morphologies [20]. It was suggested, that despite the high strain rates and stresses involved, the surface adhesion between the particles and the matrix was an important factor in the ballistic performance.

\subsection{Current Study}

Combining polymer flexibility with the performance of hard ceramic particles into a flexible, ceramic-reinforced polymer matrix composite has been proven to improve ballistic performance [20]. To improve upon existing systems, silanes were used in this study to functionalize micron-scale alumina powder, in order to tailor interface adhesion between the alumina filler and the polymer matrix. Two silanes were selected to increase and decrease interface adhesion respectively, to further observe the significance of interface adhesion. Two polymer matrices, were used in this study, polydimethylsiloxane (PDMS) and polyurethane (PU). PDMS was selected initially, but replaced with PU to improve the overall spall strength of the composites.

In addition to interface adhesion effects, the influence of filler morphology on the spall behaviour of polymer matrix composites has been studied. In this case, a spherical and 
irregular particle shape were used to normalize the filler material, while modifying filler morphology. The size of both particles were kept comparable to mitigate the influence of particle size in this study.

Dynamic tensile failure by flat plate impact was induced in this suite of composites to observe the influence of filler morphology and interface adhesion on the spall strength of the bulk composite. As mentioned above, past studies have observed the effect of filler morphology on ballistic performance [20], while others have speculated on the significance of interface adhesion without direct investigation [19,21]. Therefore, the purpose of this study is to directly investigate the influence of particle morphology and matrix-filler interface adhesion on the spall strength of particle reinforced polymer matrix composites. It is anticipated that these findings will assist in the future design of flexible armours for ejecta protection applications. In addition, this study will further clarify the influence of second phase particles and their interactions on the dynamic behaviour of multi-phase systems.

Next, Chapter 2 will present and discuss relevant literature pertaining to spall, with the specific intent of discussing elements that would influence the performance of the composites presented in this study. Chapter 3 will present the fundamentals of shockwaves, wave interactions, and spall, allowing for an understanding of the spall experiments performed in this study. Chapter 4 provides insight into the apparatuses and methodologies used in this study. Chapter 5 presents the findings of this study, discusses them, and elaborates on their significance. Lastly, Chapter 6 will provide concluding remarks on the work presented here. 


\section{Chapter 2}

\section{Literature Review}

As stated previously, this chapter serves as a discussion regarding pertinent literature, with the objective of providing details that will prove useful in understanding and discussing the findings of this study. This literature review will assess a range of materials including metals, and polymers, under a variety of loading regimes. In this review, attention will be paid to properties and loading conditions that influence material strength and dominate failure processes.

\subsection{Spall in Metals}

Decades of research into the spall strength and failure mechanisms of metals has been performed in the past $[11,12]$, studying the implications of a range of material properties and experimental parameters. In the $1980 \mathrm{~s}$, a lot of work was performed $[15,22]$, to help develop a more fundamental understanding of spall, which culminated in the theory of "energy-limited" and "flaw-limited" spall. Although a high strain rate event, spall is still time dependent, relying on the passage of time for the failure process to occur. This discussion begins with an inequality (Equation 2.1), relating local available kinetic (KE) and elastic energy (PE) to the requisite fracture surface energy (SE) required in ductile spall [22]:

$$
\begin{gathered}
K E+P E \geq S E \\
\frac{1}{2} \frac{P^{2}}{\rho c_{0}^{2}}+\frac{1}{120} \rho \dot{\varepsilon}^{2} s^{2} \geq Y \varepsilon_{c}
\end{gathered}
$$


where $\rho$ is material density, $c_{0}$ is sound speed, $\dot{\varepsilon}$ is strain rate, $s$ is fragment size, $Y$ is flow stress, $\varepsilon_{c}$ is critical void volume fraction [23] required for the coalescence of the void [15]. $P$ is the applied load, which will increase as the material is loaded into tension and is defined as [22]:

$$
P=\rho c_{0}^{2} \dot{\varepsilon} * t
$$

$s$, known as fragment size, is a function of time $(t)$, and is defined by the horizon condition [22]:

$$
s \leq 2 c_{0} * t
$$

From Equations 2.2, 2.3, and 2.4, it can be seen that the elastic and kinetic energy terms will increase over time, with the growth of fragment size $(s)$ and applied load $(P)$. This increase in energy serves as the source of requisite energy to drive the spall event [22]. This means that for spall to occur, a minimum time is required such that the inequality presented in 2.2 is satisfied, and a sufficient amount of energy has been delivered into the development of fracture. When the condition is met, a material with a favourable inherent flaw structure can spall after this minimum time (energy-limited spall). Favourable flaws within a structure are those that support the growth of cracks/voids, such as pre-existing voids/bubbles or inclusions. As depicted in Figure 2.1, a material with a non-favourable flaw structure will spall at a later time, under a higher load (flaw-limited), as energy and time are expended developing smaller, more resistant flaws.

The influences of favourable flaw structures can be seen in case of metals with intermetallic particles, where these secondary phases within metals tend to serve as the dominant location for void nucleation [12]. While homogeneous nucleation through "submicroscopic heterogeneities such as low angle grain boundaries, dislocation tangles/networks, and fine impurities" [24] does occur concurrently in the bulk matrix, void nucleation is 


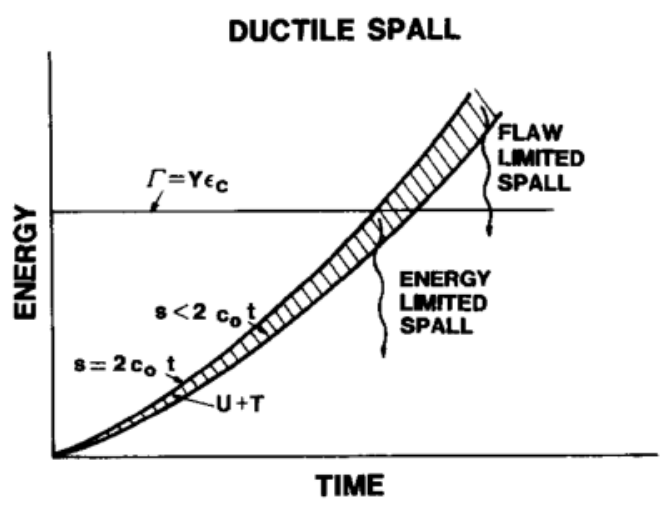

Figure 2.1: The energy-time plot for spall in a ductile material, denoting the minimum time threshold for energy-limited and subsequent flaw-limited spall [22]

dominated by the second-phase particles, through debonding and brittle fracture within the second-phase particle. Examples of second-phase particle driven nucleation can be seen for a number of metals, including AISI 1010 steel with MnS particles, nickel, copper, aluminum [12], and beryllium with $\mathrm{BeO}$ inclusions [24].

In the case of armour grade aluminum alloys, intermetallic secondary phases were deliberately induced through tailored manufacturing processes [17]. Testing observed that the presence of these intermetallic particles reduced the spall strength of the bulk material. Specifically, there was evidence of spall failure originating at the phase boundaries (Figure 2.2). Earlier work found that the spall plane tends to propagate between these inclusions, with cracks initiating or terminating at phase boundaries [5]. Similar work in steels saw that cracks in the steels clearly nucleated at the phase interfaces, then propagated along the interface or through the brittle secondary phase [25]. Furthermore, specimens with an increased interface density saw a reduced spall strength, which was attributed to the higher density of nucleation sites.

Similar findings were seen in metal-matrix composites [26], with larger reductions in spall strength correlating with increasing filler volume fraction. In the case of a metalmetal composite $(\mathrm{Cu} / \mathrm{Nb})$, no difference in spall strength was observed compared to the 


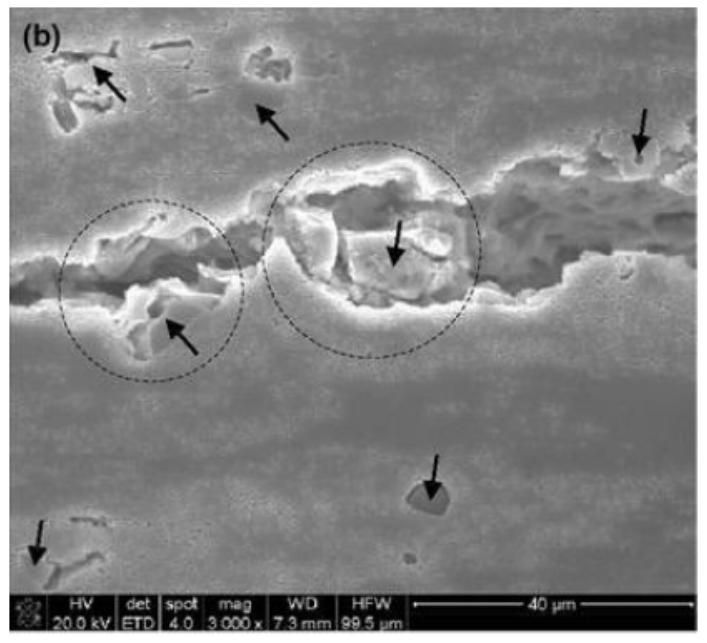

Figure 2.2: SEM imaging of armour grade aluminum post spall, showcasing debonding of the inter-metallic particles from the aluminium matrix [17]

as-received copper, which was attributed to the more comparable phase stiffnesses. From this study, it should also be noted that the aluminum-alumina $\left(\mathrm{Al}^{-} \mathrm{Al}_{2} \mathrm{O}_{3}\right)$ composite was observed to have a higher spall strength than other comparable aluminum-ceramic composites, a result attributed to a relatively strong interface adhesion between the aluminum and alumina [26].

Once again, considering the implications of favourable nucleation sites on spall strength, applied strain rate should be discussed. Here, conventional wisdoms dictates that a higher strain rate applies tensile loading across a narrower region $[11,13]$. With less material under tension, fewer nucleation sites would be available. Fewer favourable flaws under tension should in turn increase spall strength [11]. Higher spall strengths corresponding to an increase in strain rate have been seen for a number of metals including: Titanium alloy, Titanium, Stainless Steel, Copper, and Al-6\% Mg [11]. However, exceptions to all rules apply. For example, spall in Tantalum under varying strain rates saw little change in strength, along with a decrease in observed damage, with voids more spread out, and tending to be of a smaller size [13]. Likewise, in the case of armour steels (MARS 190, 240, and 300), increased strain rates saw reduced strengths, which in this case were attributed to 
a phase transition in the steel at higher impact velocities [14].

In addition to strain rate, wave profile will be discussed for its influence on spall strength. Wave profile can either exist as a square or triangular form [27]. Profile shape depends on the length of time between initial compression from the incident shock wave and the onset of tensile loading [28]. Waves tend to take on a square form, evolving into a triangular shape with the passage of time. This mechanism is discussed in further detail in Section 3.1. It has been shown that the onset of spalling in metals occurs at lower loading for square wave forms compared to triangular waves [28]. Studies investigating the influence of wave profiles on spall strength in 316 stainless steel found similar results [27]. Spall strength was found to have a strong dependence on wave profile. This effect was significant enough that square shaped loading at $6.6 \mathrm{GPa}$ initiated spall, while triangular loading at 10.2 GPa failed to display a tell-tale pullback velocity or associated spall damage. A tensile pressure of $15 \mathrm{GPa}$ was required to induce incipient spallation with a triangular wave profile [27].

\subsection{Spall in Polymers}

In the context of spall, polymers have been studied far less than metals [29]. Polymers will still be discussed in this Section, for their insight into failure mechanisms. For example, one study investigating spall in polycarbonate discusses crack coalescence in amorphous polymers, explaining the evolution of penny shaped cracks into dish shaped ones [30]. Further discussions regarding favourable flaw sites and strain rate effects can be seen in the spall of other neat polymers.

A study into the spall strength of silicon oils presented findings regarding the implications of "energy-limited" (heterogeneous) and "flaw-limited" (homogeneous) spall [31]. 
Spall in liquids results in the formation and growth of bubbles (cavitation), driven by the applied tensile load overcoming surface tension effects [31]. It was expected that the regime would change with an increase in strain rate (energy- to flaw-limited) due to fewer available favourable flaws present in the narrower tensile region. The authors found spall strength in the silicon oil was not influenced by the presence of pre-existing bubbles, but rather dominated by bubbles nucleated by voids formed "via random molecular fluctuations" [31]. This suggests these inherent voids served as sufficiently favourable flaws to induce spall, without the need for pre-existing bubbles.

A study on the effect of curing agent variations in epoxy observed a strain dependent spall strength, with the EPON 828 A epoxy showing a clear increase in spall strength with increasing strain rate [32]. The jump in spall strength at high strain rates was attributed to a restriction in molecular mobility, which inhibited deformation at the high strain rates observed in spall experiments. By varying the curing agents, the authors explained that it is likely the extent of cross-linking in the backbone polymer chains is modified, with the EPON 828 A having greater cross-linking. This reflects on molecular mobility and spall, with increased cross-linking likely to reduce molecular mobility.

Beyond the studies presented thus far, investigations of spall strength in polymers tend to focus primarily on epoxy composites $[19,21,33]$ reinforced with fibers or carbon nano tubes (CNT). An investigation of microstructure influences on CNT reinforced epoxy observed loading profile effects in the samples [19]. Use of an aluminium impactor resulted in unloading waves from the back face of the impactor overtaking and smearing out the incident wave, resulting in a triangular profile. As was the case with metals, this was observed by instrumentation as an immediate onset of unloading following the arrival of the shock wave. Experiments using an acrylic flyer, saw a clear delay between the shock wave arrival and onset of unloading [19]. Also noted was the fact that triangular loading resulted in a 
wider area of tensile loading due to the tensile region starting at the free surface and moving inward [19]. Due to the broader damaged region, it was observed that samples loaded with a triangular wave demonstrated a reduced spall pulse amplitude when compared to one resulting from a square wave. This reduced pulse amplitude was attributed to internal wave dispersion, due to it travelling through previously damaged material.

In this study, and others regarding fibre reinforced epoxies, there is evidence that the presence of nanotubes in an epoxy-CNT composite reduces the spall strength with respect to that of the neat epoxy $[18,19]$. Scanning electron microscopy (SEM) observed fibre pullout at the internal fracture surface (shown in Figure 2.3), which is indicative of a low strength interface between the CNTs and the epoxy interface. A low strength interface could increase nucleation density by behaving as the preferred location for void nucleation.

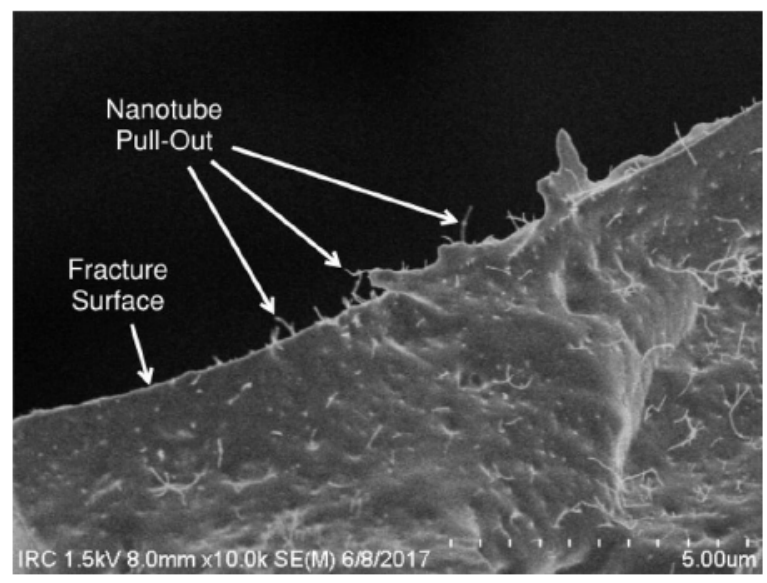

Figure 2.3: SEM imaging of epoxy-CNT composite post spall, showcasing nanotube pullout from the matrix $[18,19]$

However, in functionalized CNT-epoxy composites, it was found that an increased interface adhesion (from functionalization) negatively influenced spall strength when compared to an untreated sample, due a change the failure mechanism of the composite [33]. The dominant failure modes of CNT-epoxy composites have been described as: pullout of the 
fibre from the matrix (fibre pullout), fracture of the CNT (immediate fracture), and simultaneous pullout and fracture (sliding fracture) $[34,35]$. Sliding fraction is considered to be the best of the three mechanisms, as it delays fracture and dissipates energy as friction [33].

Similar observations have been seen in the use of larger fibers (micron scale) to reinforced the epoxy matrix [21]. A drastic reduction in spall strength of the functionalized composite (208 MPa $\pm 10 \mathrm{MPa}$ ) was observed when compared to the pristine fibre reinforced composite $(410 \mathrm{MPa} \pm 10 \mathrm{MPa})$. This contrasted starkly with the doubling of interfacial strength seen with functionalization of the fiber. Anisotropic thermal behaviour of the composite during layup was blamed for this behaviour; poor adhesion in the pristine fibers allowed the thermally-induced tensile state in the matrix to relax after manufacturing. It was proposed that strong matrix adhesion with the treated fibers would prevent this relaxation, inducing residual stresses, and reducing the overall spall strength [21].

In the quasi-static realm, a number of studies exist investigating the effect of filler treatment via silanes [36-39]. Functionalization in these polymer matrix composites tends to result in an increase in tensile strength and elastic modulus while decreasing the elongation to failure. Increased filler and/or silane content amplifies these effects $[36,38]$. These changes have been attributed to increased adhesion between the matrix and filler, improving stress-transfer from matrix to filler and limiting pullout of the filler from the matrix. Exceptions to these findings were seen in samples with poor filler dispersion $[36,37]$.

With the effect of inclusions and interface adhesion discussed here, particle morphology should be considered for the influence it has on the cumulative surface area of the matrixfiller interface. An increased interface surface area in a given composite may result in a higher density of nucleation zone candidates. Particle morphology can be numerically characterized using aspect ratio, which can be defined as the ratio between characteristic length and diameter of a particle. While spheres, with an aspect ratio of 1 occupy the middle of the aspect ratio spectrum, the ends of the spectrum are occupied by plates and 
rods [40], each possessing a very high surface area for a given volume. By extension, aspect ratio dictates a particle's surface area for a given volume. In terms of composite design this means two composites, filled with particles of the same size, at the same volume fraction, will have a different cumulative interface surface area if the filler aspect ratios differ.

Particle shape or aspect ratio has been observed to influence the quasi-static behaviour of particle-elastomer composites, with an irregular filler encouraging stiffer, higher strength response when compared to an otherwise identical spherical filler [41]. The increased performance was attributed to particles re-aligning under mechanical loading being better able to transfer the applied loads. In the ballistic realm, the irregular filler saw an increased performance compared to the spherical filler [41]. Irregular particles were believed to better resist lateral expansion due to stress concentration at the angular edges and improved strain hardening provided by the corners of the particles [41]. In the dynamic fracture regime, filler morphology in epoxy composites has been shown to influence fracture toughness. Filler with larger aspect ratios provided the largest increase in toughness when compared to the neat epoxy; $145 \%$ and $97 \%$ for rods and flakes respectively, compared to a $67 \%$ improvement for the spherical filler [42].

When reviewing polymers, it is also worth discussing elastomers, a class of polymers known for their "ability to undergo large reversible deformations" [11]. Due to this behaviour, their dynamic under spall is somewhat unique. Specifically, signals recovered from many elastomeric materials will appear quite different than commonly published results for metals [11]. Spall tests in rubbers have been observed to yield no distinct spall damage, even when the applied load exceeds the measured spall strength by a large margin. For this, the elastic behaviour of elastomers means that measured spall strengths likely denote the loads required to nucleate voids in the spall plane rather than the failure strength of the material $[11,43]$. Elastomers will also often show a brief pullback pulse rather than the more distinct pullback seen in most other materials. 
In conclusion, this chapter has presented a selection of pertinent literature regarding a range of materials, studied under a variety of loading conditions. Specific attention has been paid to the effect of favourable flaw sites on spall strength. In discussing these flaw sites it has been shown how conditions such as strain rate and loading profile, and properties such as matrix-filler adhesion and filler morphology influence material behaviour under dynamic tensile loading. Through the tailoring of material properties in this study, it is anticipated that the spall strength of the polymer matrix composites presented here can be modified. 


\section{Chapter 3}

\section{Wave Mechanics}

This chapter will briefly address the fundamentals of shock waves required for a proper appreciation of the wave mechanics involved in spall. It will begin with the formation of shock waves, touch upon wave interactions, and culminate with an explanation as to how spall failure is induced. The domain of shock waves is the realm of material compressibility. All matter is compressible, with perturbations transmitted as waves [44]. The analytical methods presented in this Chapter were relied upon to design the experiment and analyze the results presented later.

\subsection{Compressibility and Shock Waves}

This discussion begins with an elastic wave bound by a Control Volume (CV) as shown in Figure 3.1. Affixing the coordinate system to the wave provides a wave fixed frame of reference.

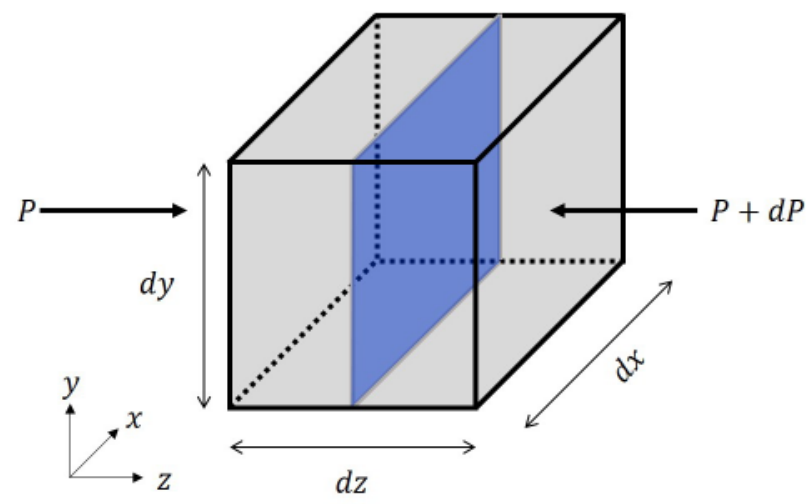

Figure 3.1: Control Volume Enclosing a Wave 
Applying Newton's second law across the control volume it can be seen that:

$$
\begin{aligned}
\Sigma F & =m a \\
(P-(P+d P)) *(d x * d y) & =\rho(d x * d y * d z) * \frac{d v}{d t} \\
d v & =\frac{-d P}{\rho} * \frac{d t}{d z} \\
d v & =\frac{-d P}{\rho} * \frac{1}{v}
\end{aligned}
$$

where $v$ is velocity, $\rho$ is density, and $P$ is pressure. Next, take conservation of mass across the CV:

$$
\begin{aligned}
\dot{m}_{\text {in }} & =\dot{m}_{\text {out }} \\
\rho * \text { Area } * v & =(\rho+d \rho) \operatorname{Area}(v+d v) \\
\rho * v & =\rho * v+\rho * d v+v * d \rho+d \rho * d v
\end{aligned}
$$

Taking the product of two infinitesimals $(d \rho * d v)$ to be zero (very small relative to the other terms) and simplifying yields:

$$
d v=\frac{-v * d \rho}{\rho}
$$

Lastly, combining Equations 3.1 and 3.2 gives the wave speed equation commonly seen:

$$
C^{2}=\frac{d P}{d \rho}
$$

where $C$ is the wave speed, $d P$ is the pressure differential across the wave and $\rho$ is density. This relationship shows that with an increasing pressure gradient comes increased wave velocity [44]. Showcased by Figure 3.2, the significance of this relationship can be seen in a wave where the pressure rises over time. Starting from the wave front and moving aft 
the pressure rises, driving an increased wave velocity $[8,44]$. An increasing wave velocity along the length of the wave will cause it to contract, culminating in the discontinuous form of a shock wave $[8,44]$.

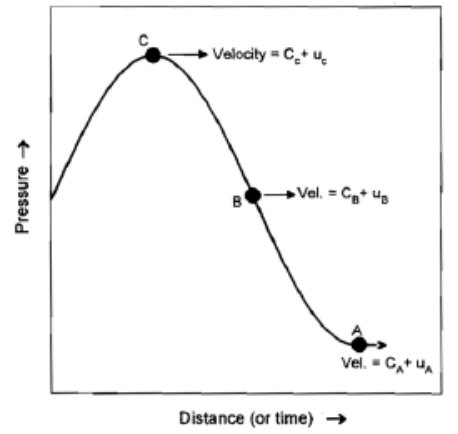

(a)

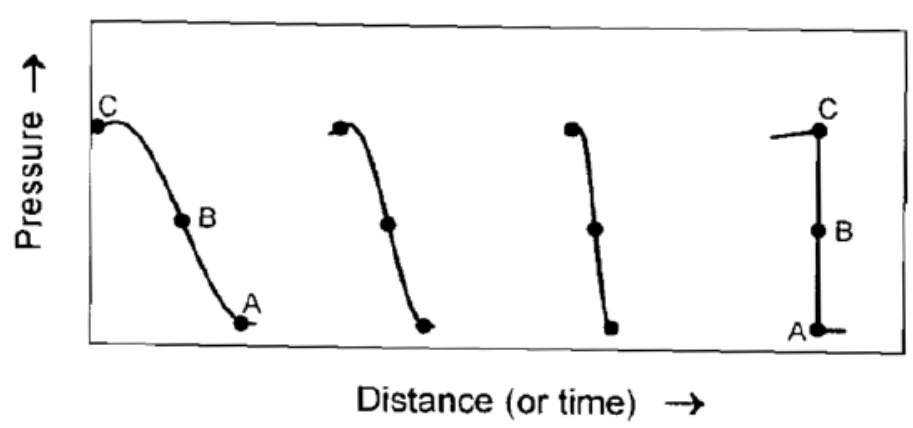

(b)

Figure 3.2: Representation of (a) the velocity along a wave and the (b) contraction of the wave length with the progression of time [8]

As shown here, pressure $(P)$ and density $(\rho)$ of the medium are used to define wave speed ( $C$ for sound waves, or $U_{s}$ for shock waves). Beyond these, particle velocity $\left(u_{p}\right)$, and internal energy $(e)$ are also required to constrain the state of the material subjected to the wave [8]. By knowing these five properties, one can effectively model the state of the material, before and after shock loading. Typically, the shock front fixed frame of reference [44], where the observer "rides" the moving shockwave is used [45]. From this, conservation of mass, momentum, and energy across the shockwave can be utilized to provide three of the needed relationships. These equations, known as the RankineHugoniot jump equations are shown below in Equations 3.4-3.6 respectively [8,45]:

$$
\begin{gathered}
\frac{\rho_{1}}{\rho_{0}}=\frac{U_{s}-u_{0}}{U_{s}-u_{1}}=\frac{v_{0}}{v_{1}} \\
P_{1}-P_{0}=\rho_{0}\left(u_{1}-u_{0}\right)\left(U_{s}-u_{0}\right)
\end{gathered}
$$




$$
e_{1}-e_{0}=\frac{P_{1} u_{1}-P_{0} u_{0}}{\rho_{0}\left(U_{s}-u_{0}\right)}-\frac{1}{2}\left(u_{1}^{2}-u_{0}^{2}\right)
$$

The 0 subscript denotes the state of material prior to shocking up, with the 1 denoting the state after. In many cases the pre-shocked state of the material is non-moving and unloaded, allowing for $P_{0}$ and $u_{0}$ to be set to zero, further simplifying these equations. Having defined three of the five relationships required, further relationships are required to constrain the system $[8,44,46]$. The material properties of the medium undergoing shock loading can provide these requisite relationships in the form of equations of state (EOS) [45]. In the case of gases, the ideal gas law is a simple EOS used to relate pressure, density, and temperature as function of the gas constant [8].

These relationships are called Hugoniots, and have been determined experimentally [45]. While any two of the five variables can be related, the $U_{s}-u_{p}, P-u_{p}$, and $P-v$ Hugoniots have proven to be the most useful [8]. Of particular interest to this study is the $P-u_{p}$ Hugoniot, which prescribes the particle velocity of the material as a function of the pressure of the shockwave. Algebraically, the $P-u_{p}$ Hugoniot takes the following form:

$$
P_{1}=\rho_{0} C_{0}\left(u_{1}-u_{0}\right)+\rho_{0} s\left(u_{1}-u_{0}\right)^{2}
$$

where $C_{0}$ and $s$ are experimentally determined values. The utility of this Hugoniot is its validity when the initial particle velocity is non-zero, as well as its ability to consider the directionality of the wave it is associated with. However, it is important to understand that a Hugoniot represents a series of end states, meaning that shocking a material causes a linear and direct jump in state from beginning to end state $[8,45]$. The material does not follow the path of the Hugoniot between two states.

Having discussed shockwaves and the associated change of state, the unloading of shock compressed matter must also be considered. In this case this is analogous to releasing a compressed sponge, it will unload and move to decompress itself [8]. Prior to 
discussing spall this mechanism of unloading must be understood. Unloading begins at an interface or free surface and is driven back as an infinite series of progressively slower wavelets into the previously compressed material. This series of wavelets (rarefaction fan) causes a gradual and isentropic unloading of the material [45]. As rarefaction fans travel through a material, the spacing between each individual wavelet increases, due to preceding wavelets unloading the medium and reducing density [8]. Furthermore, the leading edge of the rarefaction fan will overtake the shock wave it is pursuing, due to the rarefaction travelling in a medium compressed by the incident shock wave $[45,46]$. Figure 3.3 demonstrates both the rarefaction fan elongation and overtaking of preceding shockwave, showing how an initially square wave profile becomes triangular over time.

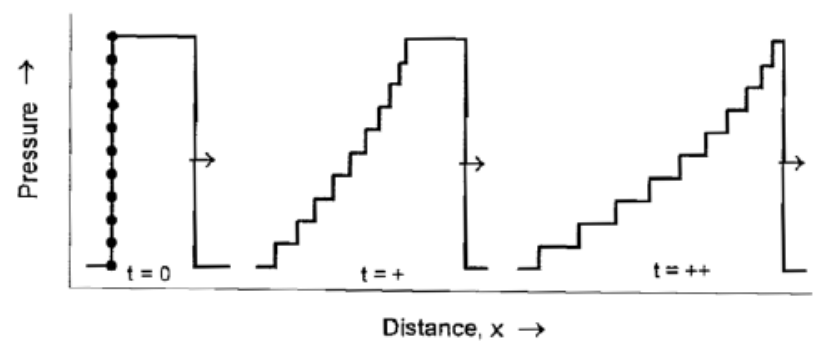

Figure 3.3: The separation of a rarefaction fan and its overtaking of a shockwave [8]

\subsection{Wave Interactions during a High Strain Rate Plate Impact}

Using the fundamentals discussed previously, this Section will address the wave interactions during a high strain plate impact. In this case a plate impact experiment consists of a face to face impact between a circular flat plate (flyer plate) and another circular plate (striker plate) at high speed. The material of interest (target) lies behind the striker plate and will be subjected to the wave generated by the initial impact as it travels through 
the striker plate and into the target. Any successful experiment relies on the generation of a uni-axial strain state in the target $[47,48]$, meaning that edge effects cannot influence the experiment. In a plate impact these edge effects exist as radially moving release waves generated by immediate unloading at the edges of the plates.. These edge release waves, denoted by $T$ and $T^{\prime}$ in Figure 3.4 move toward the centre of the plate invalidating the uni-axial strain condition.

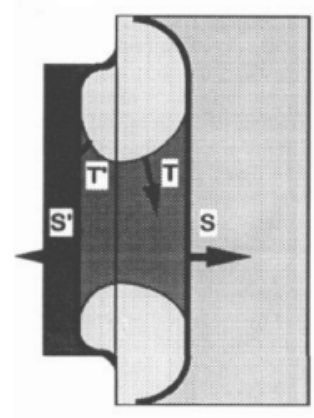

Figure 3.4: Wave structure of a plate impact experiment [49]

In order to maintain the requisite uni-axial loading condition required, spall and data collection must occur prior the arrival of these lateral waves. To avoid these edge effects a target diameter to target thickness ratio between 6 and 10 is typically used [47]. Assuming a complicit geometry, edge effects will be neglected in this discussion.

\subsubsection{Initial Impact}

Considering only the initial collision of the two plates, the impact can be considered an impact between two semi-infinite slabs, one moving $(A)$ and one stationary $(B)$, as shown in Figure 3.5. On impact, two shock waves will be generated at the collision interface, one left-going (LG) into $A$ and one right-going (RG) into $B$. Known are the initial states of both slabs, specifically $\rho_{0}, u_{0}$, and $P_{0}$ as well as the Hugoniot properties of both slabs. Due to the laws of conservation it is also known that the pressure and particle velocity of the matter 
between the two shock fronts will be equal [45].

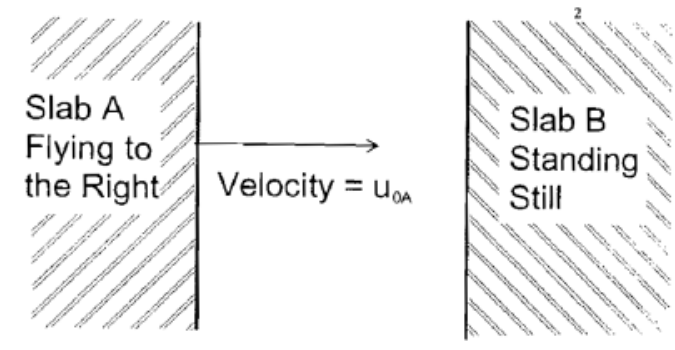

Figure 3.5: Schematic depicting the impact of two semi-infinite slabs [8]

The $P-u_{p}$ Hugoniot is used as it is able to consider the non-zero initial velocity of $A$ and the directionality of the generated shock waves. Applying the shock properties of the two slabs, the Hugoniots of both shock waves are plotted, as shown in Figure 3.6. The blue, right-going Hugoniot originates from $P=0$ and $u_{p}=0$ to represent the initial conditions of $B$, with the RG nature of the Hugoniot reflecting the directionality of the RG wave generated in $B$. Likewise, $A$ is represented by the red, LG Hugoniot, which depicts the LG wave generated in slab $A$ and its in-motion, unloaded initial state. From the intersection of the two Hugoniots in Fig 3.6 particle velocity and pressure of the "Shocked State" are found. Likewise, shock velocity can be determined using the momentum equation.

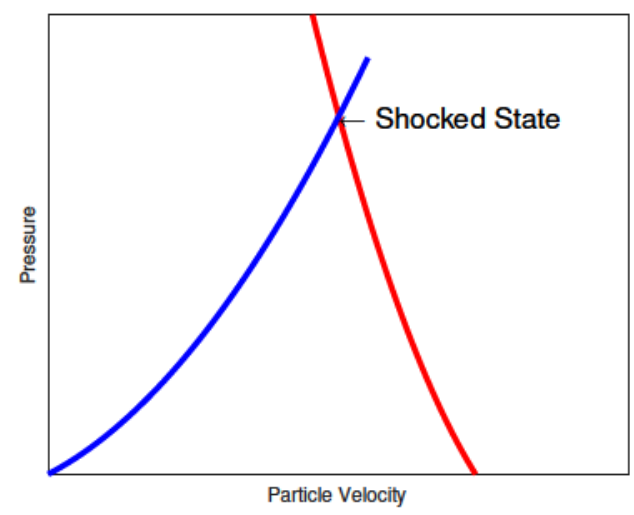

Figure 3.6: $P-u_{p}$ Hugoniot depicting an initial impact between two materials 


\subsubsection{Transmission of Waves between Mediums}

In the case where the impact is between two plates of a finite thickness, the consequences of the shock wave travelling the length of the striker plate $(B)$ and entering a new medium $(C)$ should be considered. Once again, the Hugoniots of the two mediums can be used to model the interaction. Known initial conditions are: the initial state of $C$ (at rest), the Hugoniots of both mediums, and the previously determined shocked state of $B$. Furthermore the transition of $B$ from the "Initial Impact State" to the "New State" will follow its Hugoniot [8].

Like before, the $P-u_{p}$ Hugoniot is used to model the transition of a shockwave. However, in this case the Hugoniot diagram begins from the Initial Impact State and transitions to a new one. Known is that the LG Hugoniot of material $B$ (solid blue line) crosses the initial impact state, and that the RG Hugoniot of material $C$ (solid black line) originates from $u_{p}=0, P=0$, as these points depict the material states prior to arrival of the shockwave. Drawing the Hugoniots with these details provides one meeting point, which denotes the new state as shown in Figure 3.7.

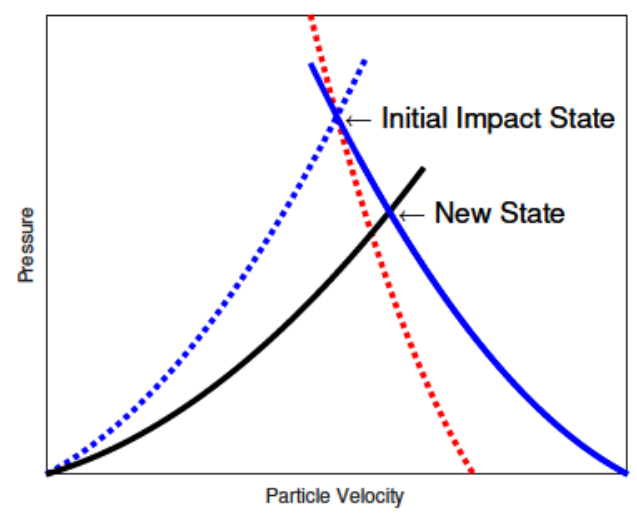

Figure 3.7: $P-u_{p}$ Hugoniot depicting the arrival of a wave at an interface between two materials

It is worth noting that in the case shown here the reduction of pressure between the 
"Initial Impact State" and "New State" is actualized as a LG rarefaction fan travelling back into $B$, reducing the pressure in $B$ from $P_{\text {Initial Impact State }}$ to $P_{\text {New State }}$. This is a consequence of relative impedance between the two materials. Impedance $(Z)$ can be approximated as the product of density and sound speed $\left(Z=\rho_{0} * C_{0}\right)$. Relative impedances dictate the reflected and transmitted wave type (shock vs. rarefaction) and their magnitude, with larger differences driving larger magnitude reflections [45].

\subsubsection{Arrival of a Wave at a Free Surface}

Once again, considering finite thickness, the arrival of the wave at the far side of the target will be addressed. Upon reaching a free surface the wave is now attempting to travel through air/vacuum, which encourages the highly compressed material at the free surface to unload and accelerate. From the "New State", the matter at the free surface will unload due to the generation of a LG rarefaction fan, back into the target. This LG rarefaction fan is depicted in Figure 3.8 by the solid black LG Hugoniot joining the "New State" and "Unloaded State".

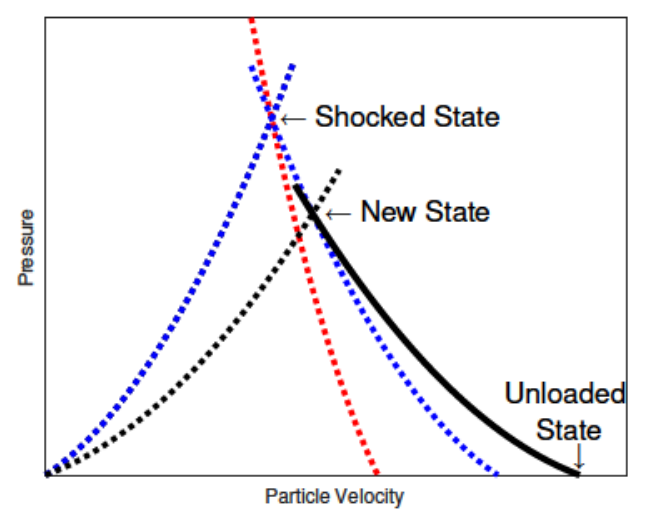

Figure 3.8: $P-u_{p}$ Hugoniot depicting the unloading of a material via the arrival of a shock wave at a free surface 
Upon review of the $P-u_{p}$ Hugoniot of a material unloading one will see that the material will essentially trade energy stored as pressure for increased particle velocity, specifically one that is twice that of the particle velocity of the shocked state. This proves useful as observation of the free surface velocity of the material provides the particle velocity associated with the shocked state of the material [8].

Lastly, if rarefaction fans are a consequence of a shock wave arriving at a free surface, the implications of the two incident shock waves (left- and right-going) generated in this impact arriving at the free surfaces of the impactor and target respectively must be considered. When both opposing free surfaces generate rarefaction fans travelling back into the material there will be repercussions from the interactions of these two rarefaction fans. Under sufficient loading the consequence is spall, a phenomenon discussed in detail in the following Section.

\subsection{Spall Mechanics}

To those otherwise unfamiliar with the concept of spall in a shock physics context, it can be considered comparable to Newton's cradle. Newton's cradle is a tabletop toy shown in Figure 3.9 (a) that demonstrates elastic collisions when a moving ball bearing collides with a row of identical stationary ball bearings; the end result being the one ball opposite of the striking side is ejected. "Spallation" is demonstrated by the ejection of the right most bearing, a consequence of the impact of the left most bearing.

Figure 3.9 (b) shows an x-t diagram depicting the wave behaviour over time. An x-t diagram, also known as a distance-time plot is used to graphically represent the travel of waves along one axis as a function of time. Here the $\mathrm{x}$-axis is considered as a horizontal line travelling through the line of bearings, as seen in Figure 3.9 (b). The origin of an $x-t$ plot depicts the location and time of the initial impact, in this case the instant of impact 


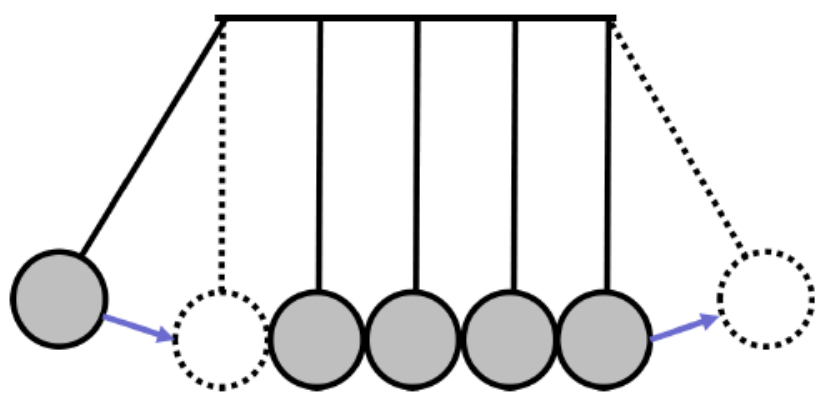

(a)

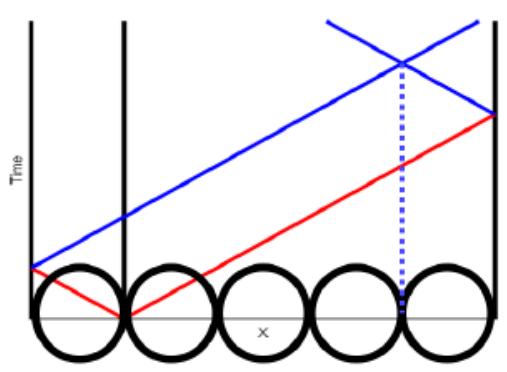

(b)

Figure 3.9: (a) Schematic and (b) $x-t$ diagram of Newton's cradle in operation.

between the first and second bearing. Here, elastic waves (red) are generated, and travel into the bearings. After some time the waves reach the left- and right-hand free surfaces and are reflected as unloading waves (blue). The intersection of these unloading waves corresponds to the separation point formed between the fourth and fifth ball.

Spall in plate impact experiments, as depicted in Figure 3.10 is a similar event where the high velocity collision of an impactor plate into a target results in the ejection of target material from the face opposite of the impact surface [8]. Experimentally, spall can be induced by a range of apparatuses and loading conditions such as laser ablation $[11,50,51]$ or ballistic impacts [45]. In the context of the study presented here, only spall resulting from plate impact experiments will be considered.

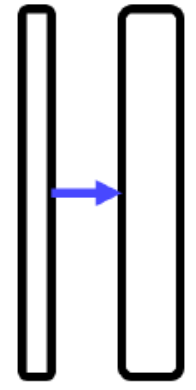

(a)

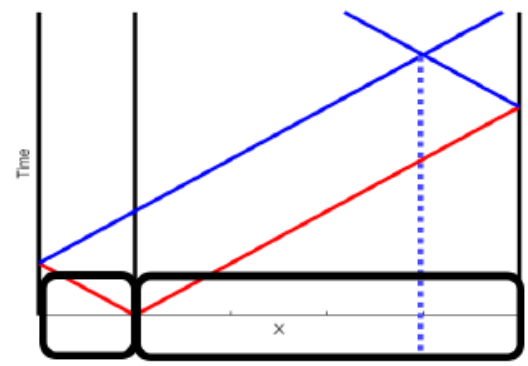

(b)

Figure 3.10: (a) Schematic and (b) $x-t$ diagram of spall in a flat plate impact. 
Spall is caused by the interaction of two unloading waves colliding head on, causing constructive interference [45]. Rather than only releasing the compression in the shocked material, the interference drives the material from a compressed state to zero compression, and on to a state of tension along the plane where the two rarefactions fans meet (called the spall plane) [8]. If the resultant tensile loading from the interaction of the rarefaction fans exceeds the spall strength of the loaded material, spall will occur, causing internal tensile failure. Spall results in the generation of an internal fracture surface. Shown in Figure 3.11 is an $\mathrm{x}$-t diagram depicting the wave interactions resulting in the interaction of the rarefaction fans, responsible for the formation of the spall plane.

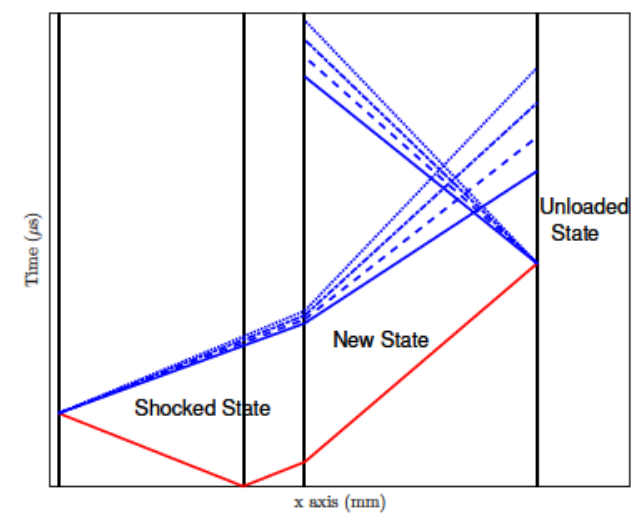

(a)

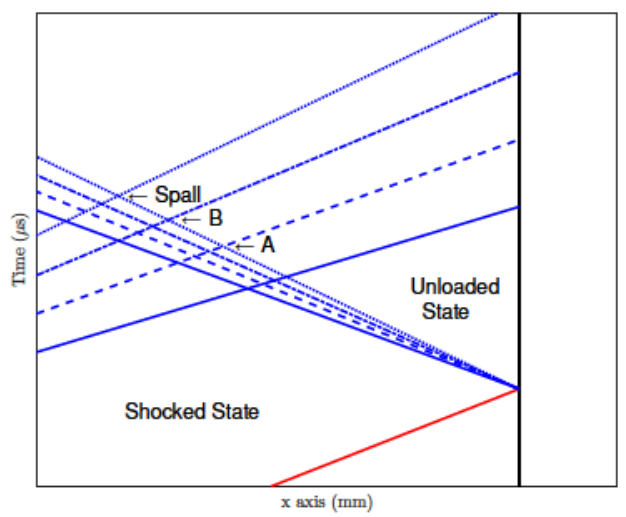

(b)

Figure 3.11: a) $x-t$ diagram showing generation of spall plane in a target (b) same $x-t$ diagram as in (a) zoomed to region where the rarefaction fans overlap

To better represent the wave interactions that result in spall, the effective depiction of spall in the $P-u_{p}$ Hugoniot (as shown in Figure 3.12) should be discussed. With a pair of rarefaction fans generated from the opposing flyer plate and target free surfaces travelling towards each other, a tensile state will be induced where the rarefactions meet. With everything still moving in the positive direction, the points representing the tensile state developing within the target thus lie in the positive velocity, negative pressure region. 
It is also known that Hugoniots in the negative pressure region can be approximated by linear functions with a slope of $\rho_{0} * c_{0}[8]$.

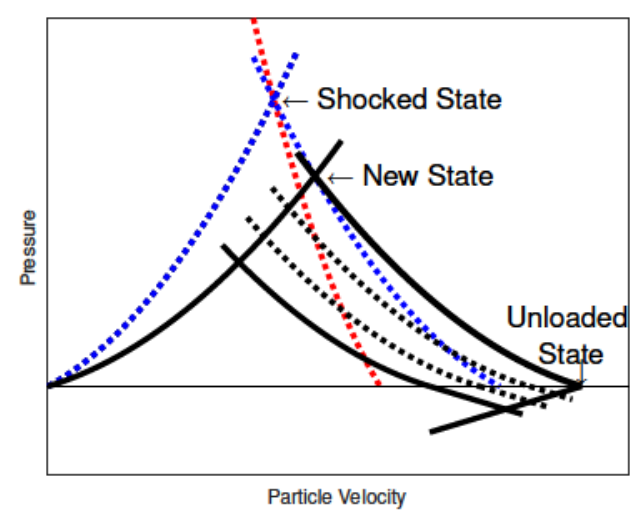

(a)

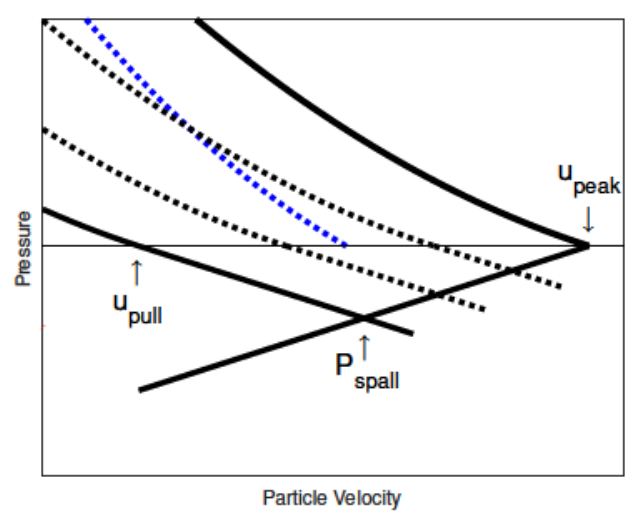

(b)

Figure 3.12: (a) $P-u_{p}$ Hugoniot depicting the development of tension during spallation of a target (b) Same Hugoniot as in (a) zoomed to focus on the negative pressure region

Next to consider is the known states. The state of the LG rarefaction is the fully unloaded state. The RG rarefaction fan is represented by the RG Hugoniot going between $u_{\text {peak }}$ and $P_{\text {spall }}$ in Figure 3.12 (b). It should be remembered that each wavelet can be represented by a short step along the RG Hugoniot of the rarefaction fan as seen in Figure 3.12 (a). As each RG wavelet crosses paths with the $\mathrm{LG}$ fan, the resultant state must be determined. Drawing LG Hugoniots from these wavelet states, the induced tensile state is revealed and depicted. As each successive RG wavelet crosses the length of the LG fan, the increasing tensile state develops within the material. When the resulting tensile loads exceed the strength of the material a spall plane is generated. 


\subsection{Spall Strength}

With spall being a high strain rate event occurring within a typically opaque material, one must be able to infer the spall strength of a material during a high velocity impact. Contemporary spall research relies on the fact that the particle velocity within a shocked material can be related to the free surface velocity of the target. Observing the velocity of the target free surface during spall tends to show a few distinct phases, as can be seen in Figure 3.13. The three phases of interest are the arrival of the shockwave at the free surface, unloading, and the pullback. Of particular interest is the peak velocity resulting from the arrival of the shock wave, and the minimum velocity seen after as they can be used to determine spall strength.

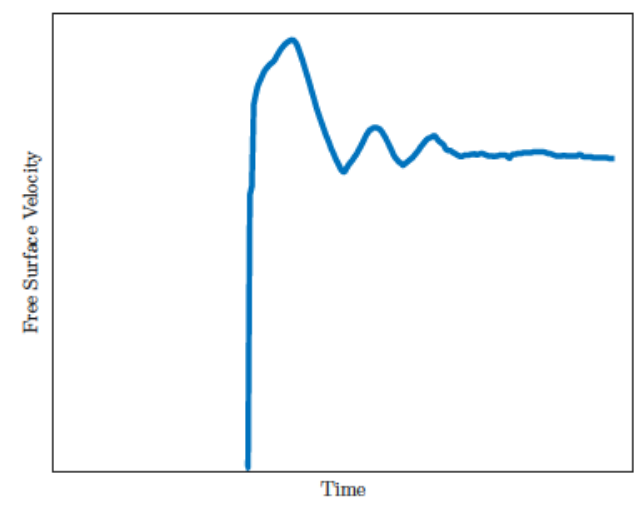

Figure 3.13: General back face velocity schematic during spall in neat polyurethane

A straightforward spall model was developed by Novikov et al. [52], which uses an acoustic approach to relate spall strength $\left(\sigma_{\text {spall }}\right)$ to the pullback velocity. This model determines spall strength as the intersection point of acoustic trajectories $\left(P_{\text {spall }}\right)$ on the $P-u_{p}$ plane as was depicted in Figure $3.12[11,53,54]$. Once again, the trajectories in the negative pressure region of the Hugoniot are defined by $\rho_{0} * c_{0}$ [8]. This intersection point also lies at the intersection of the two acoustic trajectories originating from the peak and 
pullback velocities. Extracting the stress state from the peak and pullback velocities is a function of slope and the velocity differences allowing spall strength to be defined as:

$$
P=-\rho_{0} c_{0} * \frac{\left(u_{\text {peak }}-u_{\text {pull }}\right)}{2}
$$

This equation, when simplified gives the following form, widely seen in literature [11]:

$$
\sigma_{\text {spall }}=\frac{1}{2} \rho_{0} C_{0} \Delta u_{f s}
$$

where $\sigma_{\text {spall }}$ is spall strength, $\Delta u_{f s}$ is the difference between $u_{\text {peak }}$ and $u_{\text {pull }}, \rho_{0}$ is density, and $C_{0}$ is bulk sound speed. Equation 3.9 shows that experimentally determined spall strength is a function of free surface velocity, density, and bulk sound speed. The determination of $\Delta u_{f s}$ has been discussed, but density and sound speed have not. Density is simple and measurable with readily available lab equipment. Sound speed is more complicated and will be discussed next in Section 3.4.1.

\subsubsection{Acoustics and Composites}

First, while $C_{0}$ is called the bulk sound speed it is not directly a material property, but rather the y-intercept of the $U_{s}-u_{p}$ Hugoniot [8]. Sound speed will be considered from a theoretical point of view. Sound speed in a material is a function of a material's density and stiffness as can be seen in Equation 3.10:

$$
C=\sqrt{\frac{E}{\rho}}
$$

where $E$ is Young's Modulus and $\rho$ is density. Determination of density has been discussed. Young's Modulus in a composite is less straightforward, and models have been developed to approximate it. Three models are commonly used for estimating the elastic modulus 
of two phase composites [55] as a function of volume fraction and phase stiffness. The Reuss model assumes constant stress meaning an equal stress state lies between the two phases of the composite. The Voigt model assumes constant strain between the phases, which typically applies to fiber reinforced composites with their fibers oriented parallel to the applied load. Lastly, the logarithmic model predicts an intermediate stiffness between the first two models [55].

$$
\begin{gathered}
E_{\text {Reuss }}=\frac{E_{1} * E_{2}}{V F_{1} * E_{2}+V F_{2} * E_{1}} \\
E_{\text {Voigt }}=V F_{1} * E_{1}+V F_{2} * E_{2} \\
\ln \left(E_{\text {log }}\right)=V F_{1} * \ln \left(E_{1}\right)+V F_{2} * \ln \left(E_{2}\right)
\end{gathered}
$$

Past work investigating particle reinforced composites has found that sound speed estimates using the Reuss model are the most accurate $[56,57]$. Since density plays a role in the sound speed of a material, at low volume fractions, little increase in overall sound speed may be observed in a composite, due to a linear increase in density, paired with a non-linear increase in stiffness. In fact, a decrease in sound speed is commonly seen at these low volume fractions $[56,57]$.

Moving back to bulk sound speed, $C_{0}$ has been related to the longitudinal $\left(C_{L}\right)$ and shear sound speeds $\left(C_{S}\right)$ of a material $[44,58]$. More specifically, they have been related by the function shown in Equation 3.14. This is useful as it allows for determination of $C_{0}$ directly using ultra-sonic (pulse-echo transit) measurements, rather than extrapolating $C_{0}$ from high strain rate experimental data. Sound speed was considered from a theoretical perspective to provide a benchmark against which the measured values can be compared.

$$
C_{0}=\sqrt{C_{L}^{2}-\frac{4}{3} C_{s}^{2}}
$$


Beyond sound speed, attenuation of an acoustic wave in a polymer particle composites should be considered as drastic attenuation has been seen in polymer particle composites, especially in rubbers [59]. An increase in attenuation is seen with an increase in ultrasonic wave frequency. Also of concern is the idea of acoustic scattering, whereby the wave disperses in a heterogeneous material due to a change in medium. It is also interesting to note that attenuation in a composite has been attributed to poor interface adhesion [60]. These effects were kept in mind when comparing the measured and theoretical sound speeds. 


\section{Chapter 4}

\section{Experimental Details}

The topics to be discussed in this chapter include sample preparation, experimental methodology, instrumentation, and data processing. Initially, the experiments used DOW Corning Sylgard 184, a vinyl-terminated polydimethylsiloxane (PDMS) and micron-scale, spherical aluminum oxide. The alumina powder integrated into the composite was either left untreated, treated for increased adhesion, or treated to impede adhesion. While the PDMS provided useful insight into the effect of surface adhesion, its low strength made observing the influence of surface adhesion more difficult than anticipated. A flexible, thermoset polyurethane (PU) was selected as a matrix material for the subsequent study, due to its superior mechanical properties while maintaining comparable ease of use, with respect to the Sylgard 184.

In addition, the PU study used a micron-scale, irregularly shaped alumina powder alongside the aforementioned spherical powder to observe the effects of particle morphology on spall strength. Beyond the change in polymer matrix material, the experimental configurations of both studies differed somewhat, through variations in target thickness, instrumentation, flyer plate thickness/material, and striking velocity. As such, the experimental details of the PDMS study has been segregated into Section 4.4 to provide a clear depiction of both experimental set ups. 


\subsection{Sample Preparation}

Preparation of the samples used in this study relied on a multi-step, multi-day process, requiring drying of the filler powder, functionalization of the particle surfaces with silane, and finally integration of the powder into the polymer matrix. Both alumina powders used in this study were sourced from Panadyne Abrasives. Prior to integration, the alumina particles shown in Figure tank4.1 were dried in a vacuum oven at $180^{\circ} \mathrm{C}$ for twelve hours to remove moisture, allowed to cool, then stored in a nitrogen purged container to maintain a desicated state.

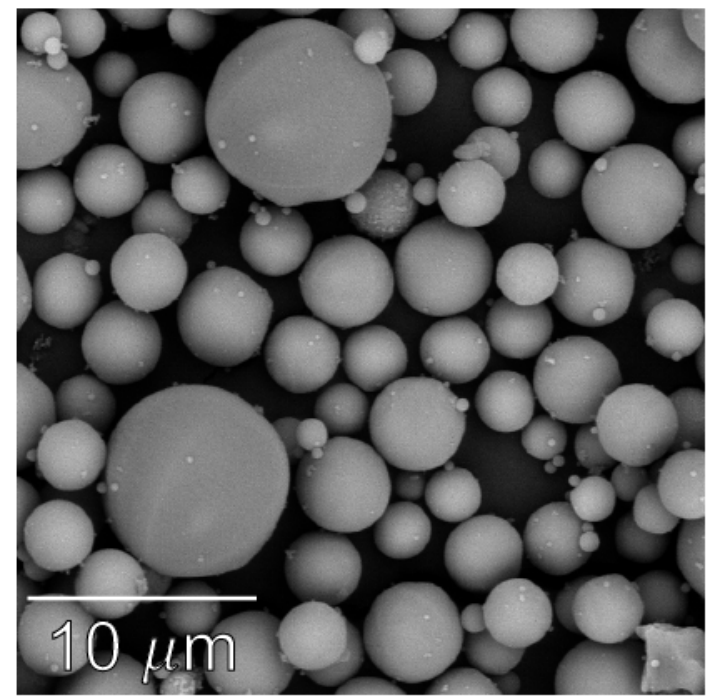

(a)

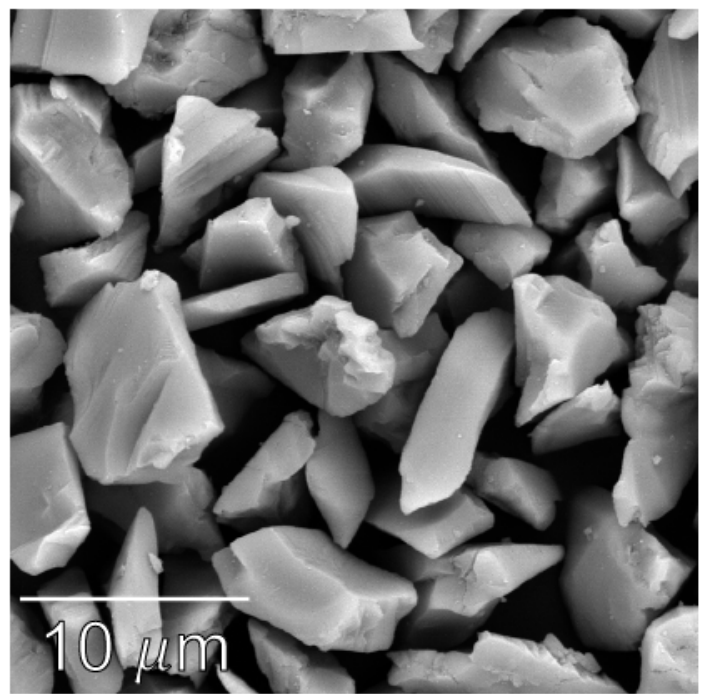

(b)

Figure 4.1: SEM images of the (a) five $\mu \mathrm{m}$ spherical alumina [41] and (b) 1000 grit irregular alumina [20] powders used in this study

With the information provided by Panadyne, Table 4.1 lists the size distribution of the alumina particles used in this study. 


\begin{tabular}{|c|c|c|c|}
\hline Filler material & $\mathrm{D}_{3}(\mu \mathrm{m})$ & $\mathrm{D}_{50}(\mu \mathrm{m})$ & $\mathrm{D}_{94}(\mu \mathrm{m})$ \\
\hline Spherical Alumina & 10.98 & 5.34 & 2.89 \\
Irregular Alumina & 6.95 & 4.58 & 2.98 \\
\hline
\end{tabular}

Table 4.1: Particle size distributions of the alumina filler used in this study

\subsubsection{Filler Treatment with Silane}

Interface adhesion between the polymer and ceramic phases was tailored using two silanes obtained from Gelest Inc. In a topical sense silanes are to ceramics and polymers, as soaps are to polar (water) and non-polar (oils) chemicals. With its long molecule, soap acts as a chemical intermediary, allowing water molecules to bond with oil molecules via the soap molecule. This bond allows for the removal of oils from dishes and textiles through mechanical forces in the washing process. Silanes, like soap rely on a long molecule with two unique ends to facilitate bonding to the unique surfaces present on either end of the molecule, in this case ceramics and polymers.

Silanes were selected in consultation with chemists from Gelest Inc. The first silane, Methacryloxypropyltriethoxysilane (Gelest trade-name SIM6487.4), was chosen to improve the adhesion between the polymer and alumina, while, n-octyltriethoxysilane (Gelest trade-name SIO6715.0), was chosen to suppress or impede adhesion. Schematics of these silanes are shown in Figure 4.2. A silane mass fraction of 5\% (with respect to the alumina filler) was selected based on literature survey of silane treatments for filler materials $[36,38]$.

To coat the dried alumina particles, five grams of the appropriate liquid silane was diluted in 10 grams of ethanol, then added to 100 grams of alumina. An additional 40 grams of ethanol was added to the mixture and stirred by hand until the alumina particles were well suspended in the solution. An ultra-sonic probe mixer (Fisher Scientific FB 505) was used to thoroughly mix the solution at $70 \%$ amplitude. A pulse cycle of 20 seconds 


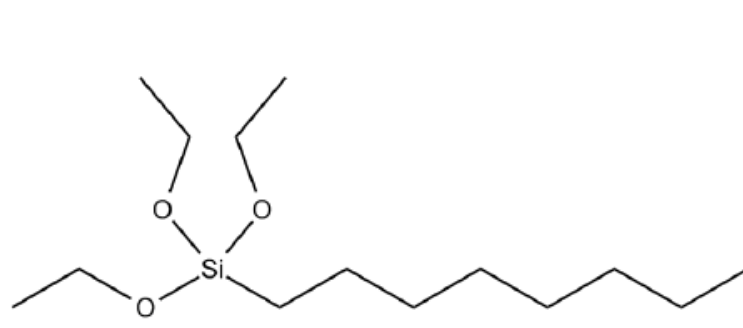

(a)

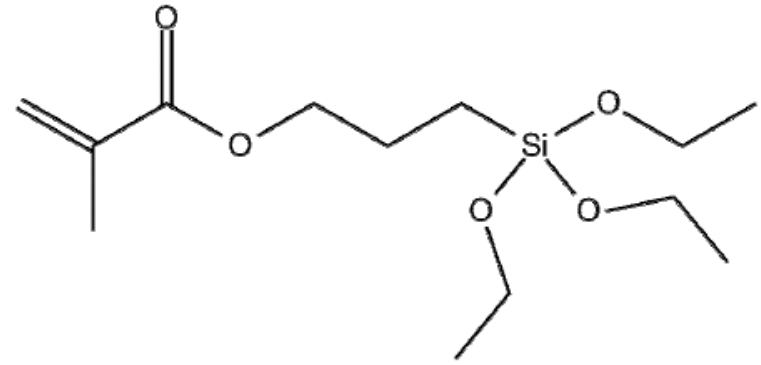

(b)

Figure 4.2: Schematics depicting the (a) aliphatic terminated (SIO6715.0) and (b) acrylate terminated (SIM6487.3) silane molecules used in this study [61]

on: 10 seconds off was used, for an elapsed mixing time of 5 minutes. The mixture was then placed in an oven at $60^{\circ} \mathrm{C}$ oven overnight, in order to evaporate the ethanol. The same procedure was used for both silane treatments.

\subsubsection{Preparation of Polyurethane Samples}

ClearFlex 95 (Smooth On) polyurethane (PU) was selected because of its past use within the research group, and ease of use. A $20 \%$ filler volume fraction was selected for the PU, as it was found to be the highest usable volume fraction that could be consistently manufactured to a satisfactory degree of quality. Higher volume fractions were found to be difficult to mix and de-gas. Only pristine alumina and alumina treated for impeded adhesion were integrated into the PU composites.

The samples were cast directly onto $1.6 \mathrm{~mm}(1 / 16 ")$ thick acrylic striker plates for a combined thickness of $\sim 8.5 \mathrm{~mm}$ thick. The acrylic striker plate was integrated to ensure a flat striking surface and inhibit bowing of the target during experiments. The samples integrating silane-treated alumina differed somewhat, with all but one being cast without an acrylic striker plate, to a thickness of $\sim 5 \mathrm{~mm}$. This was done to mitigate wave attenuation in the thicker samples, which was interfering with data collection. A pair of neat 
PU samples were also cast at this $5 \mathrm{~mm}$ thickness to provide a direct comparison between sample types cast at this reduced thickness.

The back surface of the samples were found to very smooth, possessing a clean, high gloss finish. As described by Smooth On in its manufacturing instructions, Ease Release 200 (Smooth On) mould release spray was applied to all surfaces of the mould, as well as the base of the acrylic plate (facing the mould base), to facilitate removal of the finished sample. Care was taken to avoid getting mould release on the top surface of the striker plate, as this would interfere with the bond between the PU and acrylic.

Alumina was added at a $20 \%$ volume fraction to each part (A and B) of the PU resin and mixed with a magnetic stirrer for five minutes, followed by sonication for 5 minutes at a 70\% amplitude. After being allowed to cool parts A and B were combined and mixed via magnetic stirrer for four minutes. Care was taken to inhibit the formation of a vortex, as the vortex was found to entrain large quantities of air in the PU. The mix was poured into open-faced moulds and degassed for four minutes. Any remaining bubbles or foam were manually popped or scraped off, prior to curing. Next, the moulds were covered with clean, glass dishware to inhibit any contamination of the open face moulds by dust. Samples were then cured at room-temperature for 6 hours, prior to post curing in an oven, at $72^{\circ} \mathrm{C}$ for 16 hours. After being allowed to cool, samples were removed from their moulds. Prior to any use or testing, a waiting period of at least 48 hours was observed to eliminate concerns of short term ageing effects.

\subsubsection{Preparation of PDMS Samples}

The other composite used in the present study comprised of an elastomeric polymer (Sylgard 184) and a ceramic filler particle (alumina). DOW Corning Sylgard 184 is a vinyl terminated polydimethylsiloxane (PDMS) that was selected for use in the present study due to its relatively low cost, ease of use, and the availability of published data in the 
literature [62-64]. The ceramic filler consisted of spherical alumina particles (Figure 4.1) with a mean diameter of five microns and were sourced from Panadyne Inc. The particles were used to reinforce the PDMS at a 33\% volume fraction, which was consistent for all of the samples prepared. Three sample materials were prepared, one in which no treatment was applied to the alumina, one in which the adhesion of the particles was increased, and another for which it was suppressed.

Spall samples were manufactured at a 33\% filler volume fraction. Alumina was added to the PDMS base and stirred by hand to wet the alumina. The mixture was subsequently placed in a high shear mixer to break up major agglomerations prior to sonication (same settings as listed above). After allowing the mixture to cool following sonication the curing agent was added to the mixture with a 10:1 base to curing agent ratio by weight. The mixture was then degassed under vacuum for one hour, poured into open faced moulds and degassed for an additional hour. The samples were cured in an oven at $120^{\circ} \mathrm{C}$ for 1 hour, allowed to cool, and removed from their moulds.

A density of $2 \mathrm{~g} / \mathrm{cm}^{3}$ was predicted for the PDMS samples. Sound speed of the PDMS samples was estimated to be $2.42 \mathrm{~km} / \mathrm{s}$ using a simple mixture model shown below in Equation 4.1:

$$
C_{0}=\left(\frac{\chi_{\text {filler }}}{C_{0 \text { filler }}}+\frac{1-\chi_{\text {filler }}}{C_{0 \text { matrix }}}\right)^{-1}
$$

where $\chi$ is the mass fraction of the filler.

\subsection{Single Stage Light Gas Gun}

A Single Stage Light Gas Gun (SSLGG) is an experimental apparatus common in experimental high strain rate impacts and shock propagation research. At its core the SSLGG is an apparatus consisting of three main sections; a high pressure breech section used to 
hold the driving gas, a barrel for accelerating the projectile, and a catch tank, which holds the experiment and contains the ejected gases, projectile fragments and target. Figure 4.3 shows a schematic of these elements of the gas gun. The SSLGG at Carleton University has a peak driving velocity of $\sim 750 \mathrm{~m} / \mathrm{s}$ when using helium as the driving gas. Readers interested in the process and mathematics behind gas gun design are directed to the "Theory of High Speed Guns" [65] written by Arnold Seigel.

The barrel section of the SSLGG consists of a tubular section with an internal diameter of $67 \mathrm{~mm}(2.645 ")$ and a length of $5 \mathrm{~m}$. Mounted to the end of the gun is a set of induction coil gauges for determining projectile velocity (discussed in detail later in Section 4.6.1). The barrel and breech sections are mounted on roller assemblies to facilitate loading of the gun and allow the gun to safely recoil in a direction co-linear with the axis of the gun.

Lastly is the catch tank section, which houses the target and is evacuated to $150 P a_{a b s}$ or below, prior to firing. The catch tank was deliberately designed to be large enough to ensure the entire system remains under vacuum after firing; inhibiting the opening of a pressurized system that would pose a safety hazard to operators. Projectiles are stopped within the catch tank using a sacrificial backing consisting of a rubber block and aluminum anvil. This protects the catch tank access flange, directly downrange of the muzzle.

Firing of the gas gun is controlled through the use of Mylar films in the breech, which segregates the high pressure region (HP) and intermediate pressure (IP) region, as well as segregating the IP region from the evacuated barrel/catch tank. The Mylar films are taped in place between the flanges of the breech section. The benefit of this dual diaphragm design is control of the driving pressure and the moment of firing. Rather than filling the HP region to the burst pressure of a single diaphragm, firing occurs by equalization of the HP and IP regions; the small volume ratio of the IP region to the HP region brings the pressure of the IP region up to that of the HP region. Equalization almost immediately bursts the 


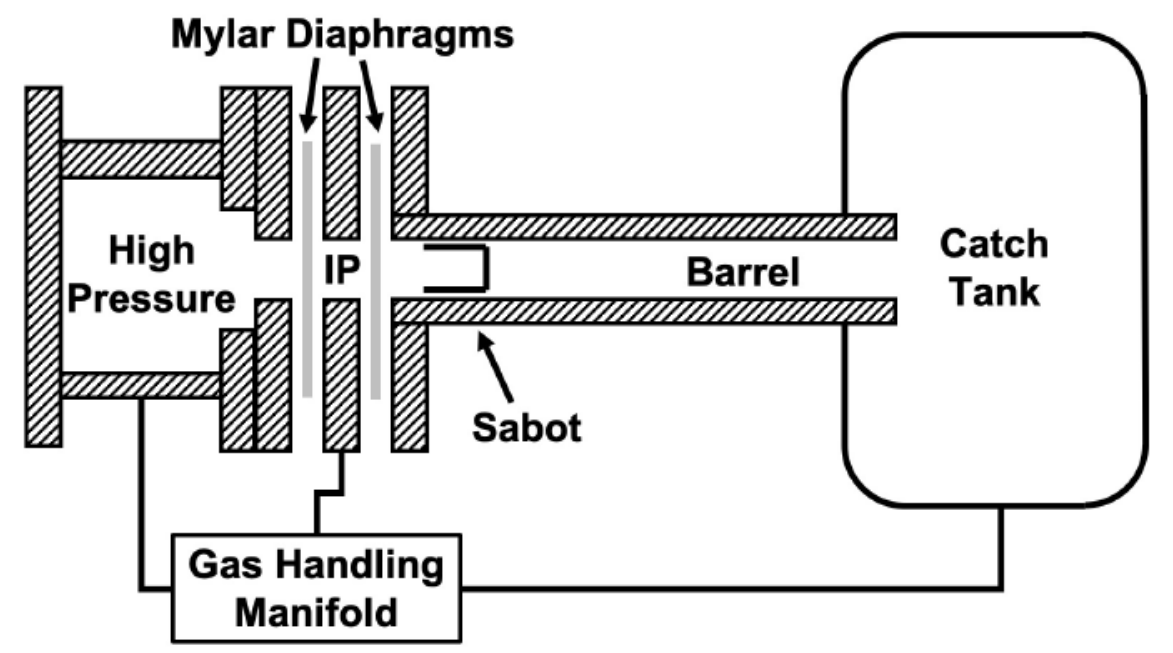

Figure 4.3: Schematic of the gas gun, showing the breech, barrel, and catch tank regions

diaphragms, due to the excessive pressure gradient now existing across the diaphragms.

\subsection{Plate Impact Testing}

The samples were subjected to a thin flyer plate impact to induce an internal dynamic tensile loading condition. The flyer plate was launched using the aforementioned $67 \mathrm{~mm}$ (2.645") internal diameter smooth-bore single-stage gas gun. Targets were instrumented with a two-channel photonic Doppler velocimeter (PDV) [66] to provide redundancy in data acquisition. An aluminized Mylar foil adhered to the back face of the sample with cyanoacrylate served as a reflector for the two PDV laser collimators, allowing for observation of back face velocity. A schematic depicting a mounted and instrumented sample is shown in Figure 4.4.

The instrumented samples were mounted in a machined target assembly affixed to the end of the barrel with a small offset to ensure planarity of the mounted target with respect to the flyer plate. Planarity is defined as the degree of tilt between the target striking surface and the flyer plate, typically measured in milli-radians. A magnet mounted within the sabot 


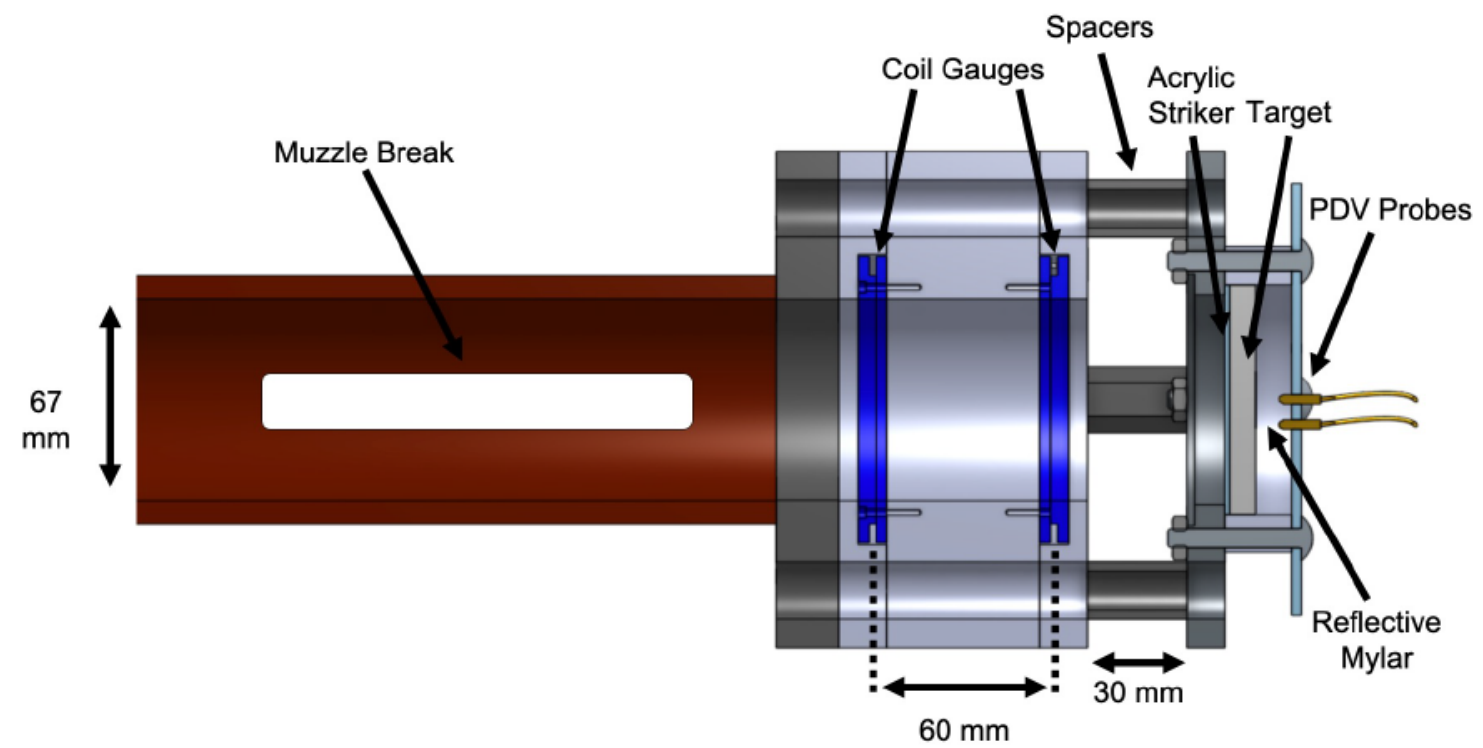

Figure 4.4: Schematic of instrumented sample mounted to the coil gauges on the muzzle of the gas gun

was used in conjunction with a pair of annular coil gauges to determine flyer velocity at impact based on transit times. A $89 \mathrm{~mm}$ (3.5") long PVC sabot supported the $64 \mathrm{~mm}$ (2.5") diameter by $3.3 \mathrm{~mm}\left(1 / 8^{\prime \prime}\right)$ thick flyer plate.

In this study spall strength was calculated using an acoustic approach to relate spall strength to the pullback velocity observed at the back face as shown below in Equation (4.2) [52]:

$$
\sigma_{\text {spall }}=\frac{1}{2} \rho_{0} C_{0} \Delta u_{f s} .
$$

where $\rho_{0}$ is density, $C_{0}$ is bulk sound speed, and $\Delta u_{f s}$ is the difference between the peak and pullback free surface velocity of the sample. 


\subsubsection{Sound Speed Measurements}

Sound speed of the polyurethane composite samples was determined directly using a pulse echo transducer (Olympus Technologies 45 MG thickness gauge, M202-RM linear transducer, V157-RM shear transducer), shown in Figure 4.5. Pulse echo transducers use time of flight of an acoustic wave through a sample of known thickness to determine the wave speed. They are widely used by the shock physics researchers to determine bulk sound speed $[5,44,58,67]$. Using longitudinal and shear waves the respective longitudinal and shear sound speeds of the medium can be found [67]. From these wave speeds, the bulk sound speed of a material is found as $[44,58]$ :

$$
C_{0}=\sqrt{C_{\text {long }}^{2}-\frac{4}{3} C_{\text {shear }}^{2}}
$$

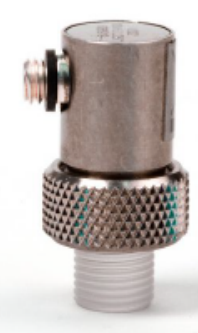

(a)

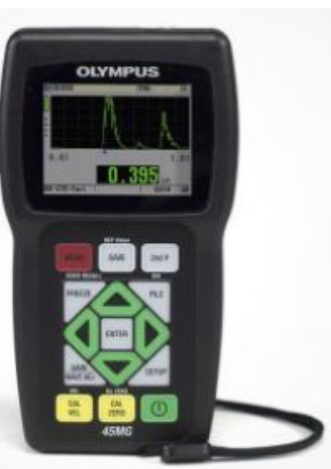

(b)

Figure 4.5: (a) Olympus M202-RM linear transducer and (b) Olympus 45 MG thickness gauge [68] 


\subsubsection{Geometry of the Sabot and Flyer Plate}

For all impact experiments, planarity (tilt) between the projectile and target faces is a priority to ensure the accuracy of the experimental results. As such, all mechanical components were designed with planarity in mind. Shown in Figure 4.6 are the projectiles used in these experiments consist of two parts, a flyer plate, and sabot. The flyer plate serves as an impactor against the target, while the sabot is a plastic projectile used to carry the flyer plate.

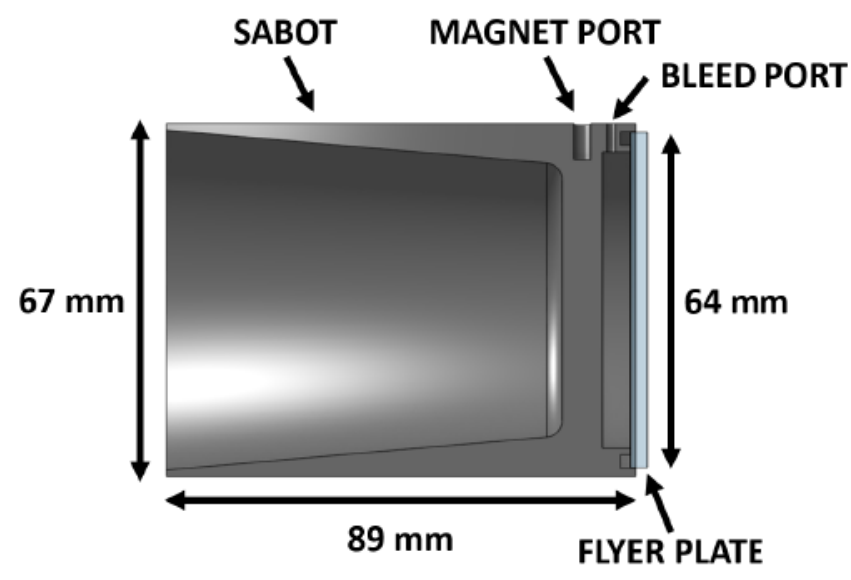

Figure 4.6: Labelled schematic of the sabot and flyer plate assembly

In the case of the sabot, planarity concerns meant analysing the effect of sabot length on yaw at the moment of impact. The trade-off being a longer projectile reduces possible yaw, yet is slower due to increased weight, and more costly to manufacture. Considering these influences, a function relating the projectile length to yaw was derived (Equation 4.4) and plotted in Figure 4.7 below to determine the optimal projectile length.

$$
L=\frac{D}{\sin (\theta)}-\tan ^{-1}(\theta) d-\tan (\theta) d+\frac{Z}{\cos (\theta)}
$$

where $\theta$ is tilt, $L$ is the length of the projectile, $D$ is diameter of the barrel, $d$ is projectile 


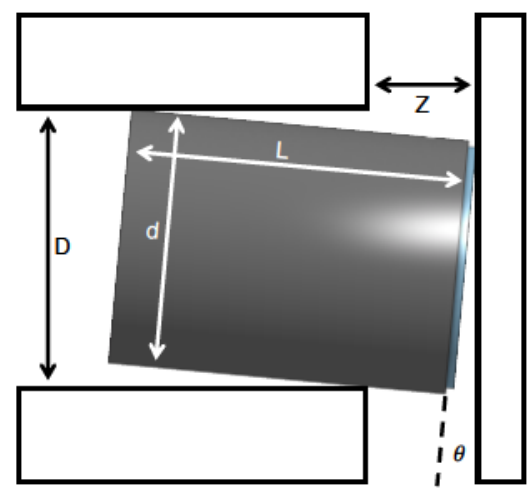

(a)

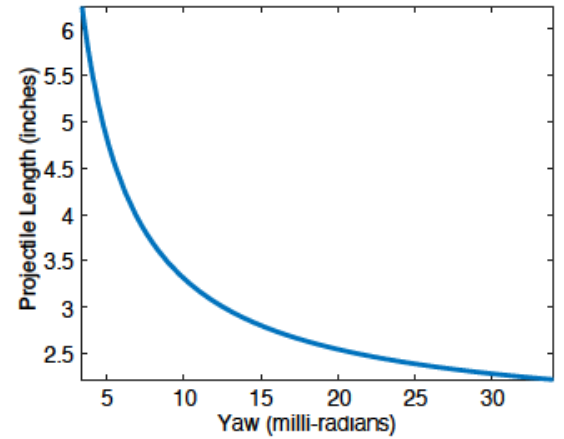

(b)

Figure 4.7: (a) Schematic and (b) plot for quantifying yaw of the projectile, as a function of projectile length

diameter, and $Z$ is the distance between the muzzle end and the impact surface. A projectile length of 3.5" was thus selected to yield the minimum yaw before the rate of return began diminishing. Equation 4.4 was determined using trigonometric relationships to derive $D$ and $Z$. $y$ denotes the length of the sabot remaining inside the barrel of the gas gun, measured parallel to $L$ from the muzzle. The equations are rearranged for $y$, then equated, yielding Equation 4.4 when simplified.

$$
\begin{aligned}
D & =\sin (\theta) y+\cos (\theta) d \\
Z & =\sin (\theta) d+\cos (\theta)(L-y)
\end{aligned}
$$

In addition to the effect of sabot length on tilt, the influence of sabot diameter on tilt can be seen. Sabot diameter was determined experimentally from a qualitative perspective. The barrel of the gas gun was measured to have a $67 \mathrm{~mm}$ (2.645") internal diameter. A oversized nylon plug with a length of approximately $60 \mathrm{~mm}$ long was manufactured for this purpose. The plug diameter was machined down incrementally until the plug could be smoothly driven down the entire length of the barrel, culminating in a final sabot diameter of $67 \mathrm{~mm}(2.635 ")$. 
Considering flyer plate geometry it can be seen that plate diameter is limited by the barrel diameter; a $64 \mathrm{~mm}(2.5 ")$ plate was used as it was the largest the $67 \mathrm{~mm}(2.635 ")$ diameter sabot could reasonably support. As discussed previously, the meeting of rarefaction fans within the target is a requirement for spall. From a simple 1D model of a symmetrical acrylic-acrylic impact backed with neat PU, the use of a $3.3 \mathrm{~mm}(1 / 8 ")$ thick acrylic flyer plate predicts a spall plane near the middle of the target for a range of impact velocities. This was validated experimentally, with a recovered neat PU target showing a well-defined spall plane, as seen in Figure 4.8.

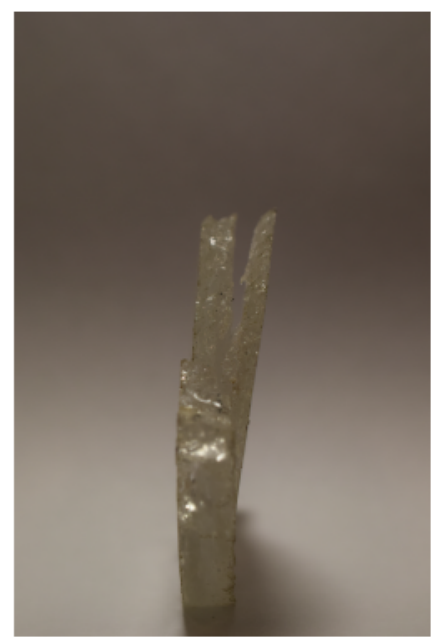

Figure 4.8: Observation of a clear spall plane in a recovered polyurethane sample

Considering flyer plate material, three options presented themselves as suitable candidates: acrylic, aluminum, and ceramic. Of some concern in this study was the possibility of elastic precursors resulting from an elastic-plastic, two-wave regime. Elastic precursors would generate more complex loading scenarios in the samples, making correlation of back face velocimetry to spall strength more difficult [19]. This issue can be addressed by inducing a one-wave regime in the experiment; plastic or elastic.

Ceramic flyers were considered for their high Hugoniot Elastic Limit (elastic loading), but were rejected due to their exorbitant cost (50-60\$ per plate). Inexpensive, acrylic plates 
were readily prepared from commercially available plate stock (Mcmaster Carr) through laser cutting performed by the School of Industrial Design at Carleton University. This combined with their low Hugoniot Elastic Limit, encouraging a wholly plastic regime, made them an ideal impactor material candidate. Laser cut, aluminum plates were also used as impactors to vary loading conditions, without changing striking velocity. Due to their higher elastic limit, the aforementioned elastic precursors were a concern, but were not observed in the PU experiments presented in this study.

\subsubsection{Target Assembly}

Alongside the sabot and flyer plate, design of the target assembly was performed with planarity in mind, ensuring all mating surfaces were flat and planar. Implications of target assembly, target mounting, instrumenting the target, and maximizing re-usability were considered. For reference, an image of the target assembly, mounted upon the muzzle end of the gas gun can be seen in Figure 4.9. Furthermore, Figures 4.10 and 4.11 present schematics of the respective PU and PDMS target assemblies.

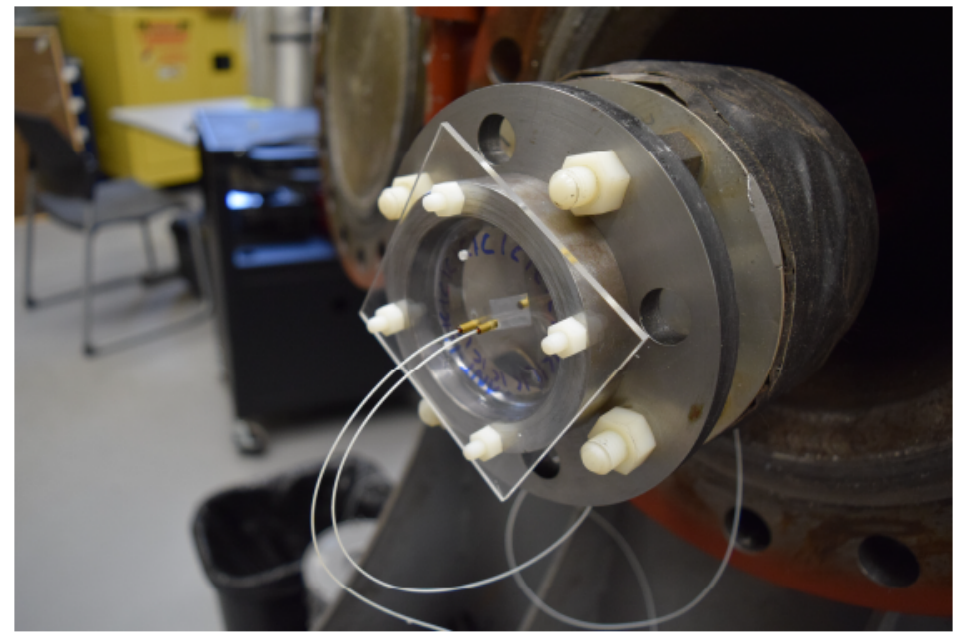

Figure 4.9: Target assembly mounted to the muzzle of the barrel and instrumented with two PDV probes 
Prior to any assembly, a piece of aluminized Mylar film was affixed to the centre of the sample backface using cyanoacrylate. Care was taken to ensure the aluminized surface was smooth and lay squarely beneath both PDV probe locations. Assembly of the target was accomplished by epoxying the integrated striker plate/sample unit into a machined side wall and mounting flange. The assembly was then bolted together using nylon machine screws and nuts. Lastly, PDV probes were mounted into the target assembly via an acrylic plate affixed to the aft end of the sidewall.

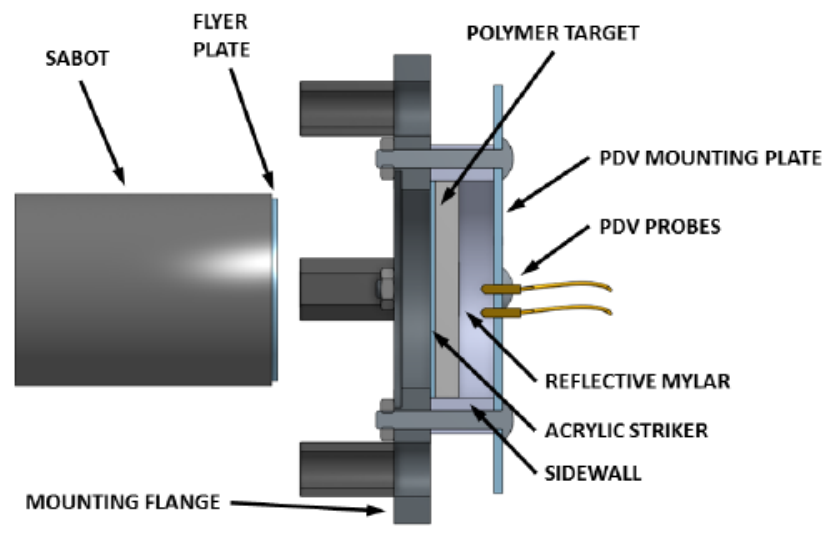

(a)

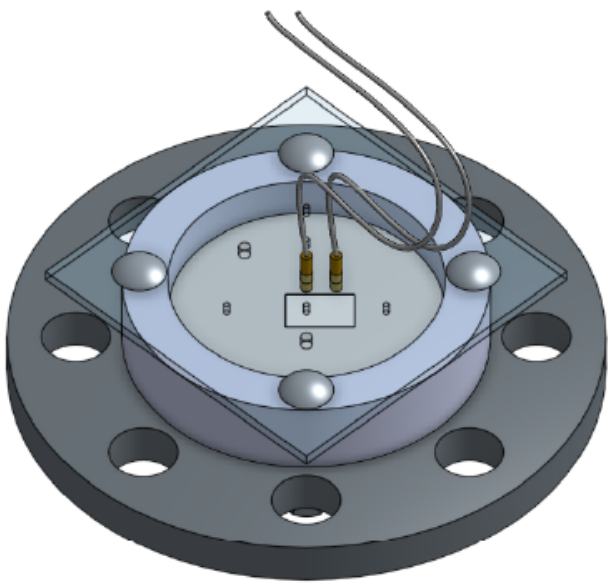

(b)

Figure 4.10: Schematic representations of the experimental apparatus used for the polyurethane based samples; (a) labelled side view and (b) isometric view

An assembled target is then mounted to the SSLGG using 1/2"-13 nylon threaded rod and nuts, with precision washers and threaded standoffs facilitating planar mounting. As a final check, digital calipers measured offset from the muzzle while tightening the nuts to maximize planarity. Nylon mounting hardware was deliberately used as sacrificial, low cost components; the nylon hardware fails first, reducing the risk of plastic deformation in the machined steel components, which are costly to replace. 


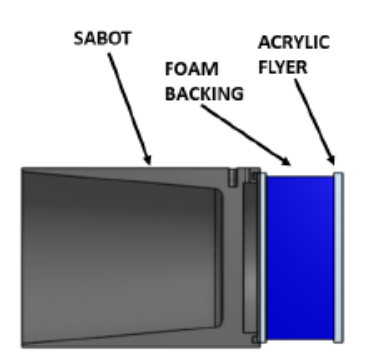

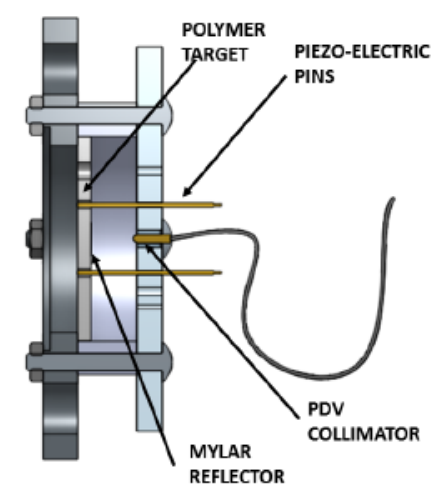

(a)

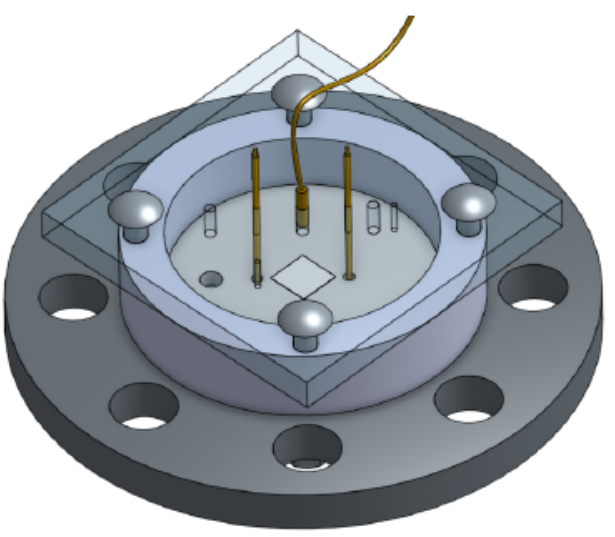

(b)

Figure 4.11: Schematic representations of the experimental apparatus used for the PDMS based samples; (a) labelled side view and (b) isometric view

\subsection{Experimental Setup of the PDMS Study}

The PDMS samples were subjected to a thin flyer plate impact to induce a dynamic tensile loading condition at its midplane. The flyer plate was launched using a $67 \mathrm{~mm}$ internal diameter smooth-bore single-stage gas gun. Samples were cast to a thickness of approximately $5 \mathrm{~mm}$ and adhered to a $0.25 \mathrm{~mm}(0.010 ")$ thick Mylar film with cyanoacrylate. The Mylar film on the front face of the target was used to address concerns of the flexible PDMS samples bowing under their own weight. Schematics of the target assembly and sabot assembly are shown in Figure 4.11.

Targets were instrumented with piezo-electric pins (shock pins) and a two-channel photonic Doppler velocimeter (PDV) [66]. Like the polyurethane experiments described before, an aluminized Mylar foil adhered to the back face of the sample with cyanoacrylate served as a reflector for a PDV laser collimator, allowing for observation of back face velocity. Two PDV probes were used in each experiment for redundancy.

Two time-of-arrival shock pins (model CA-1135 from Dynasen Inc.) were mounted at a radius of $12.5 \mathrm{~mm}(1 / 2 ")$ from the target center, flush with the front face of the sample 
to provide an average time of initial impact, as well as one dimension of tilt between the sample and the flyer plate. Shock pins are piezoelectric devices that generate a voltage when loaded via an external force. In the case of plate impact testing this load comes from the shock wave generated by the experiment. As the shock wave arrives at the shock pins and loads the pin a corresponding signal is generated in the pin. Planarity of the impact can be determined using Equation 4.5 shown below:

$$
\theta=\sin ^{-1}\left(\frac{V_{\text {projectile }} * \Delta T}{D}\right)
$$

where $\theta$ is the angle of tilt in a given direction, $\Delta T$ is the difference between time of arrival of the two pins, and D is the lateral distance between the pair of shock pins. Figure 4.12 depicts the shock pin voltage traces over time as observed by an oscilloscope during an experiment. The sharp rise in each trace around $1.5 \mu$ s denotes the time of impact for the respective pin.

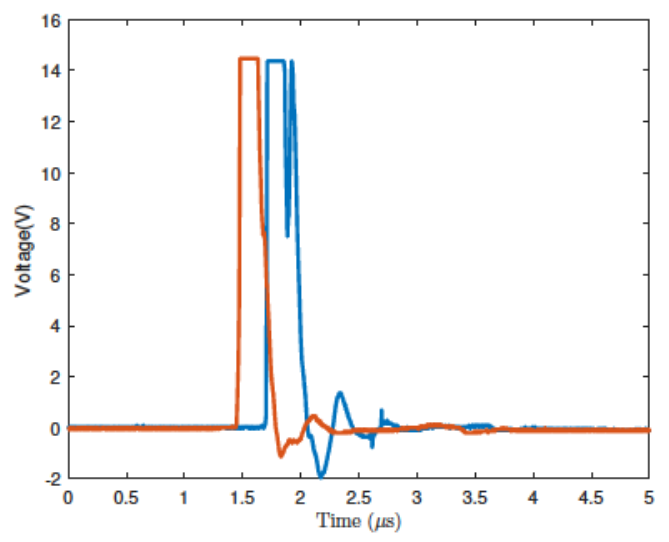

Figure 4.12: Shock Pin trace of a PDMS spall experiment corresponding to a tilt of 2.8 milli-radians

Shock pins were removed from later experiments (spall experiments in PU) due to cost, and the fact tilt was found to be negligible; typically 30 mill-radians $\left(\approx 1.7^{\circ}\right)$ or less.

The same $89 \mathrm{~mm}$ (3.5") sabot described earlier was used to carry to the flyer plates used 
in this study. Samples were impacted with a $64 \mathrm{~mm}(2.5 ")$ diameter by $3.3 \mathrm{~mm}(1 / 8 ")$ thick acrylic plates. These flyer plates were backed by $25.4 \mathrm{~mm}$ (1") thick diameter matched open-cell polyurethane foam backing (Mcmaster Carr). The foam backing was used to mitigate concerns regarding bowing of the acrylic plate, from inertial forces during acceleration prior to impact. Foam was selected as the backing material due to its low impedance relative to the acrylic, behaving as a free surface when the initial shockwave reaches the foam-acrylic interface. After the PDMS study, it was found in subsequent experiments that the $3.3 \mathrm{~mm}(1 / 8 ")$ acrylic plates without the foam, generated high quality data, suggesting the flyer plates did not bend in a manner sufficient to cause issues with spall.

\subsection{Test Matrix}

Having introduced both the polyurethane and PDMS studies, each with their own varying parameters, it is worth discussing the design envelopes of both studies. As can be seen in Table 4.2, the PDMS study used the spherical filler, with two silane treatments, in order to broaden the spectrum of adhesion investigated. With a total of three sample types, it can be seen that this study focused on the effects of interface adhesion.

\begin{tabular}{|l|c|}
\hline Silane Treatment & Spherical Filler \\
\hline Increased Adhesion & $\mathrm{X}$ \\
Untreated & $\mathrm{X}$ \\
Impeded Adhesion & $\mathrm{X}$ \\
\hline
\end{tabular}

Table 4.2: Test matrix of the PDMS study

Table 4.3 shows the test matrix for the polyurethane. Here, the polyurethane study, included the neat polyurethane in the study, eliminated the silane used to improve adhesion, and integrated the pristine, irregular, alumina filler into the test matrix. Compared to the 
PDMS, the focus of this study was broader, integrating sample types to investigate the effects of polymer reinforcement, particle morphology effects, and surface adhesion effects.

\begin{tabular}{|l|c|c|c|}
\hline Silane Treatment & Neat & Spherical Filler & Irregular Filler \\
\hline Increased Adhesion & & & \\
Untreated & $\mathrm{X}$ & $\mathrm{X}$ & $\mathrm{X}$ \\
Impeded Adhesion & $\mathrm{X}$ & $\mathrm{X}$ & \\
\hline
\end{tabular}

Table 4.3: Test matrix of the polyurethane study

\subsection{Instrumentation and Data Processing}

The Section will introduce the instrumentation used throughout this study and provide an introduction to methods used to obtain results from each of the instruments.

\subsubsection{Annular Coil Gauges}

Of significant interest to this study is the muzzle velocity of the flyer plate prior to impact. Two annular coil gauges were used to measure muzzle velocity. The predecessor to the Carleton gas gun at Mcgill University uses transverse coil gauges [31,69], but the decision to use annular coil gauges at Carleton came from difficulties in generating a signal during bench top testing. The annular design readily generated a much more powerful signal.

A coil gauge operates on Faraday's Law, the principle that a voltage will be generated in a conductor as a result of a changing magnetic field [70]. In this case the changing magnetic field is from a magnet mounted on the fast moving sabot, and the conductor is a stationary coil of magnet wire. As the magnet bearing projectile approaches the coils, a voltage is induced in the coils from the moving magnetic field. The voltage continues to 
increase with the approaching magnet, reaching a peak when the magnet crosses the coil centre-line. As the magnet passes the coil centre-line, the direction of the magnetic field experienced by the coil inverts due to the geometry of the magnetic field, sharply inverting the voltage in the coil. This inversion signals the instant the magnet is present at the coil centre-line. Using a pair of coil gauges at a known offset $(60.33 \mathrm{~mm} \pm 2.38 \mathrm{~mm})$ the muzzle velocity is determined using:

$$
V=D / T
$$

where $D$ is the distance between the centres of the coil gauges and $T$ is the time between signal cross-over of the two coil gauges. Two concerns regarding the coil gauge system were accuracy and terminal acceleration of the sabot between the coil gauges and impact face of the target assembly. These concerns were addressed through an experiment where the sabot was monitored concurrently with the coil gauge system and a laser velocimetry system. As seen in Figure 4.13, the shot yielded a velocity of $259 \mathrm{~m} / \mathrm{s}$ and $263 \mathrm{~m} / \mathrm{s}$ for the coil gauges and laser system respectively. Furthermore, observation of the trace for the 200 $\mu$ s (travel distance of approximately $50 \mathrm{~mm}$ ) after passing the second coil gauge shows a consistent velocity for the sabot, validating the accuracy of the coil gauges.

\subsubsection{Photonic Doppler Velocimetry}

As a data acquisition tool, photonic Doppler velocimetry (PDV) observes the velocity of a reflective surface of interest using an infrared laser and the concept of Doppler shifting [71]. The ability to monitor surface velocity proves useful in spall experiments, as the free surface velocity response correlates to directly spall strength. Taking advantage of this relationship, PDV is used to determine spall strength. In essence, a laser of 


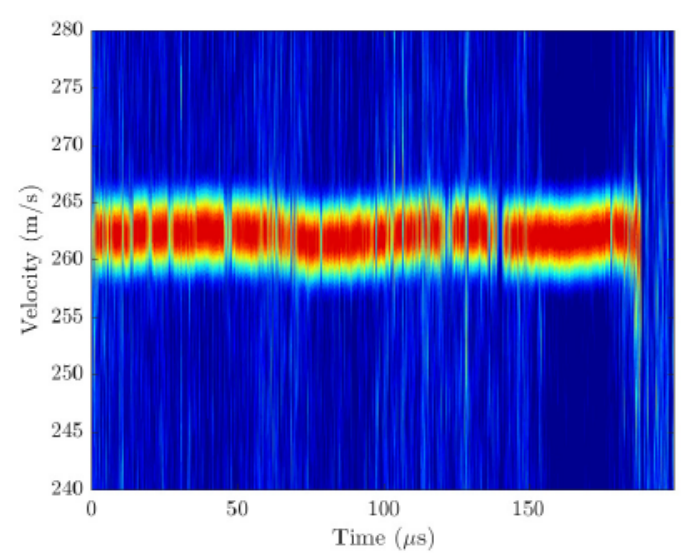

(a)

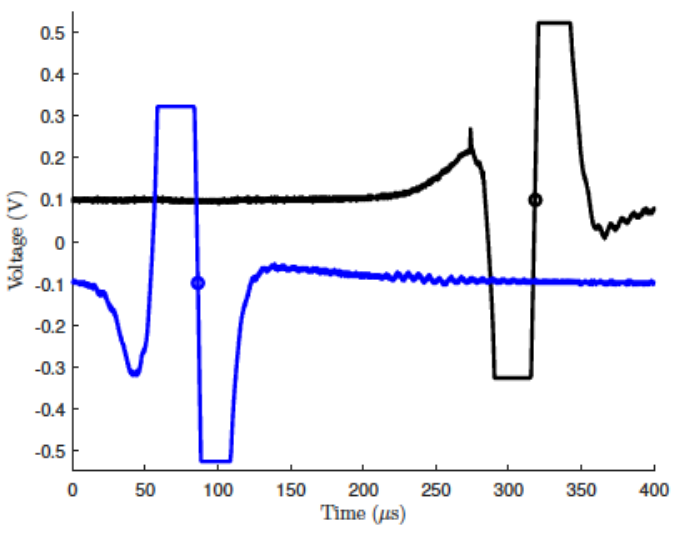

(b)

Figure 4.13: (a) Laser velocimetry and (b) coil gauge instrumentation observing the same projectile, recording a velocity of $263 \mathrm{~m} / \mathrm{s}$ and $259 \mathrm{~m} / \mathrm{s}$ respectively.

known frequency is split, with one half retained and the other emitted at a moving surface of interest. The retained beam is combined with the reflected beam whose frequency has been Doppler shifted from reflecting off a moving surface [71]. The process of splitting, emitting, collecting, and combining the beam is shown in Figure 4.14.

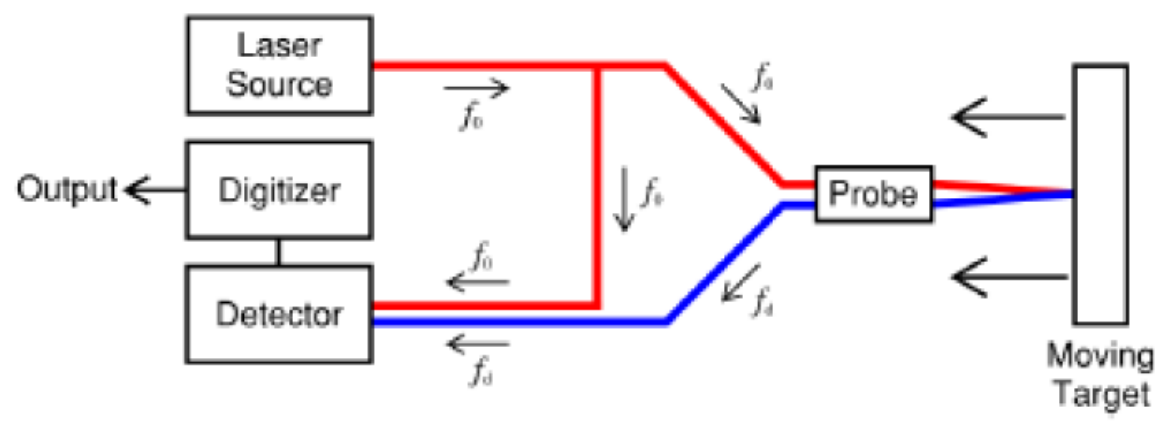

Figure 4.14: Schematic of PDV setup [66]

The difference in the emitted and retained laser frequency is referred to as the beat frequency and is directly related to the velocity of the moving object. Next, a sliding window Fast Fourier Transform (FFT) to converts the raw data to a usable velocity, profile allowing for effective analysis of the velocity [71]. Examples of pre- and post-processing 
traces are shown in Figure 4.15. The drastic increase in signal frequency, shortly after $5 \mu \mathrm{s}$ in Figure 4.15 (a) can be seen to coincide with the arrival of the shockwave at the same time in Figure 4.15 (b).

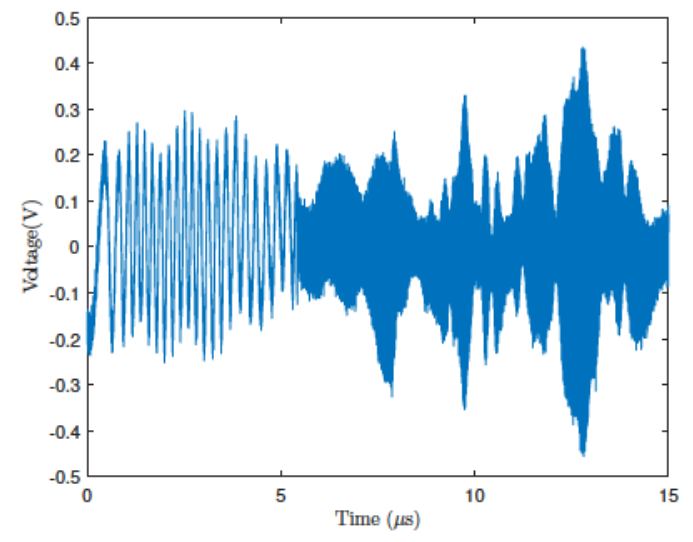

(a)

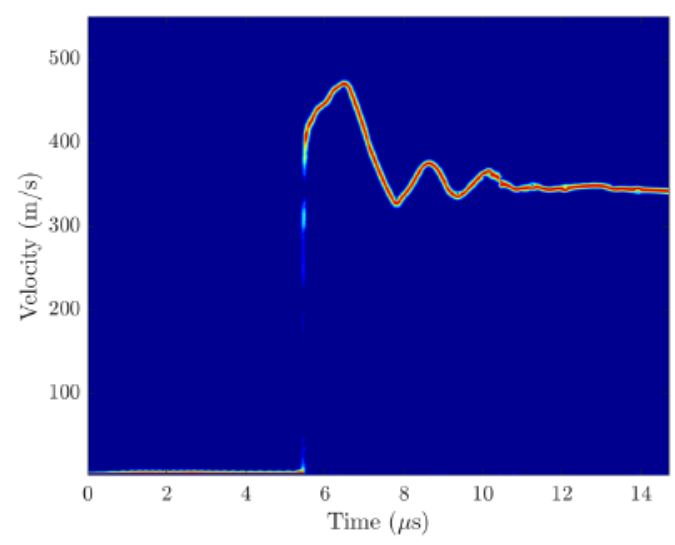

(b)

Figure 4.15: (a) Raw PDV data as displayed on our oscilloscope compared and its (b) free surface velocity spectrogram after FFT processing

The PDV system at Carleton uses a $1550 \mathrm{~nm}$ laser, manipulated with an optical board used to mix and control the path of the laser. The combined optical signal is converted to an electric signal with a photo-detector, then digitized by oscilloscope. More specifically, the PDV system records the signal with a $12.5 \mathrm{GHz}$ photo-detector, digitized by oscilloscope at a sample rate of $40 \times 10^{9}$ samples per second. The raw data is processed using a sliding window Fast Fourier Transform (FFT) with a window size of 10,000 data points and a time resolution of $50 \mathrm{~ns}$. Window size can be increased to give a finer velocity resolution at a cost of reduced time resolution.

Experiments are monitored by PDV collimators, which consist of a length of fibre optic cable fused to a probe head. Within the probe head is a lens used to ensure the light remains aligned in a tight beam (collimated). To provide redundancy, two PDV collimators are used in each experiment; one monitoring the centre of the sample back face, and one offset from 
the center by a radius of $9.5 \mathrm{~mm}\left(3 / 8^{\prime \prime}\right)$. The collimators possess a focal length of $1 \mathrm{~cm}$, encouraging a smaller offset between the head of the collimator and the Mylar surface. However, a sufficient offset is required so that the collimator head is not destroyed by the fast moving free surface, prior to the completion of the experiment.

Mounting of the probes is a delicate process. Prior to use, the FC/APC connector of each probe is cleaned with a fibre cleaning tool, then inspected for damage or dirt using a fibre microscope. Damaged probes are discarded and replaced. Using lint free swabs and alcohol, the tip of each collimator is gently cleaned to remove any debris that could interfere with the beam of the laser. Placing the probe in its mounting hole, a laser leak detector is used to generate a visible beam, in turn used for aligning the probe. A probe is considered well aligned if no scattered light is observed, and a singular red point is seen on the aluminized Mylar. In some cases, the portion of fibre fused to the collimator head would glow red from the reflected light, which is taken to suggest good alignment. A small drop of cyanoacrylate is then used to adhere the collimator in place, taking care to avoid having any adhesive touch the collimator lens. After connecting the probe to the rest of the PDV system and upon powering up the laser, power monitors observing the power intensity of the signal returned from the collimators are inspected. A power return greater than $-30 \mathrm{dBm}$ has been found to yield good results, with higher intensities typically yielding better spectrograms. Abnormally high power returns $(>-10 \mathrm{dBm})$ are typically a consequence of a bad probe, which is replaced prior to performing an experiment. 


\section{Chapter 5}

\section{Results and Discussion}

The purpose of this Chapter is to discuss the findings of the PDMS and PU studies. Specifically, this chapter will present the data collected from these experiments, then discuss the significance of those results. Literature discussed previously will be used to relate the results presented here to existing bodies of work, as well as to support the conclusions drawn from these studies.

\subsection{Spall in PDMS Systems}

This Section presents and discusses the results of spall experiments performed on the suite of PDMS samples. The bulk of this section has been peer reviewed, as part of an accepted conference proceedings manuscript, submitted to the 2019 Topical Group on Shock Compression of Condensed Matter, within the American Physical Society. A selection of spectrograms from this study are shown for each sample type in Figures 5.1-5.3.

A summary of the spall strengths measured for the samples, each tested in duplicate, can be found in Table 5.1. These spall strengths were determined from the backface velocity history of the samples during plate impact testing, based on Equation 4.2. The results showed an interesting trend with respect to the surface treatments applied to the particles. The baseline untreated samples had spall strengths of 56.4 and $51.3 \mathrm{MPa}$, the positivelytreated samples had spall strengths of 48.7 and $43.4 \mathrm{MPa}$, and the negatively-treated samples had spall strengths of 21.4 MPa and 16.4 MPa. 


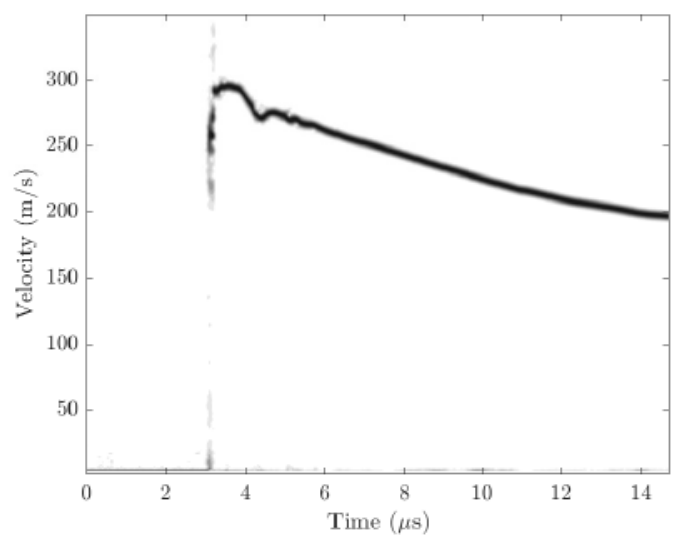

(a)

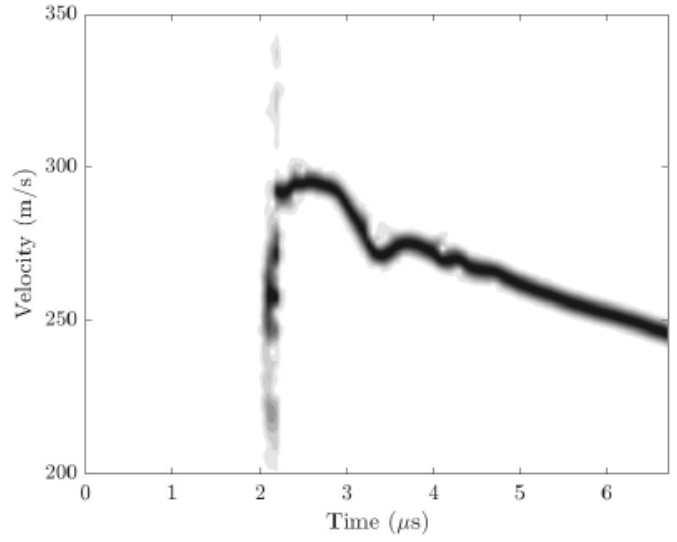

(b)

Figure 5.1: (a) PDMS-Alumina target struck by an acrylic flyer at $282 \mathrm{~m} / \mathrm{s}$ (b) same impact as shown in (a) cropped to focus on peak and pullback velocities

\begin{tabular}{|c|c|c|c|}
\hline Sample & Impact Velocity $(\mathrm{m} / \mathrm{s})$ & $\Delta u_{f s}(\mathrm{~m} / \mathrm{s})$ & Spall Strength $(\mathrm{MPa})$ \\
\hline PDMS+AL \#1 & 281.9 & 23.3 & 56.4 \\
PDMS+AL \#2 & 284.1 & 21.2 & 51.3 \\
\hline PDMS+AL POS \#1 & 311.6 & 20.1 & 48.7 \\
PDMS+AL POS \#2 & 285.1 & 17.9 & 43.4 \\
\hline PDMS+AL NEG \#1 & 282.5 & 8.9 & 21.4 \\
PDMS+AL NEG \#2 & 292.0 & 6.7 & 16.2 \\
\hline
\end{tabular}

Table 5.1: Results of plate impact testing of PDMS composites

Surprisingly, the results show that there is a slight decrease in the spall strength of the composite when comparing the untreated samples with the samples treated for improved adhesion. While the untreated samples with a strength of 56.4 and $51.3 \mathrm{MPa}$ appear to be stronger than the positively-treated samples at 48.7 and $43.4 \mathrm{MPa}$, the differences are considered to be small enough to be indicative of equivalent strength materials. Differences in the spall strengths between these two sample sets were taken to be a result of inherent spread between experimental trials.

Alternatively, this may be an indication that the active surface chemistry of the alumina 


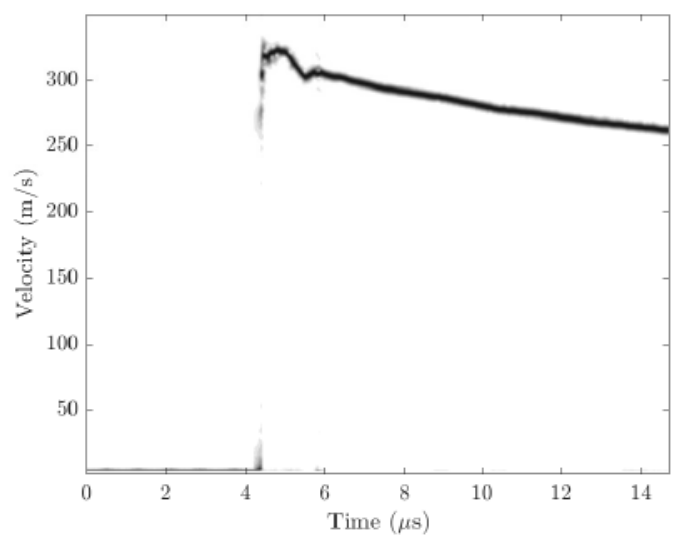

(a)

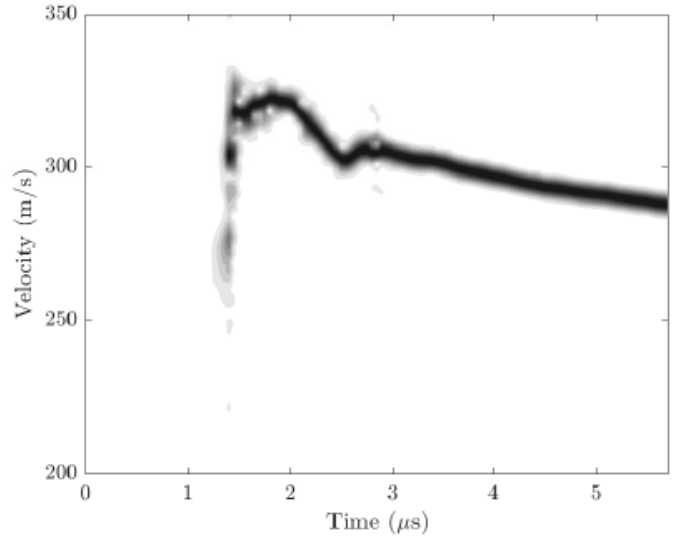

(b)

Figure 5.2: (a) Positively-treated PDMS-Alumina target struck by an acrylic flyer at $312 \mathrm{~m} / \mathrm{s}$ (b) same impact as shown in (a) cropped to focus on peak and pullback velocities

particles results in sufficiently strong adhesion to the PDMS, such that the silane treatment did not specifically improve the adhesion seen and may have even resulted in a slight degradation.

Comparison of the measured spall strengths of positively- and negatively-treated samples can be seen in Table 5.1, showcasing the apparent influence of interface adhesion on spall strength. In this case, negative treatment produced a spall strength that was consistently and notably reduced in comparison to the other sample types. It should be noted that the two sample types are identical except for the surface treatments applied to the alumina filler. As such the difference in spall strength can be attributed directly to the influence of the silane treatments. As discussed by Antoun et al [11], a reduced magnitude of the pullback pulse correlates to a lower stress required to nucleate voids. This was the case for the negatively treated samples presented here. The higher spall strength of the other samples suggests that the presence of interface adhesion between the two phases was responsible for impeding the void initiation process in the material. 


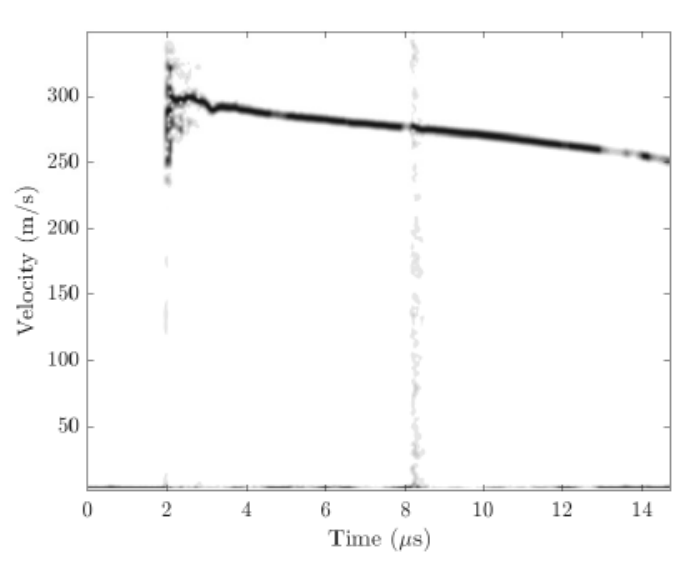

(a)

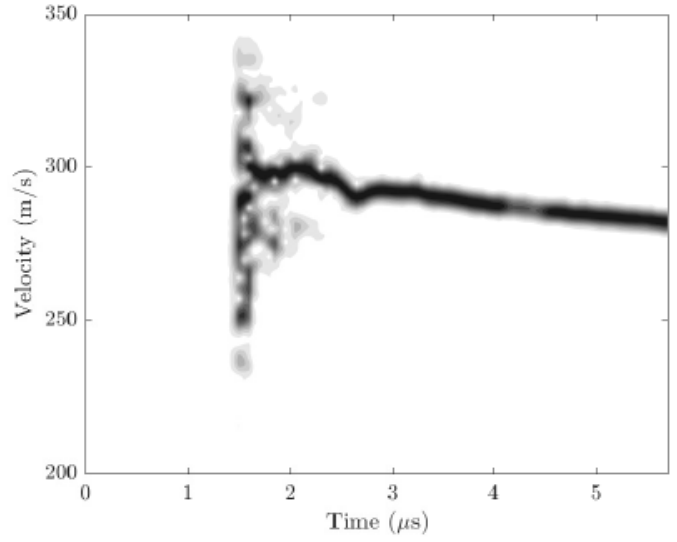

(b)

Figure 5.3: (a) Negatively-treated PDMS-Alumina target struck by an acrylic flyer at $283 \mathrm{~m} / \mathrm{s}$ (b) same impact as shown in (a) cropped to focus on peak and pullback velocities

In presenting the spectrograms of PDMS samples undergoing spall experiments, some concern arises from the apparent noise that can be seen in Figure 5.3 (b) between $\sim 1.5$ and $2.5 \mu \mathrm{s}$. This "noise" around the arrival of the shock wave suggests that the sample may be prematurely fracturing, or emitting ejecta. Upon investigation, these concerns were put to rest, with the "noise" attributed to harmonics in the FFT spectrogram. Due to the nature of analyzing the frequency based response of a sample by PDV, harmonics are a common occurrence in spectrograms.

Harmonics can be seen in the spectrograms of PDV data presented in other literature $[72,73]$. As such, harmonics are thought to be the source of vertical noise seen at the arrival time of the shockwave. Observing the spectrogram to a peak velocity of $700 \mathrm{~m} / \mathrm{s}$ this harmonic can be seen in Figure 5.4 (a). The presence of this second harmonic and its rise overlap with the spectrogram presented. Furthermore, past experiments where the back face of the sample generated ejecta (break away of a sputter coated reflector on the free surface) showed a much more intense and longer enduring cloud of noise in the spectrogram [32], as shown in Figure 5.4 (b). The noise seen around the time of breakout in 
Figure 5.3 is localized with a very short duration.

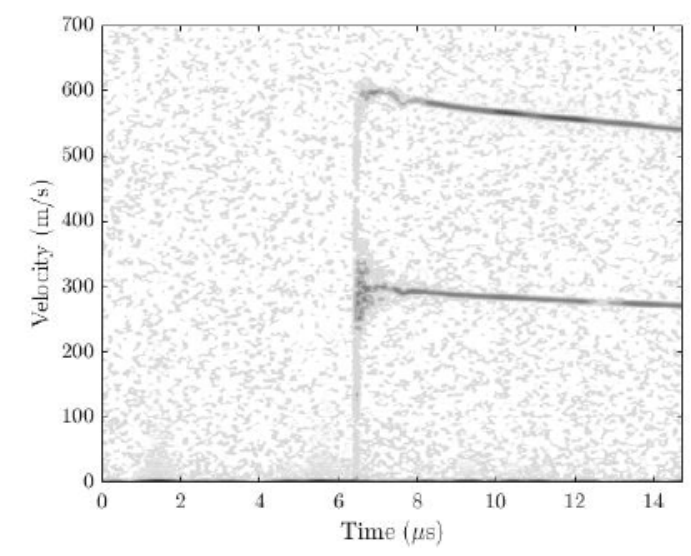

(a)

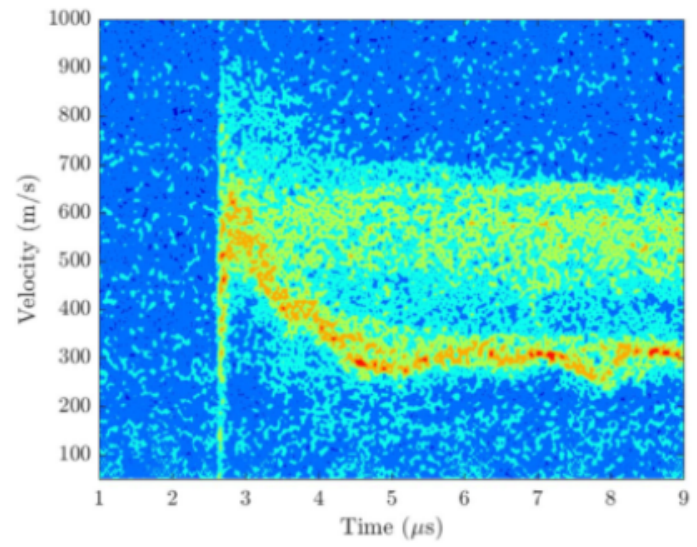

(b)

Figure 5.4: Spectrograms of spall experiments showing: (a) resonance (b) the noise generate by ejecta generated at the back face [32]

One further concern was the possible separation of the reflector from the target. As discussed previously, these experiments used commercial aluminized Mylar film adhered to the back face of the sample as a reflector for the incident beam of the PDV system. If that were to have separated from the sample it is expected the Mylar would have an independent motion with minor reverberations in the free surface velocity due to waves travelling in the thin film. Shown in Figure 5.5 is the PDV trace of an epoxy sample under plate impact conditions that likely ejected the reflective Mylar. Evidence supporting this is the high frequency reverberations starting around $2.5 \mu$ s, which is likely a consequence of waves rapidly traversing the extremely thin Mylar film.

In this Section the effect of altering the adhesion between the phases of a composite on the spall strength of the bulk composite was experimentally investigated. Silane treatments were applied with the intent of increasing or impeding adhesion between particle phase and the PDMS matrix. Compared to the untreated samples, a slight reduction in the spall 


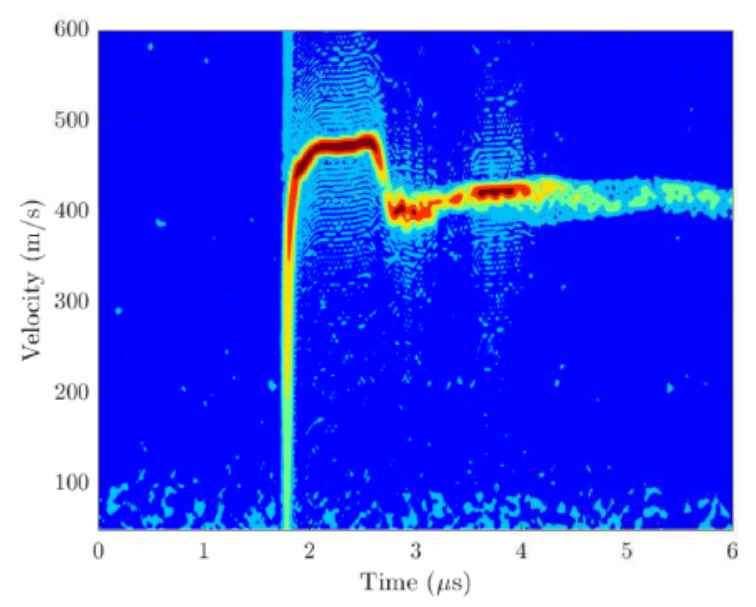

Figure 5.5: PDV trace suggesting the ejection of the aluminized Mylar from the back face of an epoxy sample

strength was observed in the samples treated for improved adhesion. As discussed, this minor change was considered within experimental variation, but it may be evidence of an inherently active surface chemistry of the pristine alumina. A comparison of the composites with improved and impeded adhesion showed a drastic reduction in strength associated with a loss of adhesion at the particle surface.

\subsection{Polyurethane Spall Testing}

Having presented the findings of the PDMS spall study, the results of the PU spall study will now be addressed. The discussion will begin with density and sound speed measurements of each sample type. Results of the spall experiments will then be presented, beginning with the neat PU, followed by an assessment of morphology effects, and culminating with the influence of matrix-filler adhesion on spall strength. For reference purposes, Appendix A contains the compiled and tabulated results of the PU spall experiments. 


\subsubsection{Sound Speed and Density Measurements}

To accurately apply the spall strength equation, the density and sound speed of each sample type was determined experimentally, and compared to the theoretically estimated values. As can be seen in Table 5.2, density was found for each sample type, with at least four samples of each type measured. The theoretical density of the composites was determined as the sum of the products of the density (measured density of the neat polyurethane was used) and volume fraction of each phase. Density of the samples was measured for two reasons: to provide an accurate density for use in spall strength calculations, and as a way to check for the presence of entrained air that could compromise the performance of the samples. Entrained air may behave as a pre-existing flaw that could artificially reduce spall strength $[15,22,31]$.

\begin{tabular}{|c|c|c|}
\hline Sample & Measured Density $\mathrm{g} / \mathrm{cm}^{3}$ & Theoretical Density $\mathrm{g} / \mathrm{cm}^{3}$ \\
\hline Neat Polyurethane & $1.09 \pm 0.03$ & 1.04 \\
\hline Spherical $\mathrm{Al}_{2} \mathrm{O}_{3}$ & $1.66 \pm 0.04$ & 1.66 \\
\hline Irregular $\mathrm{Al}_{2} \mathrm{O}_{3}$ & $1.69 \pm 0.04$ & 1.66 \\
\hline Silane Treated & $1.60 \pm 0.04$ & 1.66 \\
\hline
\end{tabular}

Table 5.2: Average measured and theoretical density of each sample type

The theoretical density of $1.04 \mathrm{~g} / \mathrm{cm}^{3}$ listed for the Neat Polyurethane is the density provided by the manufacturer (Smooth On) of Clearflex 95. With a measured density slightly higher than the published density, it can be said with reasonable confidence that the neat polyurethane is devoid of bubbles. The higher density is of little concern and lies within the bounds of uncertainty. Shrinkage observed in the samples after post-curing at an elevated temperature (optional step in preparation instructions provided by manufacturer) may be to blame for the slightly higher than expected density.

The spherical alumina reinforced PU possesses a density equal to its theoretical density. 
As discussed before, this is considered to be a positive finding as it suggests the samples are devoid of pores/voids that could weaken the overall composite. Thirdly, the irregular alumina reinforced PU possesses a density slightly above its theoretical density, which is considered to be of little concern as it lies within the bounds of uncertainty, and also suggests the absence of pores/voids.

The density of $1.60 \mathrm{~g} / \mathrm{cm}^{3}$ listed for the silane-treated samples refers to the density of two batches made separately for further spall testing. Both batches fell within the listed bounds of uncertainty. The minor differences between the measured and theoretical densities for these silane-treated samples fall slightly outside the bounds of measurement uncertainty. However, the lower measured density in the silane-treated samples can be attributed to the 5\% mass fraction of silane (relative to the alumina) present in the treated powder. The lower overall density of the treated alumina would propagate through to the final sample density as the samples were prepared in batches with each ingredient added by mass.

In considering density, it is also worth addressing the possibility of settling of the dense filler in the samples during manufacture. With an elapsed manufacturing time of about 24 hours there is ample time for the dense alumina particles to sink into the still liquid polyurethane matrix. The absence of settling was confirmed through X-ray imaging and cross-sectional analysis of the samples after manufacturing. X-ray imaging of the sample in Figure 5.6 (a) shows a constant attenuation through the height of the sample suggesting that no settling had occurred. If settling was present, it is expected that the base of the samples would appear dark relative to the top, due to the increased attenuation of the dense alumina. Likewise, cross-sectional cutting in Figure 5.6 (b) observed a constant appearance and consistency through the samples, further ruling out settling.

Next to be discussed is speed of sound in these samples. As stated, the theoretical sound 


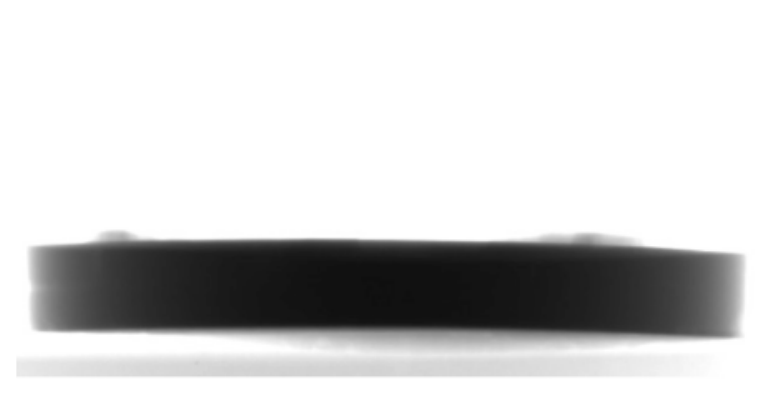

(a)

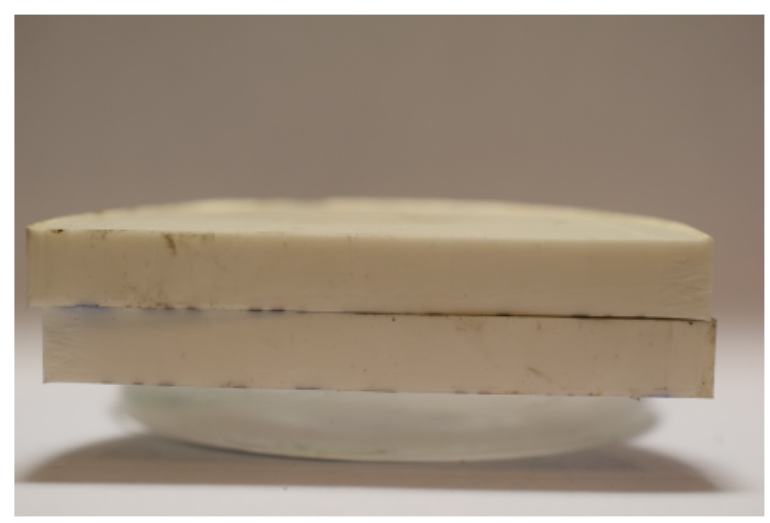

(b)

Figure 5.6: Cross-sectional views demonstrating a lack of settling in the alumina filler via $\mathrm{X}$-ray (a) and optical imaging of a section (b)

speed of a material can be defined as:

$$
C=\sqrt{\frac{E}{\rho}}
$$

where $E$ is Young's modulus and $\rho$ is density. In turn, the theoretical Young's modulus of the composite can be determined using the Reuss model for composites shown in Equation 3.11. Density was taken from the measured density of the samples shown above in Table 5.2. In using the Reuss model, density of the neat polyurethane was taken as the measured density of $1.09 \mathrm{~g} / \mathrm{cm}^{3}$ with Young's modulus determined using the published Young's modulus of ClearFlex 95 (Shore 95A Hardness). The properties of the alumina filler were taken to be: $215-413 \mathrm{GPa}$ for Young's modulus, and $3.95 \mathrm{~g} / \mathrm{cm}^{3}$ for density [74]. In using the Reuss Model (Equation 3.11) to predict density, it was observed that a Young's modulus of 215 or $413 \mathrm{GPa}$ for the alumina filler did not change the predicted sound speed of the composites.

From Table 5.3 a few interesting observations arise. The measured and theoretical sound speed of the neat polyurethane can be seen to be almost identical. Likewise the 


\begin{tabular}{|c|c|c|}
\hline Sample & Measured Sound Speed $\mathrm{km} / \mathrm{s}$ & Theoretical Sound Speed $\mathrm{km} / \mathrm{s}$ \\
\hline Neat Polyurethane & $2.17 \pm 0.01$ & 2.16 \\
\hline Spherical $\mathrm{Al}_{2} \mathrm{O}_{3}$ & $2.09 \pm 0.01$ & 1.92 \\
\hline Irregular $\mathrm{Al}_{2} \mathrm{O}_{3}$ & $2.06 \pm 0.01$ & 1.90 \\
\hline Silane Treated & $1.99 \pm 0.02$ & 1.94 \\
\hline
\end{tabular}

Table 5.3: Average measured and theoretical sound speeds of each sample type

measured and theoretical sound speed of the silane-treated sample can be seen to be very similar. Regarding shape, Table 5.3 shows that the measured sound speed of the spherical and irregular alumina reinforced samples are quite comparable. This suggests filler shape does not change the extent to which the filler influences the elastic wave response of the PU composite.

However, it is also interesting to observe that measured sound speeds for the spherical and irregular alumina reinforced samples are slightly higher than that of the negatively treated samples even though all three samples contain essentially the same volume fraction of alumina. Mixture models like the Reuss models (Equation 3.11) directly consider only Young's modulus and volume fraction of the relative phase to predict Young's modulus of the composite. This Young's modulus alongside mixture density are used to predict sound speed as per Equation 5.1. In this study, it is believed adhesion is responsible for the change in measured sound speed between the different alumina composites. As seen before, increased matrix-filler adhesion tends to increase Young's modulus of a composite [36,38]. An increased Young's modulus would increase the sound speed of the composite as per Equation 5.1. Taking this a step forward and converting the measured densities and sound speeds to a theoretical Young's modulus the following is observed in Table 5.4.

As can be seen the spherical and irregular samples show little variation in Young's modulus (due to the comparable sound speeds and densities) as opposed to the reduction 


\begin{tabular}{|c|c|}
\hline Sample & Estimated Young's Modulus (MPa) \\
\hline Spherical $\mathrm{Al}_{2} \mathrm{O}_{3}$ & $7.25 \pm 0.25$ \\
\hline Irregular $\mathrm{Al}_{2} \mathrm{O}_{3}$ & $7.17 \pm 0.27$ \\
\hline Silane Treated & $6.33 \pm 0.28$ \\
\hline
\end{tabular}

Table 5.4: Estimated Young's modulus of each alumina reinforced sample type as a function of measured density and sound speed

in Young's modulus seen in the negatively treated alumina. This shows in one way how interface adhesion may play a role in the stiffness of a composite [38]. Poor adhesion in the negatively treated sample may be to blame for a reduction in overall Young's modulus, by suppressing the extent to which the alumina filler is able to interact with the matrix and reinforce the overall composite even though filler geometry, size, shape, and effective volume fraction are kept consistent.

\subsubsection{Spall in Neat Polyurethane}

The discussion of spall strength will begin with the results of the neat PU. This Section will discuss the spall strength of the PU, relate it to other polymers presented in comparable literature, and discuss the behaviour of the PU under varying strain rates. Figure 5.8 shows the results of spall testing for the neat PU as a function of strain rate. Figures 5.7 and 5.9 show a selection of PDV spectrograms obtained from the spall experiments of the neat samples.

Figure 5.7 (a) depicts the spectrogram of a neat PU sample impacted at $431 \mathrm{~m} / \mathrm{s}$, showing a mostly "square" wave profile, in the sense that the unloading begins about $1.5 \mu \mathrm{s}$ after the arrival of the incident shockwave. It is interesting to note the ramping free surface velocity between the arrival of the shockwave $(\mathrm{t} \approx 5.5 \mu \mathrm{s})$ and rarefaction fan $(\mathrm{t} \approx 7 \mu \mathrm{s})$. This inclined plateau is repeatable and has been seen in other samples. It is speculated that 


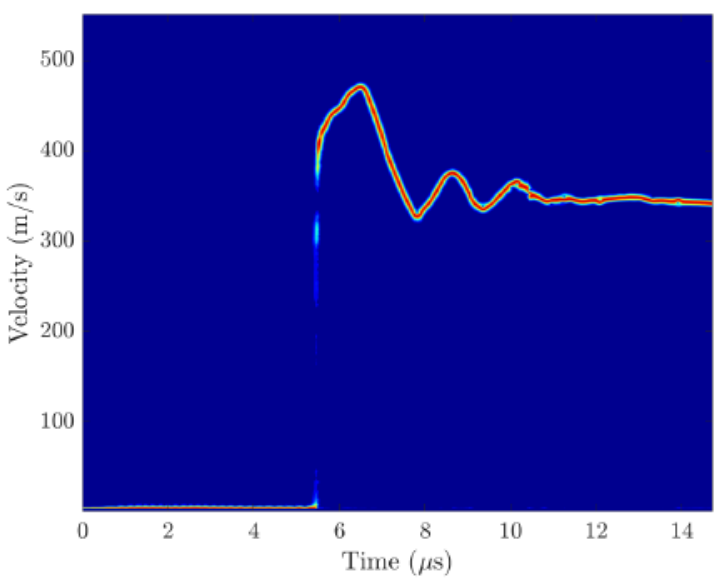

(a)

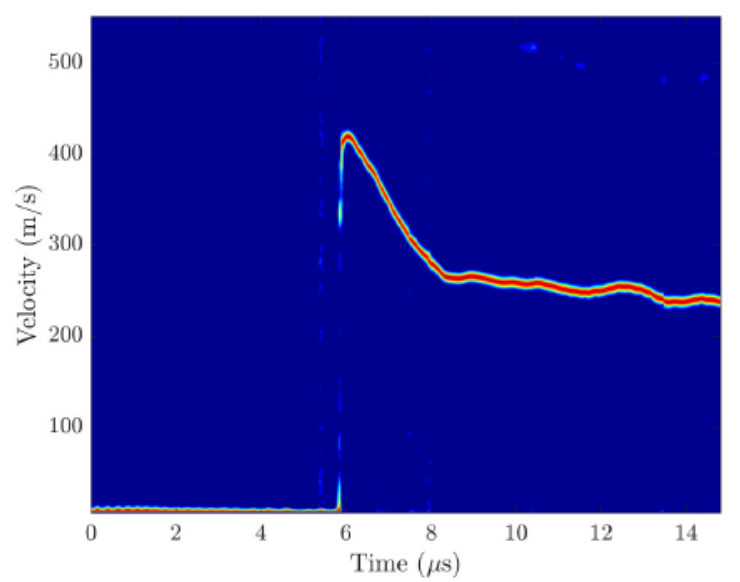

(b)

Figure 5.7: Back face velocity spectrograms obtained (a) Neat 1 and (b) Neat 3

the tilted plateau is a consequence of a visco-elastic response in the material. Rounding and tilt in the plateau has been seen in estane [29] and polycarbonate [30]. It has been explained that the visco-elastic behaviour is responsible for the "sharp rise followed by the slower approach to maximum amplitude" seen in the free surface velocity spectrogram and is often seen in polymers [30].

Figure 5.7 (b) depicts the spectrogram from a neat polyurethane sample impacted with an aluminum flyer. Here a triangular wave profile is readily observed. While triangular profiles are typically associated with higher spall strengths in literature $[27,28]$ the spall strength determined lay within the range of spall strengths determined for neat polyurethane. This may be due to the influences of competing strain rate mechanisms and loading profile mechanisms. This sample saw the lowest strain rate of the neat PU samples. Lower strain rates are typically associated with lower spall strengths $[11,13]$ while a triangular loading profile is expected to increase spall strength. As such, these two mechanisms may be negating each other to some extent, and are responsible for the minimal change in strength compared to the other neat samples. It is also interesting to note the reduced spall pulse seen in Figure 5.7 (b). A reduced spall pulse resulting from triangular loading has 
been attributed to a wider area of damage dispersing of the shock generated at the spall plane [19].

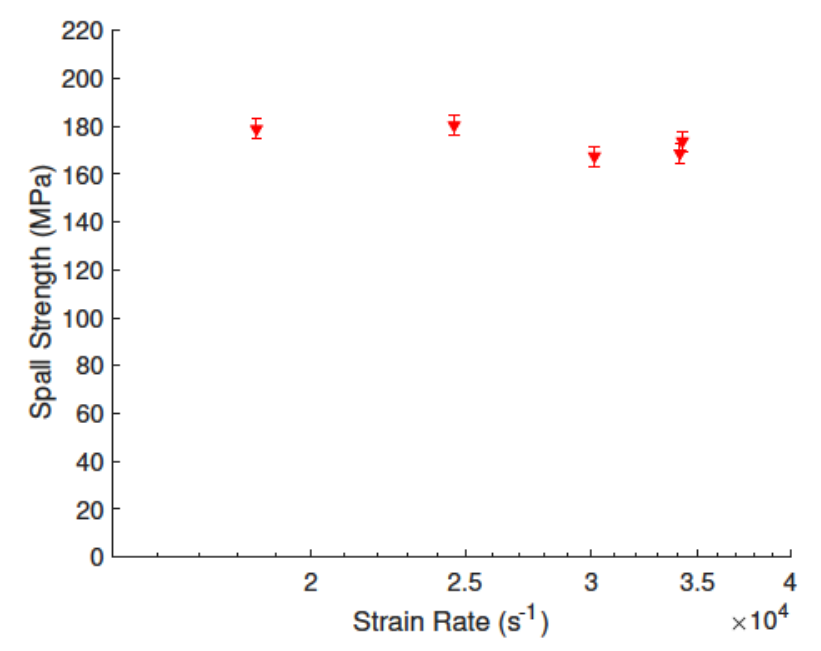

Figure 5.8: Experimental spall strength of the neat polyurethane as a function of strain rate

Having discussed wave profiles, and the fact that no change in spall strength was observed with a changing profile, it is time to consider the overall spall strength of the PU. In this study, the neat PU was found to possess a spall strength between 167.4 and $180.4 \mathrm{MPa}$, for strain rates between $1.84 \times 10^{4} \mathrm{~s}^{-1}$ and $3.4 \times 10^{4} \mathrm{~s}^{-1}$. Comparing these findings directly to other polyurethane studies is somewhat difficult, as spall in polymers is more rarely studied [29]. However other polymers can be found, such as studies in estane $\left(\sigma_{\text {spall }} \approx 130\right.$ MPa) [29] and elastomeric polyurea ( $\sigma_{\text {spall }} \approx 106 \mathrm{MPa}$ ) [75]. Considering the elastomeric nature of these two materials, it is unsurprising that the reported spall strengths are lower than those reported here for the neat PU.

Beyond elastomers, spall studies in other polymers like polycarbonate $[11,30]$ and PMMA [32] exist. Spall in polycarbonate [11,30] observed brittle fracture and spall strengths between 164 and $183 \mathrm{MPa}$, while the PMMA study reported strengths between 150 and $200 \mathrm{MPa}$ [32]. Strengths found for both the polycarbonate and PMMA are very 
comparable to the strengths presented here for the neat PU, providing some degree of validation to the results presented here.

Furthermore, an increase in the strength of PMMA by approximately one order of magnitude was observed between QS and spall experiments [32]. This increase was attributed to the Ree-Eyring theory, which suggests a strengthening response due to restricted molecular mobility at high strain rates [32]. A similar response can be seen here in the difference between the published QS strength of $17 \mathrm{MPa}$ for ClearFlex 95 compared to the observed spall strengths between 167.4 and 180.4 MPa; an increase of almost exactly one order of magnitude.

Beyond the QS response, PMMA shows minimal variation in spall strength with changing strain rate [76]. A similar trend in the neat PU is shown in Figure 5.8 with no significant variation in spall strength for different strain rates. Furthermore, as shown in Figure 5.9, the spectrograms of neat PU samples subjected to lower and higher strain rates show a similar response overall, despite the differing strain rates.

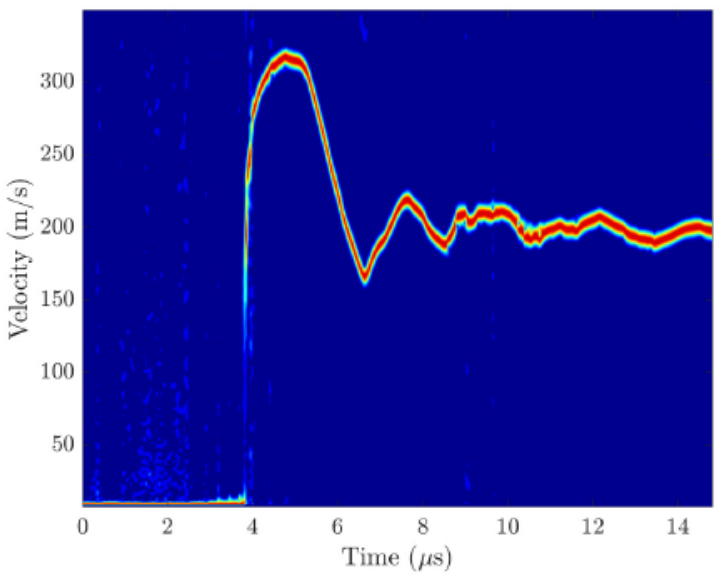

(a)

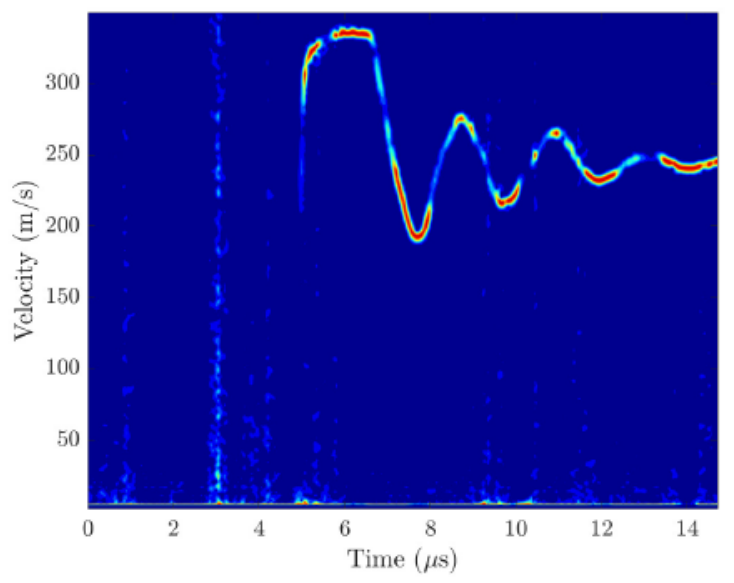

(b)

Figure 5.9: Back face velocity spectrograms obtained from two samples at two strain rates: (a) Neat 2 at $2.5 \times 10^{4} s^{-1}$ and (b) Neat 4 at $3.4 \times 10^{4} s^{-1}$

In summary, this study found the neat polyurethane possessed a strain rate insensitive 
spall strength, between 167.4 and 180.4 MPa. As discussed, an increase in strength by one order of magnitude between the published QS strength and spall strength was observed. Comparable spall strengths and increases in strength were seen in other polymers.

\subsubsection{Effect of Particle Reinforcement on Spall Strength}

In assessing the spall strength of particle reinforced polyurethane composites the effect of integrating the alumina particles into the polyurethane will be discussed first. Section 5.2.1 saw that the addition of ceramic particles resulted in an increased density, marginally reduced sound speed, and an increase in estimated Young's modulus. Regarding spall strength, the addition of alumina particles into PU results in no discernible change in overall spall strength. The experimentally determined spall strengths are presented below in Figure 5.10 for the neat, spherical alumina and irregular alumina reinforced samples.

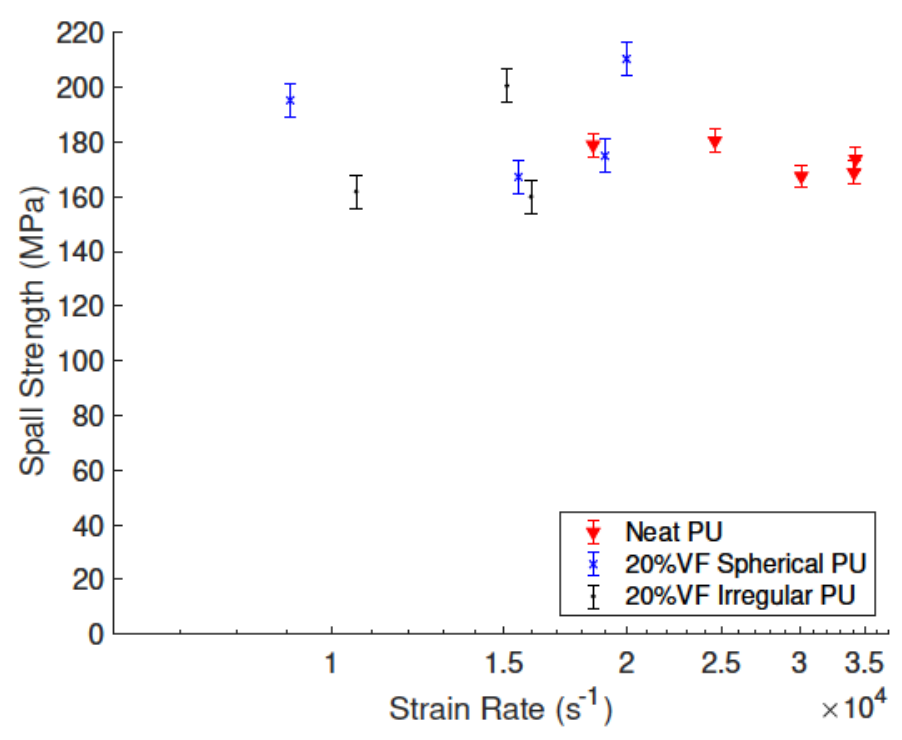

Figure 5.10: Experimental spall strength measurements plotted against strain rate of the alumina reinforced PU composites showing the effect of second phase particles

From Figure 5.10 it can be seen that the alumina reinforced polyurethane samples show a much larger scatter in spall strength. It is suspected that this scatter may be a result of 
increased density in the alumina reinforced samples. Increased sample density would yield a larger variation in spall strength for a given change in pullback velocity $\left(\Delta u_{f s}\right)$.

As discussed previously, the presence of second phase particles in a material has been observed to typically reduce the overall spall strength of the material, a finding observed in both metals $[5,17]$ and polymers $[18,19]$. This weakening is typically attributed to the matrix-filler interface behaving as a preferential site for void nucleation, for which poor matrix-filler adhesion $[18,19]$ and matrix-filler de-bonding [17] are commonly blamed.

Thus, it is suggested that the integration of alumina particles into the polyurethane saw a marginal change in spall strength because of an overall strong matrix-filler adhesion between the PU and pristine alumina. Good matrix-filler adhesion in the untreated PDMS composite put forward as the reason for the comparable strength seen in the untreated PDMS composite and the PDMS composite treated for improved adhesion. As such, strong matrix-filler adhesion may once again be responsible for the lack of change in spall strength observed between the neat PU, and the alumina reinforced PU composites. Strong matrixfiller adhesion would likely suppress the preferential nucleation of voids at the matrix-filler interface, which would otherwise be expected to result in a reduced spall strength with the addition of the alumina into the PU.

\subsubsection{Effect of Particle Morphology on Spall Strength}

Having discussed the consequences of integrating a filler into the neat polyurethane, the effect of filler morphology on the overall behaviour will be discussed. Like earlier Sections, this will begin with a review of a selection of spectrograms of interesting findings, before moving onto the the results as a whole. As such, Figure 5.11 presents one free surface velocity spectrogram from each of the spherical and irregular alumina data sets.

It is interesting to note that in the case of Figures 5.11 (a) and (b) both sample types 


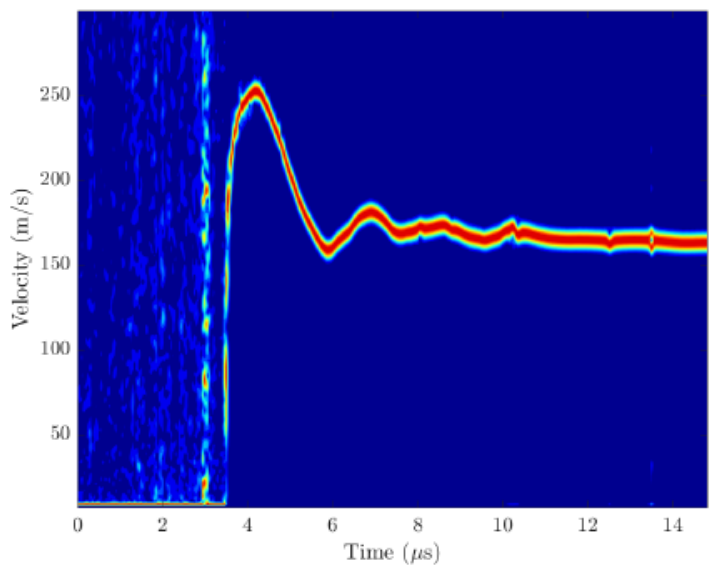

(a)

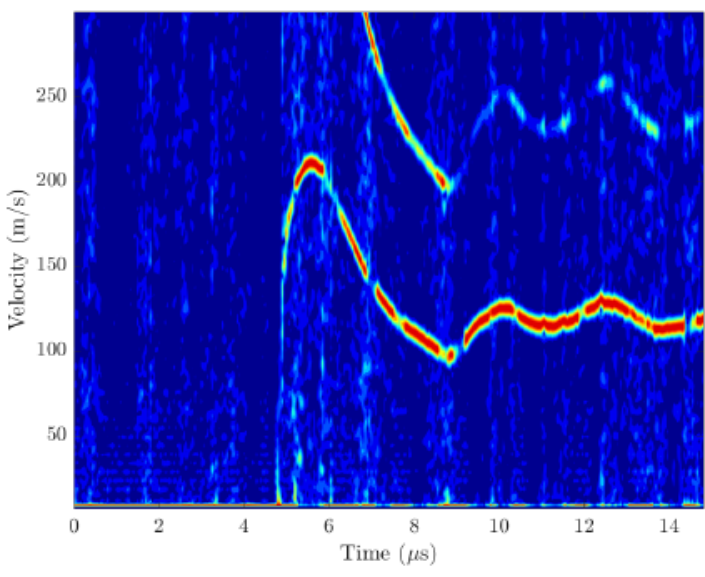

(b)

Figure 5.11: Back face velocity spectrograms obtained from samples hit at $\sim 300 \mathrm{~m} / \mathrm{s}$ with an 1/8" acrylic plate: (a) Spherical 2 and (b) Irregular 2

show an overall comparable response in the free surface velocity beginning with arrival of the incident wave, a slight rounding off prior to the peak, followed by the arrival of the unloading wave. However, it can be seen in Figures 5.11 (a) and (b) that the peak velocity obtained by Irregular 2 is about $50 \mathrm{~m} / \mathrm{s}$ less than that of Spherical 2, despite the near identical loading conditions and experimental conditions. This same response can be seen in Spherical $1\left(u_{\text {peak }}=417 \mathrm{~m} / \mathrm{s}\right)$ and Irregular $1\left(u_{\text {peak }}=367 \mathrm{~m} / \mathrm{s}\right)$; samples, which were impacted at a higher striking velocity. This suggests that the irregular alumina samples may possess a higher shock impedance compared to the spherical alumina samples. Further experiments would be required to further validate this, as the remaining spherical and irregular shots were subjected to differing loading conditions.

As seen in Figure 5.10, the range of experimentally determined spall strengths for both the spherical and irregular composite overlap. This suggests that within experimental uncertainty there is a minimal effect of particle morphology on the spall strength of alumina reinforced polyurethane. As discussed before, it was anticipated that the composites with irregular shaped filler would out-perform the ones with spherical filler, as has been seen in 
dynamic fracture experiments [42], as well as in the QS and ballistic regimes [41]. In those studies, filler with higher aspect ratios were observed to outperform comparable filler with a lower aspect ratio.

In the QS regime better performance from the irregular filler when compared to the spherical filler has been attributed to an increased influence of the filler on the polymer matrix. Further, it was speculated that irregular filler aligned with the applied tension would better transfer the applied loads. In ballistic testing, irregular particles were believed to better resist displacement and cause further strain hardening [41]. In dynamic fracture [42], larger improvements in fracture toughness with higher aspect ratio filler was attributed to improved energy dissipation with differing failure mechanisms. Low aspect ratio filler was more likely to be circumvented by the crack front, while the higher aspect ratio filler either helped dissipate energy through crack bridging or filler fracture [42].

High aspect ratio filler was found to improve performance through increased load transfer, displacement resistance/strain hardening, and increased energy dissipation. In the case of the irregular and spherical filler presented here it is speculated that failure in both sample types was dominated by void nucleation at the matrix-filler interface. At the volume fractions present, both filler geometries may have presented a sufficient density of matrix-filler interfaces such that the development of spall failure wasn't limited by interface density. In this case, both composite types could be expected to fail at a comparable strength as a result of comparable void nucleation density.

Here a comparison can be drawn from spall in silicon oils [31]. The authors observed that the presence of pre-existing bubbles in silicon oil did not reduce spall strength. This was attributed to randomly formed bubbles that were considered to serve as sufficiently favourable nucleation sites. It is suspected that a similar mechanism is responsible here. Matrix-filler interfaces, known to serve as preferential nucleation sites are believed to exist in a sufficient density in both geometries. With an abundance of nucleation site candidates 
in both sample types, spall strength is likely energy limited, and thus dominated by the applied energy, which should be comparable in both samples.

If it was desired to further investigate particle morphology effects, the quantity/frequency of nucleation site candidates would have to be addressed. To lower the number of nucleation site candidates, a few avenues of attack could be undertaken: a much increased strain rate, reduced filler volume fraction, or increased filler particle size. Increased strain rate, would narrow the tensile loading zone $[11,13]$, which means for a given interface density, fewer nucleation sites would be available for development. Likewise a reduced filler volume fraction and/or increased filler particle size, would reduce the cumulative matrixfiller interface area, thus reducing interface density. If these changes sufficiently altered the number of nucleation site candidates available, a difference in spall strength as a function of filler morphology may be observed. This can be summarized by stating, it is believed no change in spall strength was seen with varying particle geometry, due to a sufficient density of nucleation site candidates in both composites. However, a difference may be observed by varying nucleation site density with lower filler volume fractions, higher strain rates, or larger filler particles.

\subsubsection{Interpretation of Spall Strength in Negatively Treated Samples}

Prior to presenting the effect of interface adhesion on spall strength, the interpretation of the minimum free surface velocity needs to be discussed. Spall experiments in silanetreated PU samples tended to show an unloading process consisting of an inflection point, followed by a later minimum seen several microseconds later. The two minimum velocity candidates are shown in Figure 5.12. The minimum value was taken to be the inflection point after considering strain rate, internal wave interactions, and experimental timing.

Evaluating the strain rate for both phases of the pullback shows a strain rate on the 


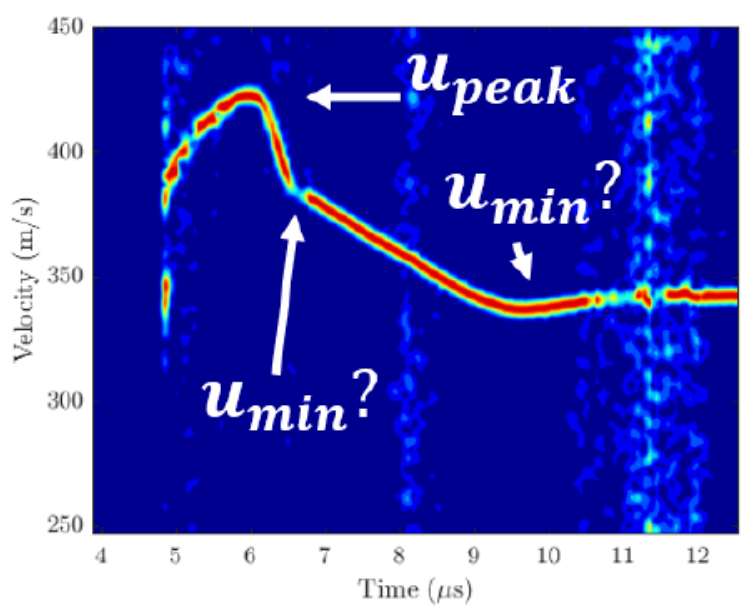

Figure 5.12: Free surface velocity spectrogram of a silane treated polyurethane sample showing the two candidates for minimum particle velocity

order of $10^{4} s^{-1}$ for the initial pullback, compared to a strain rate on the order of $10^{3} s^{-1}$ for the region after the inflection point. Experiments performed on the other sample types saw strain rates on the order of $10^{4} s^{-1}$. Considering the fact that the negatively treated samples were thinner than the other sample types and lacked the acrylic striker plate, it is expected that the thinner silane-treated samples would present a higher strain rate. As such, this suggests that taking the inflection point as the minimum is the more reasonable of the two options. Furthermore, looking at the free surface velocity traces of spall in other elastomers [11] shown in Figure 5.13, a decrease in the free surface velocity slope $(\dot{u})$ can be seen after the onset of spall, indicating a reduction in strain rate. This same change in strain rate can be seen in the PDV traces of the PDMS samples subjected to spall presented in Section 5.1. A decrease in $\dot{u}$ can be seen to occur after the inflection point of the velocity spectrogram presented in Figure 5.12.

Before moving on to other discussion points, the lack of a distinct spall pulse should be addressed. While the spall traces presented in Figure 5.13 show a clear and distinct uptick in particle velocity after spall, the lack of this pulse in the study presented here can 


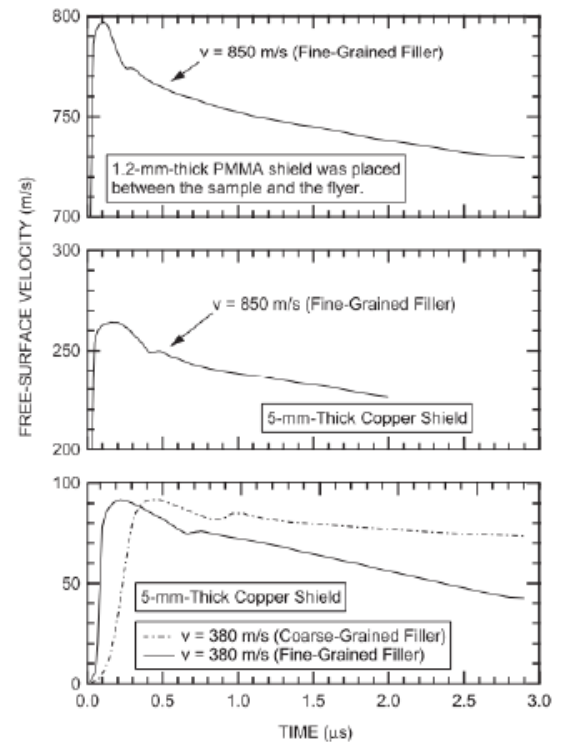

(a)

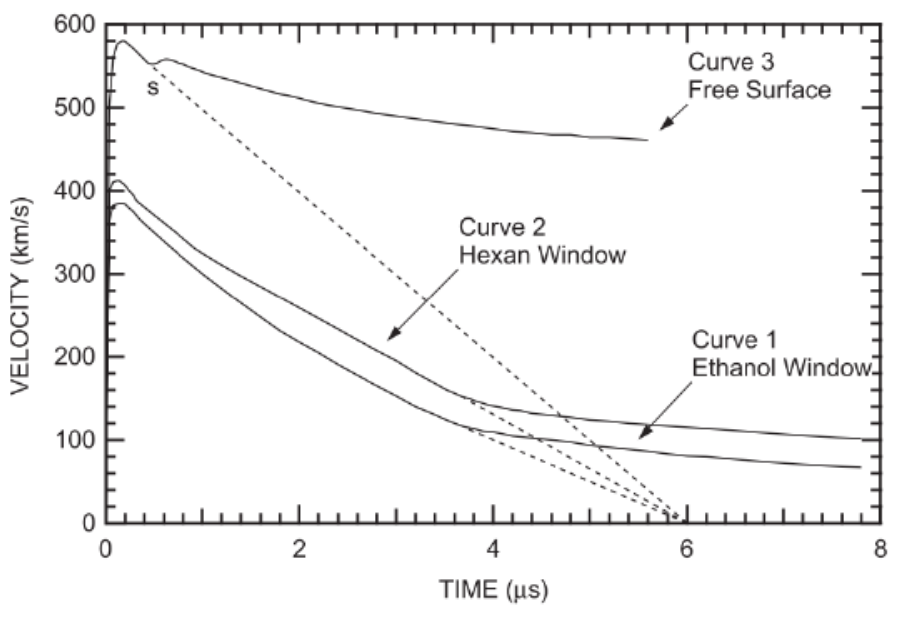

(b)

Figure 5.13: (a) Spall traces of particle filled elastomeric rocket propellant simulants (b) and rubber [11]

be attributed to the attenuation of the RG shockwave generated at the spall plane. In the spall testing of CNT reinforced epoxy composites [19], a reduced spall pulse amplitude was attributed to internal dispersion of the wave due to damage or plastic deformation resulting from the induced tensile state.

Moving to the time line of the experiment, one should consider the amount of time taken for the negative samples to unload and spall. Modelling the x-t diagram in Figure 5.14 of a negative sample struck at $400 \mathrm{~m} / \mathrm{s}$ one can see that the back face velocity should expect a plateau duration of approximately $1.5 \mu \mathrm{s}$, prior to the arrival of the leading edge of the $\mathrm{RG}$ rarefaction fan, with the tailing edge of the fan arriving about $1 \mu$ s after that. Since spall relies on the interaction of rarefaction fans to generate a tensile state exceeding the strength of the material in question, it can be concluded that spall would occur prior to the arrival of the tailing edge of the RG fan. The above described inflection point lies within this one microsecond window, while the minimum point of the free surface spectrogram is 


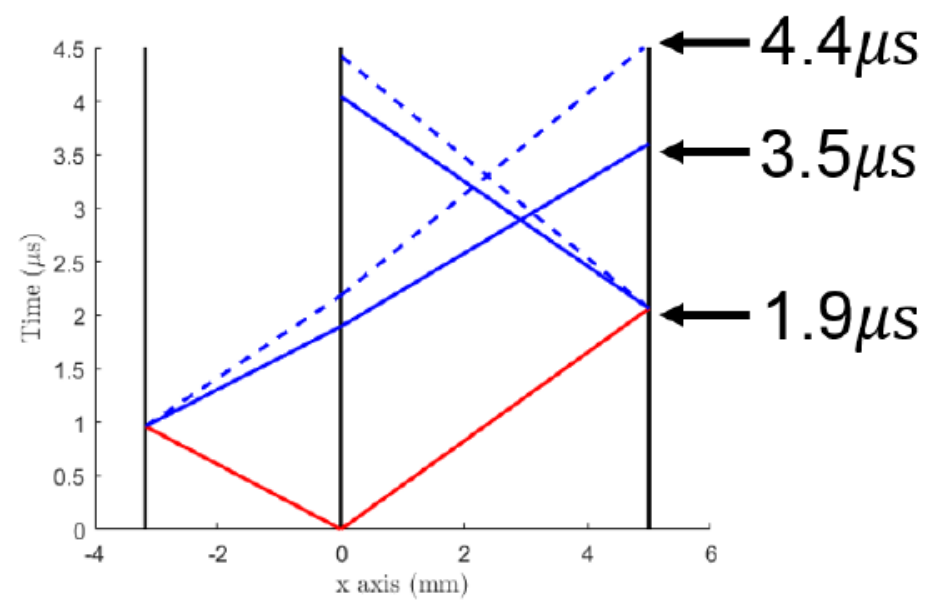

Figure 5.14: $x-t$ diagram of spall in a negatively treated sample struck at $400 \mathrm{~m} / \mathrm{s}$

seen almost $4 \mu \mathrm{s}$ after the onset of unloading (seen at $\sim 9.5 \mu \mathrm{s}$ in Figure 5.12).

Lastly, it is interesting to note that a study of spall in elastomeric polyurethane (estane) [29] observed a minimal spall pulse at the point taken to be the minimum particle velocity. A sharp reduction in $\dot{u}$ was observed after this point. In summary, the spall strength of the silane-treated polyurethane samples has been interpreted using the "inflection point" in the free surface velocity spectrogram after considering strain rate, internal wave interactions, experimental timing, and comparable literature.

\subsubsection{Interface Adhesion Effects on Spall Strength}

In reviewing the spall strengths listed in Table A.1 and shown in Figure 5.15, it can be seen that impeding the interface adhesion between the alumina and PU results in a drastic reduction in strength. The significant reduction in strength as a result of impeding adhesion in the PU composites proves interesting as a comparable reduction in strength was also seen in the PDMS study as a consequence of impeding adhesion. As discussed earlier, interfaces play an important role in dictating the spall strength of a composites, due to the tendency of interfaces to serve as preferential void nucleation sites $[5,17]$. Furthermore, the results 
of the PDMS study suggests an inherently active surface present on the untreated alumina, which is likely bonding to the PU matrix. Comparing the active surface of the pristine alumina to the alumina treated to suppress this bonding, it is straightforward to see how the silane treatment plays a role in reducing the overall spall strength of the composite.

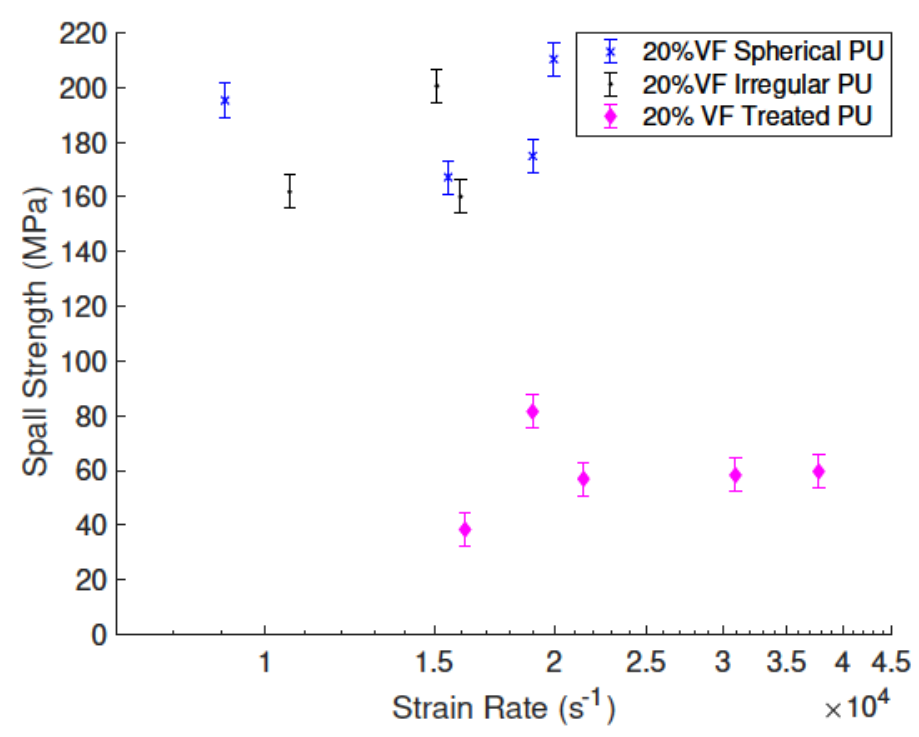

Figure 5.15: Experimental spall strength measurements plotted against strain rate of the alumina reinforced PU composites showing the effect of impeded interface adhesion

A stronger interface adhesion in the untreated composite benefits the overall composite by behaving as a less suitable candidate for spall nucleation. As per the energy-limited and flaw-limited theories [15,22], this would suggest that more energy is expended in developing the interface, so that it can behave as a suitable nucleation site, resulting in an increased spall strength. On the contrary, the silane-treated composite is likely to behave as an excellent candidate site for spall nucleation, as the low strength interface would possess little strength to resist void nucleation.

Beyond directly measuring spall strength, some further points of interest were seen in the plate impacts of silane-treated samples. Looking at Table A.1, it can be seen that all silane-treated samples were impacted at a minimum flyer velocity of approximately 
$400 \mathrm{~m} / \mathrm{s}$. This was due to the fact that silane samples impacted at a lower velocity $(300 \mathrm{~m} / \mathrm{s})$ remained whole, with superficial external damage. Recovery and sectioning of one of these samples (Figure 5.16) impacted at $300 \mathrm{~m} / \mathrm{s}$ observed the absence of a defined spall plane.

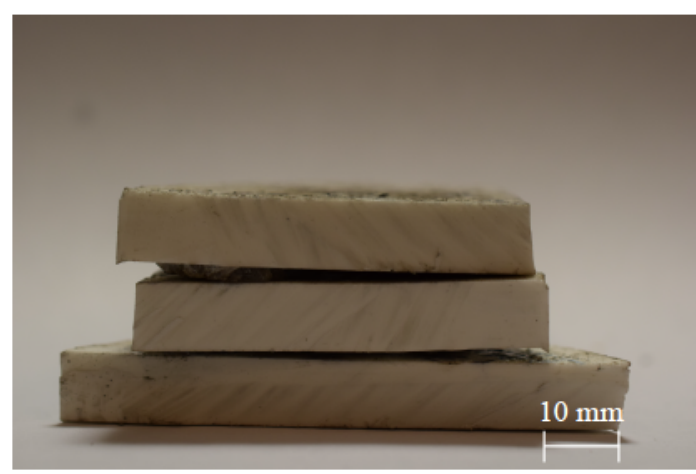

(a)

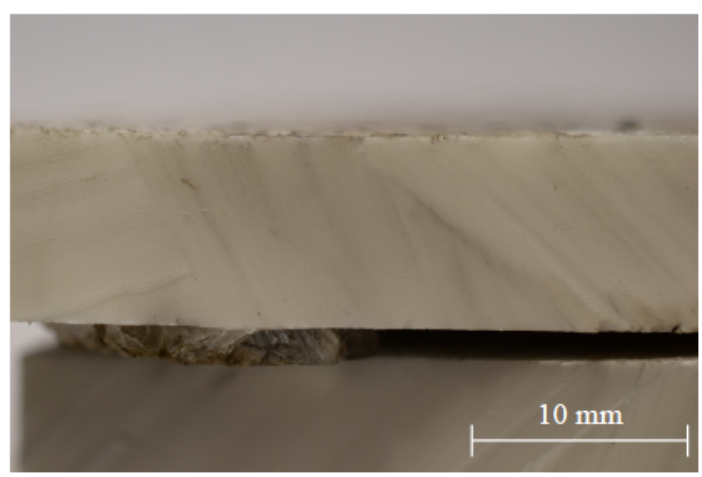

(b)

Figure 5.16: (a) Cross-sectional photo of silane-treated, 20\% alumina-reinforced PU sample showing an absence of a clear spall plane. (b) Zoomed image to to show a magnified portion of the cross-section presented in (a)

The absence of a spall plane in these samples is somewhat unsurprising as similar behaviour has been seen in other rubbers, where samples showed minimal damage [43]. Furthermore, upon sectioning of those recovered samples, no spall plane was observed in the sample even though the applied tensile load exceeded the measured spall strengths. As discussed previously, regarding elastomers [11], the "spall pulse" in elastomers has been taken to correlate to the nucleation of voids in the material rather than the strength of the material prior to complete failure, which explains the absence of a clearly defined spall plane. On the contrary, impacts of the silane treated samples at a higher velocity saw the formation of a clearly defined spall plane (Figure 5.17 (a)). The PDV spectrogram corresponding to this spalled sample is shown in Figure 5.17 (b).

As discussed previously, and seen in Figure 5.17 (a) the geometry of these experiments slightly differed from the ones described above in Chapter 4 regarding the experimental 


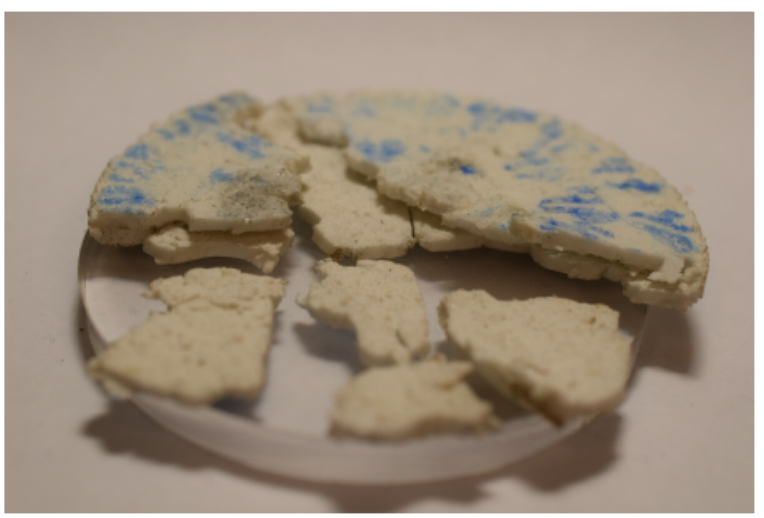

(a)

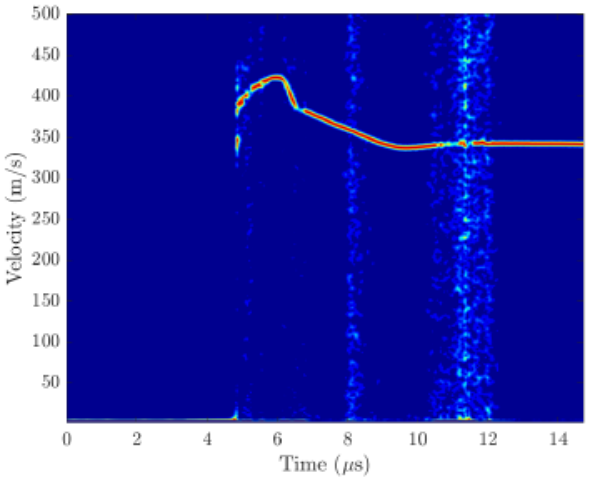

(b)

Figure 5.17: (a) Clear spall plane formation in a silane-treated, 20\% alumina-reinforced PU sample. (b) PDV spectrogram of the sample in (a) during the spall experiment

setup. Apart from shot "Negative 5" the silane-treated samples were made thinner and shot without the acrylic striker plate, in order to mitigate any concerns of wave attenuation that may have been preventing the clear observation of spall in the samples. "Neat 4 " and "Neat 5" were tested with this reduced thickness to ensure that a changing thickness did not detrimentally affect the results of the study. It appears that the change in thickness did not affect the experimental results, apart from a moderate increase in the strain rate, which is an expected consequence of using a thinner target. As expected, the RG rarefaction fan from the flyer backface spread out less prior to reaching the backface of the target, due to the shorter elapsed travel distance.

Once again, it should be pointed out that other than the inclusion of the silane, the PU composite treated for impeded adhesion was essentially identical to the untreated PU composite, with the same volume fractions, same filler material/geometry, and same PU matrix. This supports the conclusion that differences seen in the spall strength of the two composites are likely a direct consequence of impeded adhesion. To further support this theory, the influence of the silane on the PU itself was directly investigated and will be discussed in Section 5.2.7. 


\subsubsection{Silane Polyurethane Interactions}

In addition to the shape and interface adhesion effect studies presented above, there was some concern that the silane itself may have been interfering with the polymerization of the polyurethane. If this was the case, a degradation in the composite strength may not have been a result of impeded interface adhesion, but rather a compromised matrix strength. This concern was addressed by creating a new sample type consisting of a polyurethane-silane blend, in the ratio seen in the negatively treated composites. In essence, a blend was created equivalent to one where the alumina was removed from the silane-treated composite. These samples were then measured for density, sound speed, and shot under the same conditions as the neat polyurethane. As can be seen in Table 5.5 and Figure 5.18 a negligible difference was seen in the spall strength and physical properties of the neat PU and PU+silane blend.

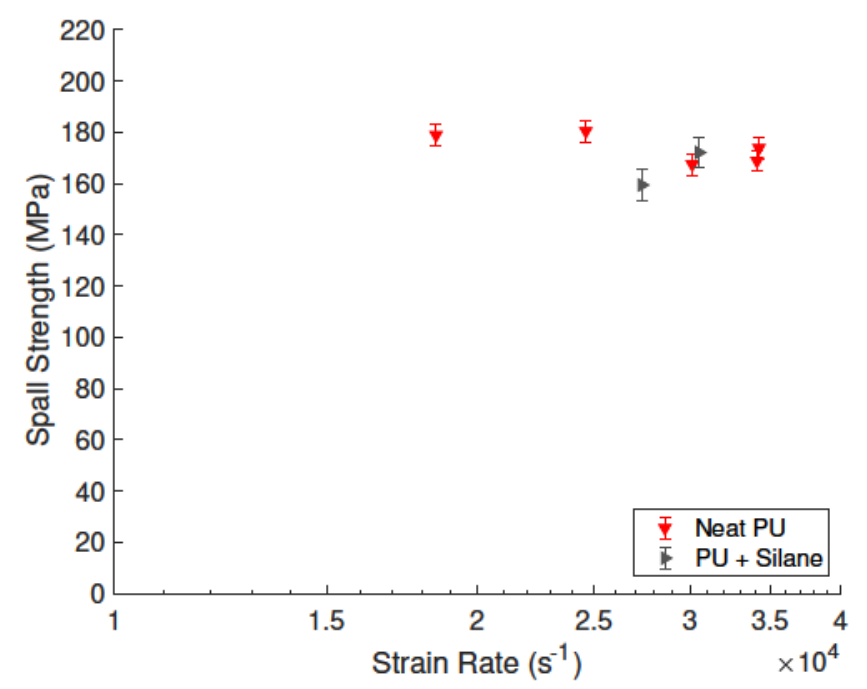

Figure 5.18: Experimental spall strength measurements of the PU and PU+silane blend plotted against strain rate

This implies the silane alone has a minimal effect on the overall chemistry of the polyurethane, suggesting that differences seen in the composite treated for negative adhesion can be attributed to impeded interface adhesion. Furthermore, the PDV traces of the 


\begin{tabular}{|c|c|c|}
\hline Material & Neat PU & PU + Silane \\
\hline Mean Spall Strength $(\mathrm{MPa})$ & 174 & 165 \\
\hline Measured Sound Speed $(\mathrm{km} / \mathrm{s})$ & $2.17 \pm 0.01$ & $2.02 \pm 0.01$ \\
\hline Measured Density $\left(\mathrm{g} / \mathrm{cm}^{3}\right)$ & $1.09 \pm 0.03$ & $1.09 \pm 0.03$ \\
\hline
\end{tabular}

Table 5.5: Comparison of the properties of neat polyurethane and a polyurethane silane blend

neat PU and PU+silane blend shown in Figure 5.19 are very similar, further eliminating the idea that the silane did impede the polymerization of the PU.

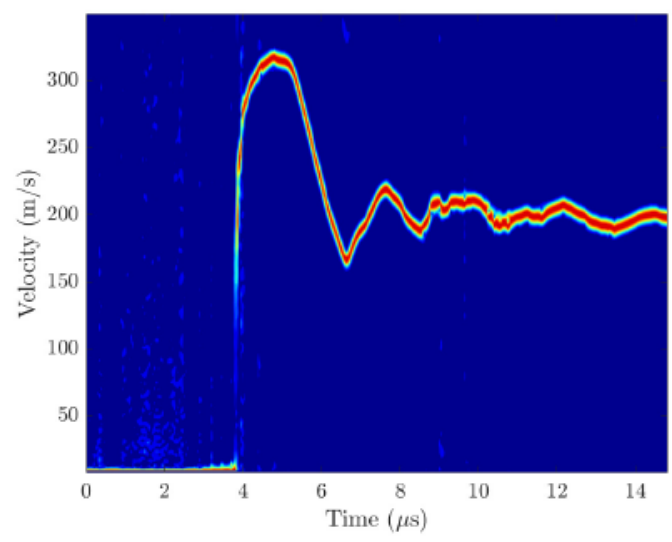

(a)

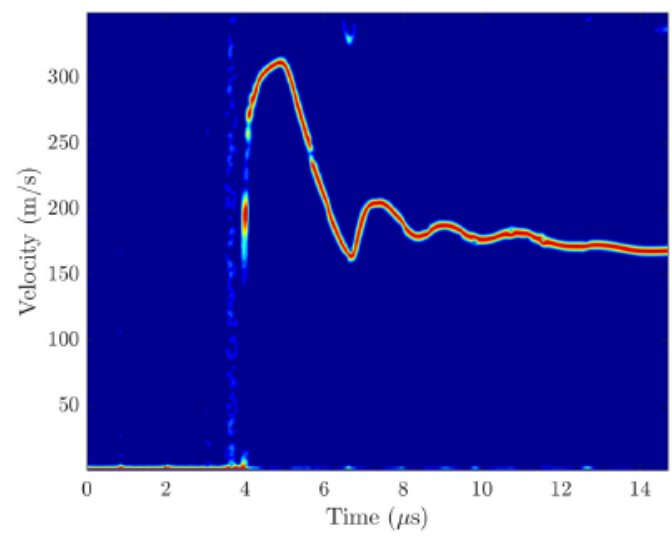

(b)

Figure 5.19: (a) Neat polyurethane target struck by an acrylic flyer at $290 \mathrm{~m} / \mathrm{s}$ (b) polyurethane silane blend target struck by an acrylic flyer at $292 \mathrm{~m} / \mathrm{s}$

A comparison of the recovered spall samples did reveal some interesting findings. As was shown in Figure 4.8, the neat PU shows far more damage under spall testing, while the PU+silane blend (Figure 5.20) shows minimal damage by comparison. The neat polyurethane can be seen to have clearly spalled, while the PU+silane blend remained whole, with minor damage to the external surfaces. Due to the transparent nature of the PU+silane blend, some small internal cracking can be seen, presenting itself as a faint, planar network of small white lines, within the sample. This may be a result of the silane 
driving the PU to behave more like an elastomer. In other research [11], elastomers have been seen to present minimal damage, even when subjected to loads that well exceed the reasonable strengths of the material.

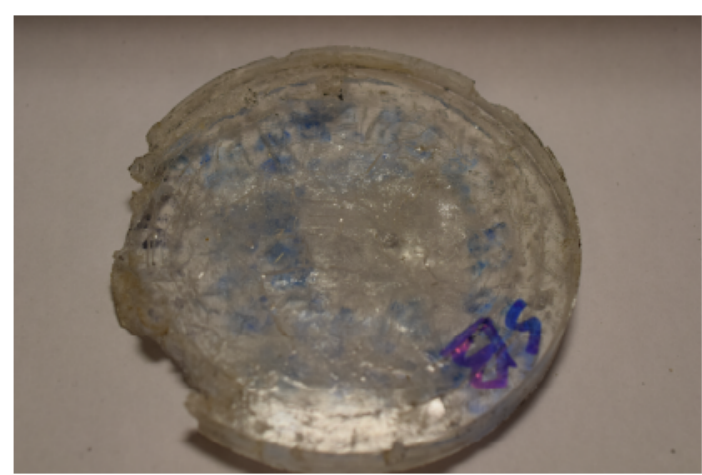

(a)

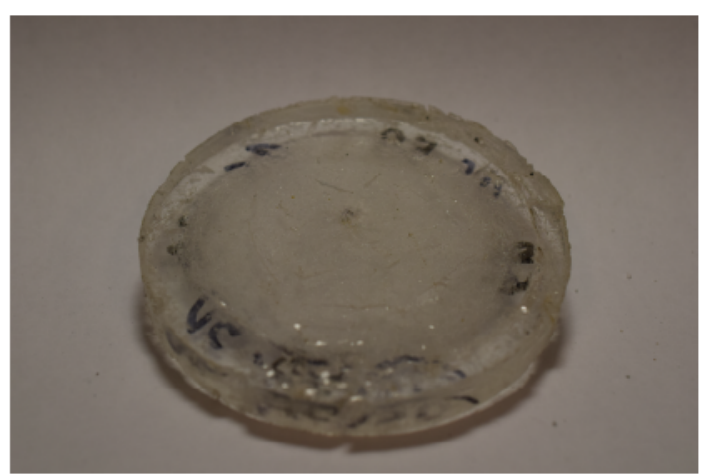

(b)

Figure 5.20: Images of recovered PU silane spall samples: (a) $1 / 8$ " acrylic impactor at 281 $\mathrm{m} / \mathrm{s}$, (b) $1 / 8$ " aluminum impactor at $290 \mathrm{~m} / \mathrm{s}$

As discussed here, while a spall plane was still observed in the samples, recovered samples showed less damage overall, compared to the neat polyurethane; a behaviour that was attribute to a more elastomeric behaviour in the blend. The silane+polyurethane blend has been seen to perform quite similarly to the neat polyurethane samples, showing a comparable density, sound speed and spall strength. In summary, experimental results suggest that the silane itself did not induce any adverse effects in the polyurethane, supporting the conclusion that impeded adhesion was responsible for the reduction in spall strength, seen in the previous Section. 


\subsubsection{Fracture Surface Analysis}

During the testing of the PU composites, samples were retrieved after each shot for qualitative analysis of the fracture surface. Further analysis was performed using the scanning electron microscopy (SEM) facility in the Nano-imaging facility at Carleton University. The complete collection of these SEM images are presented in Appendix C. The images presented here show the fracture surfaces, with the line of site normal to the fracture plane. Shown in Figures 5.21, 5.22, and 5.23 are a selection of these images.

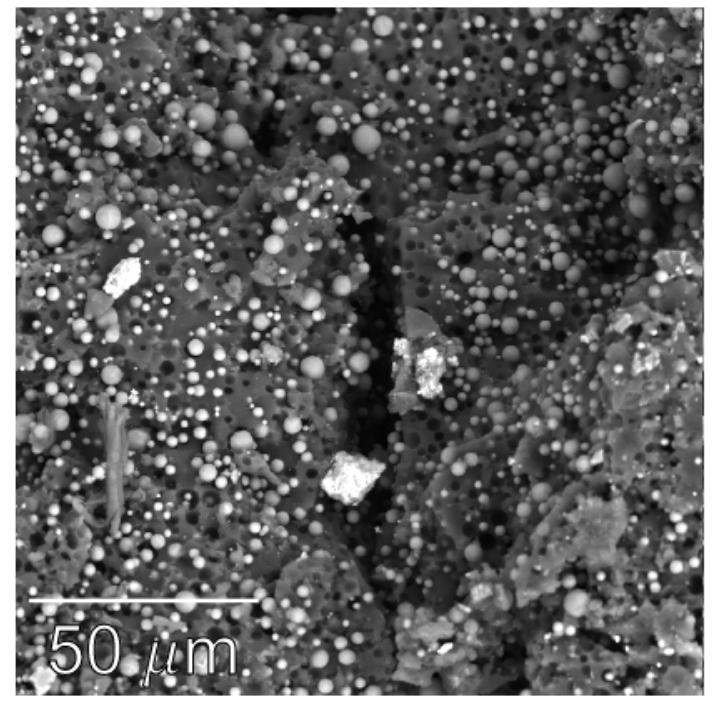

(a)

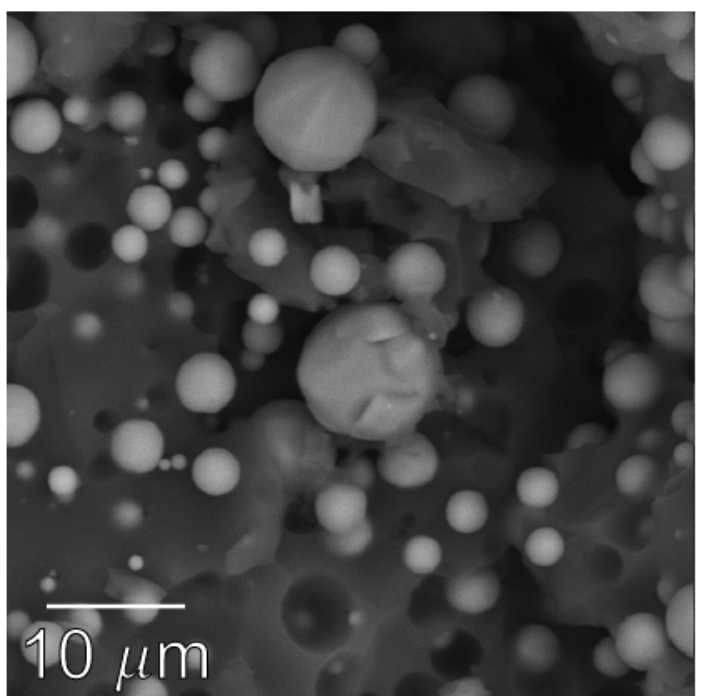

(b)

Figure 5.21: A selection of SEM images displaying the fracture surface of a spherical alumina reinforced PU sample

As discussed previously, it has been observed in past work that the matrix-filler interface behaves as a site for the preferential nucleation of voids, leading to the pullout of filler from the matrix. As can be seen in Figure 5.21, this appears to once again be the case, with a number of pores showing where the spherical alumina was pulled out of the matrix under the applied dynamic tensile load. Pores suggesting pullout of the silane-treated spherical filler can also be seen in Figure 5.23. This supports the conclusions drawn earlier, 
highlighting the fact that the matrix-filler interface plays a large role in the failure of these composites under dynamic tensile load.

Likewise, Figures 5.21 and 5.23 also appear to display no signs of filler fracture. The filler visible in the images appears whole and spherical. The angular, white clumps visible in Figure 5.21 were analysed by the nano-imaging facility to be calcium based, rather than aluminum based. The contamination is likely a consequence of the retrieved sample ricocheting inside the catch tank after impact. The lack of apparent fracture in the filler indicates that the spall plane developed through the growth and coalescence of voids in the PU matrix.

Possible fracture of the irregular shaped filler in Figure 5.22 is more difficult to assess due to the inherent shape of the filler. Fractured irregular filler may be easily mistaken for unfractured filler. Likewise, pullout of the irregular filler is not readily apparent, as the geometry of the pores left behind by pulled out filler would vary due to the inconsistent geometry of the irregular filler.

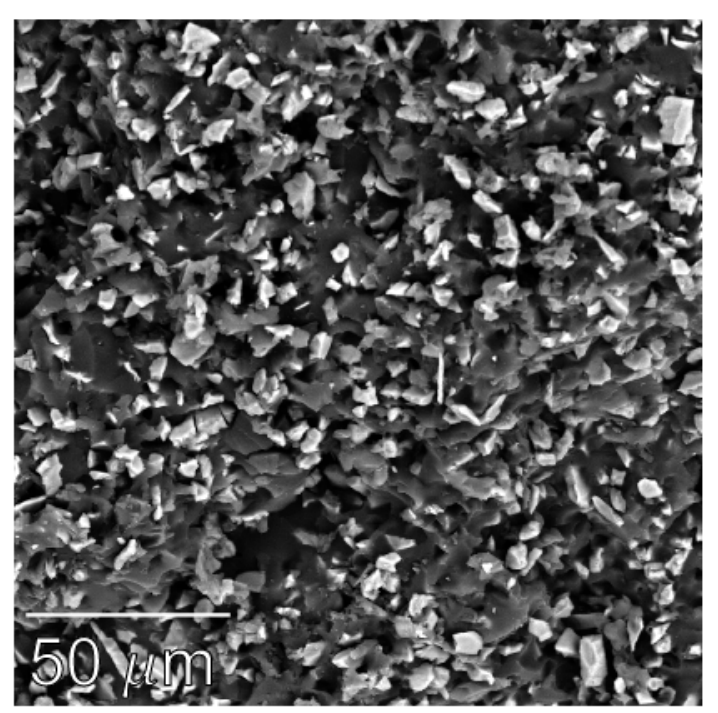

(a)

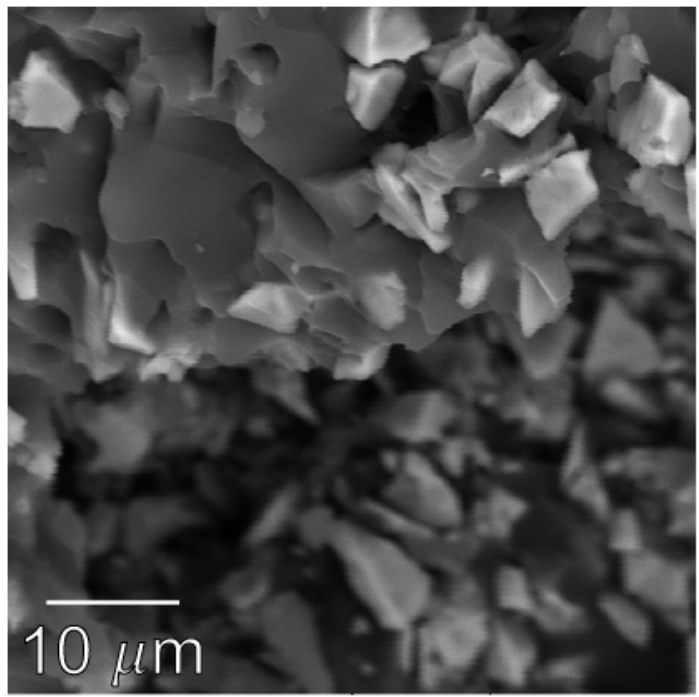

(b)

Figure 5.22: A selection of SEM images displaying the fracture surface of an irregular alumina reinforced PU sample 
It is also interesting to note that the filler appears well dispersed in each of the SEM images presented here, with little sign of clumping or agglomeration. As discussed previously, poor dispersion of filler in composites has been attributed to a lower tensile strength in the QS regime $[36,37]$. These SEM images serve to alleviate concerns of poor filler dispersion, that would otherwise detrimentally influence the overall composite.

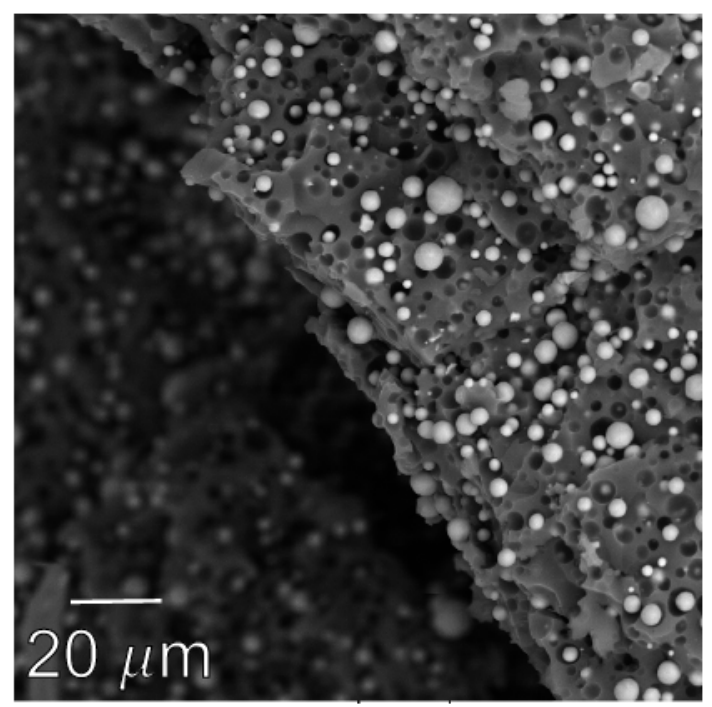

(a)

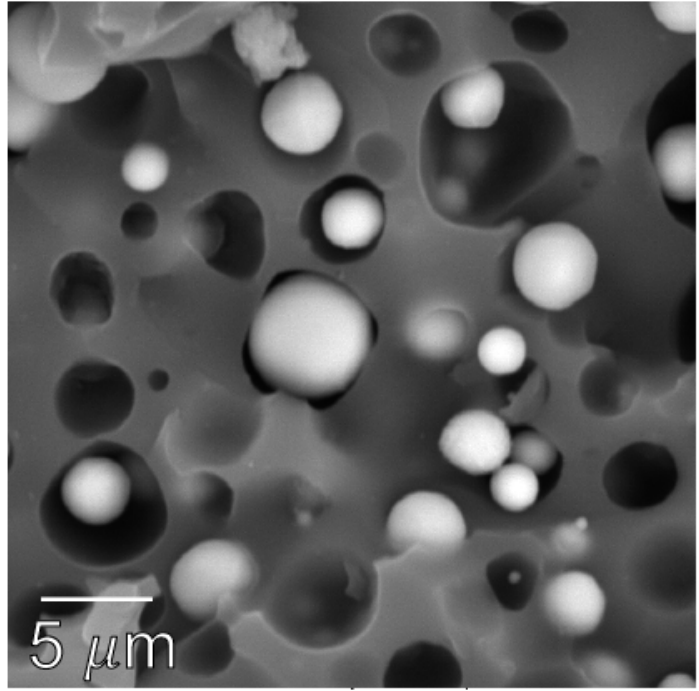

(b)

Figure 5.23: A selection of SEM images displaying the fracture surface of a silane-treated, spherical alumina reinforced PU sample

Lastly, it is interesting to note the differences between the untreated spherical sample seen in Figure 5.21 (b) and the silane treated sample shown in Figure 5.23 (b). This difference can be characterized by the matrix-filler boundaries seen in the respective images. In the case of the untreated sample, the filler appears well bonded to the matrix, with pullout of the filler resulting in round pores. The silane treated sample show gaps between the matrix and filler, and irregularly shaped pores. Gaps between the matrix and filler are considered to a consequence of the impeded matrix-filler adhesion driven by the silane treatment, and would readily as void nucleation sites in the composite. Likewise the irregularly shaped voids left behind by pulled out filler are not evident in the pristine spherical 
samples, further suggesting the presence of poor adhesion between the matrix and filler.

In summary, the suppression of matrix-filler adhesion by silane treatment has been observed under SEM to reduce bonding between the matrix and filler, and is considered responsible for the drastic reduction in spall strength observed under plate impact testing. This further supports the conclusions drawn in this study, demonstrating the influence of matrix-filler adhesion on the spall strength of multi-phase systems. 


\section{Chapter 6}

\section{Conclusion}

With matrix-filler interfaces known to behave as sites for preferential void nucleation, this thesis postulated that particle morphology and matrix-filler interface adhesion would likely serve to influence the overall spall strength in a multi-phase system. In this study, variations in filler morphology and surface chemistry have been investigated in particlereinforced PDMS and PU composites. Experimentally, these tailored composites have been evaluated under plate impact conditions to assess the influences of these filler properties on overall spall strength.

In evaluating matrix-filler adhesion, it was anticipated that spall strength would correlate with interface adhesion. Increased interface adhesion was expected to better resist void nucleation at the interface, resulting in an improved spall strength. In the PDMS study, no change in spall strength was seen with improved adhesion, a finding attributed to the inherently active surface chemistry of the untreated alumina filler. In both studies, a drastic reduction in spall strength was observed in samples where the filler was treated to impede adhesion. Reductions in strength were attributed to a loss of adhesion at the particle surface, despite otherwise identical sample composition. Concerns of silane interference with the polymer matrix were addressed by showing near identical behaviours and spall strengths between the neat polyurethane and silane-polyurethane blend.

With regards to filler morphology it was anticipated that particle morphology would 
play a role in the overall spall strength of a material due to the variation in cumulative interfacial surface area associated with a changing filler geometry. However, particle morphology was found to bear little influence on the spall strength or measured mechanical properties. Comparable spall strengths were attributed to the presence of a sufficient matrix-filler interface density in both sample types.

As stated in the introduction of this thesis, the objective of this study was to assess the roles played by particle morphology and interface adhesion, in the spall strength of particle reinforced polymer matrix composites. As discussed here, these goals have been met, through the experimental findings of the PDMS and PU studies. These results demonstrate the importance of properly accounting for the interactions between the phases of a composite, and their influence on the bulk performance of composites under dynamic loading.

Outside of the academic world, it was intended that the findings presented here, could be used to assist in the future design of flexible armours, for ejecta protection applications. This study has assisted in the pursuit of this goal, by directly investigating the influences of these phase interactions, at the interface, under strain rates relevant to ballistic loading. From this investigation, the understanding of phase interactions within multi-phase systems has stepped forward, which may allow for improved armour design without trading mobility for protection.

\subsection{Future Work}

Regarding the future of spall and plate impact research here at Carleton, a few elements present themselves as viable candidates for further pursuit. Regarding shape effect in the alumina reinforced polyurethane samples, the effects of changing strain, filler volume fraction, or filler size should be investigated. Varying these parameters could change failure from energy-limited to flaw-limited, which may yield some interesting information 
regarding particle morphology effects, and the influence of particle reinforcement on spall strength. In addition, integration of Fourier transform infrared spectroscopy (FTIR) would prove beneficial for use in directly quantifying matrix-filler adhesion. However, due to administrative difficulties at Carleton, this option was not feasible at the time of this study.

With regards to the gas gun facility, implementation of a soft recovery system for samples should be further pursued. Samples would be retrieved by providing a soft container for the post spall sample to decelerate into, in a non-destructive manner. Furthermore, a soft recovery system would prevent the ricocheting of the target around the catch tank, reducing the chance of sample contamination. Contamination of the sample may complicate identification of features SEM imaging.

A typical soft recovery system consists of a rag filled container mounted aft of the target. However, preliminary attempts at soft sample recovery using a rag filled container resulted in samples ricochetting out of the soft catch container, coupled with immediate destruction of the container. Spall recovery experiments in literature use assemblies to strip the sabot, followed by graded foams to gradually decelerate the sample, bringing the sample to rest in a ballistic foam/rag backed container [77].

Of further consideration to the recovery of samples, is the use of tapered momentum trap rings to eliminate the effect of edge release waves $[17,47]$. Edge release waves could otherwise complicate the loading history of the sample, which could influence the fracture surface in an unanticipated manner. Tapered rings address this by tapering the outer diameter of the target plate, giving it the shape of a truncated cone. An outer ring is mated to this tapered edge. Properly designed, this assembly ensures the sample maintains the uni-axial loading condition throughout the experiment [78].

Lastly, while the SEM images presented here observed the fracture surface, it is advised that viable cross-sectioning methods are pursued in future imaging endeavours. Imaging of the cross-section around the fracture surface, can provide useful information on the 
development of the spall plane. One is more likely to observe the development of voids, crack tips and other transitory features that are otherwise precursors to the primary spall plane. Possible methods for cross-sectional cutting include cryogenic cutting, which should section the sample in a non-destructive manner. 


\section{List of References}

[1] D. C. Smith, "Extremity Injury and War: A Historical Reflection," Clin. Orthop. Relat. Res., vol. 473, no. 9, pp. 2771-2776, 2015.

[2] “Annual Report," tech. rep., Joint Improvised Explosive Device Defeat Organization, Washington, DC, 2010.

[3] S. L. Eskridge, C. A. Macera, M. R. Galarneau, T. L. Holbrook, S. I. Woodruff, A. J. MacGregor, D. J. Morton, and R. A. Shaffer, "Injuries from combat explosions in Iraq: Injury type, location, and severity," Injury, vol. 43, pp. 1678-1682, oct 2012.

[4] Z. Rosenberg and E. Dekel, Terminal Ballistics. Berlin, Heidelberg: Springer Heidelberg Dordrecht London New York, 2012.

[5] G. J. Appleby-Thomas and P. J. Hazell, "A study on the strength of an armour-grade aluminum under high strain-rate loading," J. Appl. Phys., vol. 107, no. 12, pp. 1-12, 2010.

[6] Q. Ma, R. Guo, Z. Zhao, Z. Lin, and K. He, "Mechanical properties of concrete at high temperature-A review," Constr. Build. Mater, vol. 93, pp. 371-383, 2015.

[7] "Bearing investigation," in Railw. Tech. handbook, Vol. 1, chapter 6, vol. 1, pp. 122$135,2012$.

[8] P. W. Cooper, "III Shockwaves," in Cooper - Explos. Eng., ch. 14-19, pp. 167-273, New York: Wiley-VCH, 1996.

[9] A. K. M., "Computer Simulation of Spall Crack Formation," Struct. Dyn. - EURODYN, no. 1, pp. 475-477, 1999.

[10] K. T. Ramesh, "High Rates and Impact Experiments," in Springer Handb. Exp. Solid Mech., vol. 2, pp. 929-960, Boston, MA: Springer US, mar 2008.

[11] T. Antoun, L. Seaman, D. R. Curran, G. I. Kanel, S. V. Razorenov, and A. V. Utkin, Spall Fracture. New York, NY: Springer, 1st ed., 2003. 
[12] M. A. Meyers and C. Taylor Aimone, "Dynamic fracture (spalling) of metals," Prog. Mater. Sci., vol. 28, pp. 1-96, jan 1983.

[13] D. R. Jones, S. J. Fensin, D. T. Martinez, C. P. Trujillo, and G. T. Gray, "Effect of peak stress and tensile strain-rate on spall in tantalum," J. Appl. Phys., vol. 124, no. 8, 2018.

[14] H. Nahme and E. Lach, "Dynamic Behavior of High Strength Armor Steels," Le J. Phys. IV, vol. 07, pp. C3-373-C3-378, aug 1997.

[15] D. E. GRADY, "Incipient spall, crack branching, and fragmentation statistics in the spall process," Le J. Phys. Colloq., vol. 49, pp. C3-175-C3-182, sep 1988.

[16] S. J. Fensin, E. K. Walker, E. K. Cerreta, and G. T. G. Iii, "When do interfaces become important for failure?," EPJ Web Conf., vol. 94, p. 2010, 2015.

[17] C. L. Williams, T. Sano, T. R. Walter, J. Bradley, and L. J. Kecskes, "The Role of Second Phase Intermetallic Particles on the Spall Failure of 5083 Aluminum," J. Dyn. Behav. Mater, vol. 2, no. 4, pp. 476-483, 2016.

[18] J. E. Pepper, J. Huneault, M. Rahmat, and O. E. Petel, "Spall strength measurements in EPON 828 epoxy and an epoxy/carbon nanotube composite," in AIP Conf. Proc., vol. 1979, p. 070024, 2018.

[19] J. Huneault, J. E. Pepper, M. Rahmat, B. Ashrafi, and O. E. Petel, "Spall Characterization of EPON 828 Epoxy with Embedded Carbon Nanotubes," J. Dyn. Behav. Mater., vol. 5, pp. 13-23, mar 2019.

[20] C. Comtois-Arnaldo, S. Ouellet, and O. E. Petel, "A ballistic performance study on particle-infused elastomeric systems," in AIP Conf. Proc., vol. 1979, p. 120002, 2018.

[21] S. Katz, E. Zaretsky, E. Grossman, and H. D. Wagner, "Dynamic tensile strength of organic fiber-reinforced epoxy micro-composites," Compos. Sci. Technol., vol. 69, no. 7-8, pp. 1250-1255, 2009.

[22] D. Grady, "The spall strength of condensed matter," J. Mech. Phys. Solids, vol. 36, pp. 353-384, jan 1988.

[23] V. Tvergaard and A. Needleman, "Analysis of the cup-cone fracture in a round tensile bar," Acta Metall., 1984. 
[24] D. A. Shockey, L. Seaman, and D. R. Curran, "The Influence of Microstructural Features on Dynamic Fracture," in Metall. Eff. High Strain Rates, pp. 473-499, Boston, MA: Springer US, 1973.

[25] W. Wang, H. Zhang, M. Yang, P. Jiang, F. Yuan, and X. Wu, "Shock and spall behaviors of a high specific strength steel: Effects of impact stress and microstructure," $J$. Appl. Phys., vol. 121, no. 13, 2017.

[26] R. S. Hixson, J. N. Johnson, G. T. Gray, and J. D. Price, "Effects of interfacial bonding on spallation in metal-matrix composites," in AIP Conf. Proc., vol. 370, pp. 555-558, AIP, 1996.

[27] G. T. Gray, N. K. Bourne, and B. L. Henrie, "On the influence of loading profile upon the tensile failure of stainless steel," J. Appl. Phys., vol. 101, no. 9, 2007.

[28] B. M. Butcher, L. M. Barker, D. E. Munson, and C. D. Lundergan, "Influence of stress history on time-dependent spall in metals," AIAA J., vol. 2, no. 6, pp. 977-990, 1965.

[29] J. N. Johnson, "Spallation studies in Estane," in AIP Conf. Proc., vol. 505, pp. 543546, AIP, 2000.

[30] D. R. Curran, D. A. Shockey, and L. Seaman, "Dynamic fracture criteria for a polycarbonate," J. Appl. Phys., vol. 44, no. 9, pp. 4025-4038, 1973.

[31] J. Huneault and A. J. Higgins, "Shock wave induced cavitation of silicone oils," J. Appl. Phys., vol. 125, no. 24, pp. 1-11, 2019.

[32] J. E. Pepper, J. Huneault, M. Rahmat, B. Ashrafi, and O. E. Petel, "The effect of curing agent on the dynamic tensile failure of an epoxy subjected to plate impact," Int. J. Impact Eng., vol. 113, pp. 203-211, mar 2018.

[33] B. X. Bie, J. H. Han, L. Lu, X. M. Zhou, M. L. Qi, Z. Zhang, and S. N. Luo, "Dynamic fracture of carbon nanotube/epoxy composites under high strain-rate loading," Compos. Part A Appl. Sci. Manuf., vol. 68, pp. 282-288, 2015.

[34] L. C. Tang, H. Zhang, J. hua Han, X. ping Wu, and Z. Zhang, "Fracture mechanisms of epoxy filled with ozone functionalized multi-wall carbon nanotubes," Compos. Sci. Technol., vol. 72, pp. 7-13, dec 2011.

[35] L. C. Tang, H. Zhang, X. P. Wu, and Z. Zhang, "A novel failure analysis of multiwalled carbon nanotubes in epoxy matrix," Polymer (Guildf)., vol. 52, pp. 2070-2074, apr 2011. 
[36] Z. A. M. Ishak, A. Aminullah, H. Ismail, and H. D. Rozman, "Effect of Silane-Based Coupling Agents and Acrylic Acid Based Compatibilizers on Mechanical Properties of Oil Palm Empty Fruit Bunch Filled High-Density Polyethylene Composites," $J$. Appl. Polym. Sci., vol. 68, pp. 2189-2203, 1997.

[37] Y. J. Wan, L. X. Gong, L. C. Tang, L. B. Wu, and J. X. Jiang, "Mechanical properties of epoxy composites filled with silane-functionalized graphene oxide," Compos. Part A Appl. Sci. Manuf., vol. 64, pp. 79-89, 2014.

[38] Y. Zhang and S. J. Park, "Effect of Mercapto-Terminated Silane Treatment on Rheological and Mechanical Properties of Rice Bran Carbon-Reinforced Nitrile Butadiene Rubber Composites,” Macromol. Res., vol. 26, no. 5, pp. 446-453, 2018.

[39] M. N. Ansari and H. Ismail, "The effect of silane coupling agent on mechanical properties of feldspar filled polypropylene composites," J. Reinf. Plast. Compos., vol. 28, no. 24, pp. 3049-3060, 2009.

[40] C. B. N. McCrum, C. Buckley, Principles of Polymer Engineering. New York, NY: Oxford University Press Inc, 2 ed., 1997.

[41] C. Comtois-Arnaldo, The Ballistic Response of Particle-filled Elastomeric Systems. Master's thesis, Carleton University, 2018.

[42] V. Kushvaha and H. Tippur, "Dynamic Behavior of Materials, Volume 1," in Conf. Proc. Soc. Exp. Mech. Ser. (V. Chalivendra, B. Song, and D. Casem, eds.), vol. 4 of Conference Proceedings of the Society for Experimental Mechanics Series, ch. 66, pp. 513-522, New York, NY: Springer New York, 2013.

[43] G. I. Kanel, A. V. Utkin, and Z. G. Tolstikova, "Response of the high-filled elastomers to shock-wave loading," in AIP Conf. Proc., vol. 309, pp. 1123-1125, AIP, 1994.

[44] D. Grady, "Physics of Shock and Impact, Volume 1," in Phys. Shock Impact, Vol. 1, ch. 1, pp. 1.1-1.103, Philadelphia, PA: IOP Publishing, 1 ed., dec 2017.

[45] P. J. Hazell, Armour. Boca Raton, FL: CRC Press, 1st ed., jul 2015.

[46] M. A. Meyers, Dynamic Behavior of Materials. Wiley, sep 1994.

[47] D. R. Curran, L. Seaman, and D. A. Shockey, "Dynamic failure of solids," 1987.

[48] C. Williams and B. Love, "Dynamic Failure of Materials: A Review," tech. rep., U.S. Army Research Laboratory, Aberdeen Proving Ground, MD 21005-5066, 2010. 
[49] N. K. Bourne, Z. Rosenberg, D. J. Johnson, J. E. Field, A. E. Timbs, and R. P. Flaxman, "Design and construction of the UK plate impact facility," Meas. Sci. Technol., vol. 6, pp. 1462-1470, oct 1995 .

[50] G. I. Kanel, S. V. Razorenov, K. Baumung, and J. Singer, "Dynamic yield and tensile strength of aluminum single crystals at temperatures up to the melting point," J. Appl. Phys. J. Appl. Phys. J. Appl. Phys. J. Appl. Phys. J. Appl. Phys. J. Appl. Phys. J. Appl. Phys., vol. 90, no. 58, pp. 2330-23528, 2001.

[51] N. A. Pedrazas, D. L. Worthington, D. A. Dalton, P. A. Sherek, S. P. Steuck, H. J. Quevedo, A. C. Bernstein, E. M. Taleff, and T. Ditmire, "Effects of microstructure and composition on spall fracture in aluminum," Mater. Sci. Eng. A, vol. 536, pp. 117$123,2012$.

[52] S. A. Novikov and A. G. Ivanov, "Failure of steel, aluminium and copper under explosive shock loading," Fiz. Met. i Metalloved., vol. 21, no. 4, pp. 608-615, 1966.

[53] G. I. Kanel, "Spall fracture: Methodological aspects, mechanisms and governing factors," Int. J. Fract., vol. 163, no. 1-2, pp. 173-191, 2010.

[54] G. V. Stepanov, "Spall fracture of metals by elastic-plastic loading waves," Probl Prochn, vol. 8, no. 1976, pp. 66-70, 1976.

[55] M. G. Grewe, T. R. Gururaja, T. R. Shrout, and R. E. Newnham, "Acoustic Properties of Particle/Polymer Composites for Ultrasonic Transducer Backing Applications," IEEE Trans. Ultrason. Ferroelectr. Freq. Control, vol. 37, no. 6, pp. 506-514, 1990.

[56] S. Lees, R. Gilmore, and P. Kranz, "Acoustic Properties of Tungsten-Vinyl Composites,” IEEE Trans. Sonics Ultrason., vol. 20, pp. 1-1, jan 1973.

[57] S. Lees and C. L. Davidson, "Ultrasonic Measurement of Some Mineral Filled Plastics,” IEEE Trans. Sonics Ultrason., vol. SU-24, no. 3, pp. 222-225, 1977.

[58] G. Plume and C. E. Rousseau, "Investigation into the spall strength of cast iron," Int. J. Nonlinear Sci. Numer. Simul., vol. 13, no. 2, pp. 195-199, 2012.

[59] S. Biwa, S. Idekoba, and N. Ohno, "Wave attenuation in particulate polymer composites: Independent scattering/absorption analysis and comparison to measurements," Mech. Mater., vol. 34, no. 10, pp. 671-682, 2002. 
[60] S. M. Pilgrim, Application of Piezoelectric Polymer Composites to Passive and Active Vibration Absorption. PhD thesis, THE Pennsylvania State Uuniversity, 1987.

[61] Gelest, "SILANE, SILICONE AND METAL-ORGANIC MATERIALS INNOVATION."

[62] I. D. Johnston, D. K. McCluskey, C. K. Tan, and M. C. Tracey, "Mechanical characterization of bulk Sylgard 184 for microfluidics and microengineering," J. Micromechanics Microengineering, vol. 24, no. 3, 2014.

[63] J. C. F. Millett, G. Whiteman, S. M. Stirk, and N. K. Bourne, "Shear strength measurements in a shock loaded commercial silastomer,' J. Phys. D Appl. Phys, vol. 44, pp. 185403-185409, 2011.

[64] S. P. Marsh, "LASL Shock Hugoniot Data," Los Alamos Ser. Dyn. Mater. Prop., p. $150,1980$.

[65] A. E. Seigel, Theory of High Speed Guns. White Oak, Silver Spring, Maryland: NATO / United States Naval Ordinance Laboratory, 1965.

[66] O. T. Strand, D. R. Goosman, C. Martinez, T. L. Whitworth, and W. W. Kuhlow, "Compact system for high-speed velocimetry using heterodyne techniques," Rev. Sci. Instrum., vol. 77, p. 083108, aug 2006.

[67] P. Rae and E. Brown, "Some Observations on Measuring Sound Speeds in Polymers Using Time-of-Flight," Exp. Tech., pp. n/a-n/a, 2015.

[68] O. Technologies, "Olympus Inspection Solutions."

[69] O. E. Petel and A. J. Higgins, "Shock wave propagation in dense particle suspensions," J. Appl. Phys., 2010.

[70] P. Carpena, "Velocity measurements through magnetic induction," Am. J. Phys., vol. 65 , no. 2, pp. 135-140, 1997.

[71] O. T. Strand, D. R. Goosman, C. Martinez, T. L. Whitworth, and W. W. Kuhlow, "A Novel System for High- Speed Velocimetry Using Heterodyne Techniques," Rev. Sci. Instrum., 2005.

[72] J. G. Mance, B. M. La Lone, D. H. Dolan, S. L. Payne, D. L. Ramsey, and L. R. Veeser, "Time-stretched photonic Doppler velocimetry," Opt. Express, vol. 27, no. 18, p. 25022, 2019. 
[73] V. A. Kuznetsov and S. P. Bennetts, "Development of Photon Doppler Velocimeter for Explosives Research,” tech. rep., Australian Government Department of Defence DST, Edinburgh, South Australia, 2013.

[74] C. U. E. Department, ed., Materials Data Book, vol. 9. 2003 editi ed., 2003.

[75] R. G. Barsoum, ELASTOMERIC POLYMERS WITH HIGH RATE SENSITIVITY: Applications in Blast, Shockwave, and Penetration Mechanics. Elsevier, 1st ed.

[76] J. Pepper, An Experimental Investigation of Spall Fracture in Neat Epoxy and an Epoxy/Carbon Nanotube Composite. Master's thesis, Carleton University.

[77] C. L. Williams, C. Q. Chen, K. T. Ramesh, and D. P. Dandekar, "On the shock stress, substructure evolution, and spall response of commercially pure 1100-O aluminum," Mater. Sci. Eng. A, vol. 618, pp. 596-604, 2014.

[78] D. D. Koller, R. S. Hixson, G. T. Gray, P. A. Rigg, L. B. Addessio, E. K. Cerreta, J. D. Maestas, and C. A. Yablinsky, "Influence of shock-wave profile shape on dynamically induced damage in high-purity copper," J. Appl. Phys., vol. 98, no. 10, 2005.

[79] E. P. Plueddemann, "Silane Coupling Agents," Addit. Plast., pp. 123-167, 1978.

[80] B. Arkles, "Tailoring Surfaces with Silanes," Chemtech, vol. 7, no. 12, p. 766, 1977.

[81] X. Su and B. Shi, "Effect of silane coupling agents with different non-hydrolytic groups on tensile modulus of composite PDMS crosslinked membranes," React. Funct. Polym., vol. 98, pp. 1-8, 2016. 


\section{Appendix A}

\section{Tabulated Results}

This subsection serves as a reference and contains a complete tabulation of the results of the polyurethane spall testing, seen in Table A.1. For visualization, each experiment has been plotted by spall strength, strain rate, and sample type, as seen in Figure A.1.

\begin{tabular}{|c|c|c|c|c|c|c|c|c|}
\hline Sample & $\begin{array}{c}W_{\text {Sample }} \\
(\mathrm{mm})\end{array}$ & $\begin{array}{l}V_{\text {Flyer }} \\
(\mathrm{m} / \mathrm{s})\end{array}$ & Flyer Material & Striker & $\begin{array}{l}u_{\text {peak }} \\
(\mathrm{m} / \mathrm{s})\end{array}$ & $\begin{array}{l}u_{\text {pull }} \\
(\mathrm{m} / \mathrm{s})\end{array}$ & $\begin{array}{l}\sigma_{\text {spall }} \\
(M P a)\end{array}$ & $\begin{array}{c}\dot{\varepsilon} \\
\left(10^{4} s^{-1}\right)\end{array}$ \\
\hline Neat 1 & 8.4 & 431 & 1/8" PMMA & 1/16" PMMA & 459.0 & 317.5 & 167.4 & 3.0 \\
\hline Neat 2 & 8.4 & 291 & 1/8" PMMA & 1/16" РМMA & 296.4 & 144.0 & 180.4 & 2.5 \\
\hline Neat 3 & 8.1 & 286 & 1/8" Al. & 1/16" РMMA & 417.2 & 266.1 & 178.9 & 1.8 \\
\hline Neat 4 & 3.6 & 291 & 1/8" PMMA & N/A & 334.8 & 192.2 & 168.8 & 3.4 \\
\hline Neat 5 & 4.0 & 287 & 1/8" PMMA & N/A & 318.8 & 172.0 & 173.8 & 3.4 \\
\hline Spherical 1 & 11.1 & 437 & 1/8" PMMA & 1/16" PMMA & 417.2 & 316.5 & 174.9 & 1.9 \\
\hline Spherical 2 & 8.9 & 292 & 1/8" PMMA & 1/16" РMMA & 251.9 & 155.7 & 162.7 & 1.6 \\
\hline Spherical 3 & 8.7 & 576 & 1/8" РMMA & 1/16" РMМА & 524.6 & 403.5 & 210.4 & 2.0 \\
\hline Spherical 4 & 8.8 & 287 & 1/4" PMMA & 1/16" PMMA & 259.6 & 147.2 & 195.2 & 0.9 \\
\hline Irregular 1 & 8.7 & 439 & 1/8" PMMA & 1/16" PMMA & 367.5 & 275.8 & 160.0 & 1.6 \\
\hline Irregular 2 & 8.7 & 286 & 1/8" PMMA & 1/16" РММА & 210.5 & 95.6 & 200.5 & 1.5 \\
\hline Irregular 3 & 9.2 & 412 & 1/8" Al. & 1/16" PMMA & 431.8 & 339.1 & 161.9 & 1.1 \\
\hline Negative 1 & 4.5 & 427 & 1/8" PMMA & N/A & 417.5 & 381.7 & 56.9 & 2.2 \\
\hline Negative 2 & 4.8 & 402 & 1/8" PMMA & N/A & 382.4 & 358.3 & 38.4 & 1.6 \\
\hline Negative 3 & 4.7 & 396 & 1/8" Al. & N/A & 563.2 & 526.5 & 58.2 & 3.1 \\
\hline Negative 4 & 5.1 & 403 & 1/8" Al. & N/A & 603.5 & 566.0 & 59.6 & 3.8 \\
\hline Negative 5 & 10.2 & 548 & 1/8" PMMA & 1/16" PMMA & 479.5 & 428.9 & 81.5 & 1.9 \\
\hline PU+Silane 1 & 8.7 & 281 & 1/8" PMMA & 1/16" РMMA & 436.1 & 280 & 172.0 & 3.1 \\
\hline PU+Silane 2 & 8.2 & 290 & 1/8" Al. & 1/16" РMМА & 307.9 & 163.3 & 159.4 & 2.7 \\
\hline
\end{tabular}

Table A.1: Experimental results of the polyurethane spall strength study 


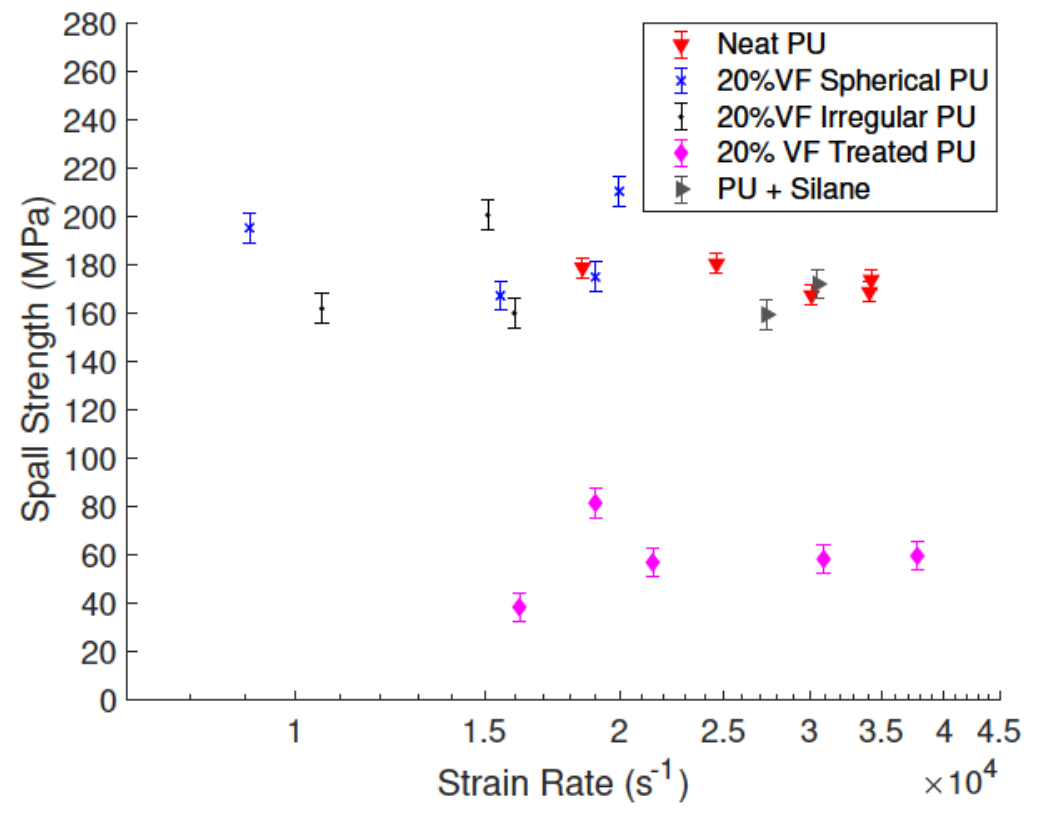

Figure A.1: Experimental spall strength measurements plotted against strain rate of the complete set of samples shot for this study 


\section{Appendix B}

\section{Spectrograms}

This appendix contains the velocity spectrograms of all of the experiments performed for the polyurethane surface adhesion study. As most experiments were monitored on two channels simultaneously each pair of spectrograms presented here denotes the free surface velocity of a single experiment. In some experiments only one PDV probe yielded usable results and as such is presented as a stand-alone for that experiment. The caption for each figure includes information regarding the striking velocity of the flyer plate, the material of the flyer plate as well as brief details regarding the sample itself.

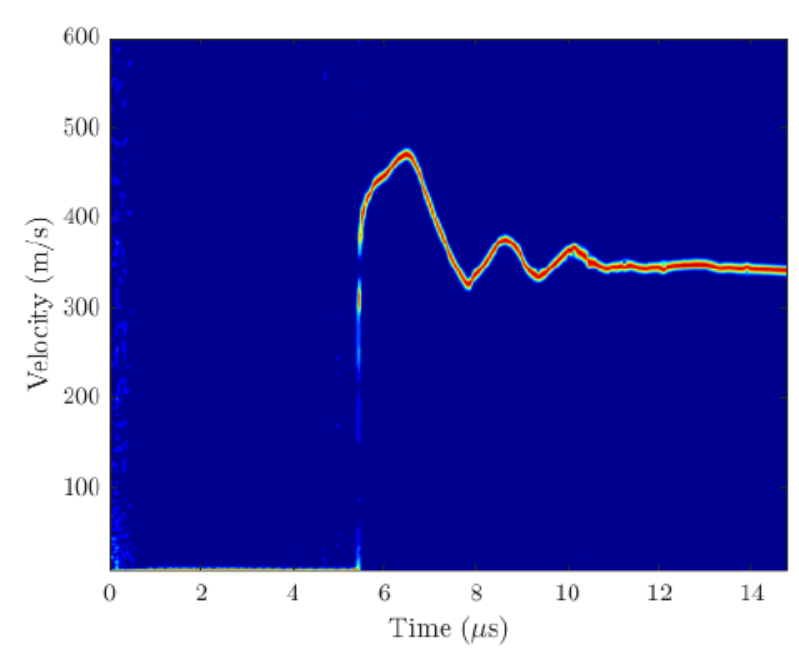

(a) Channel 3

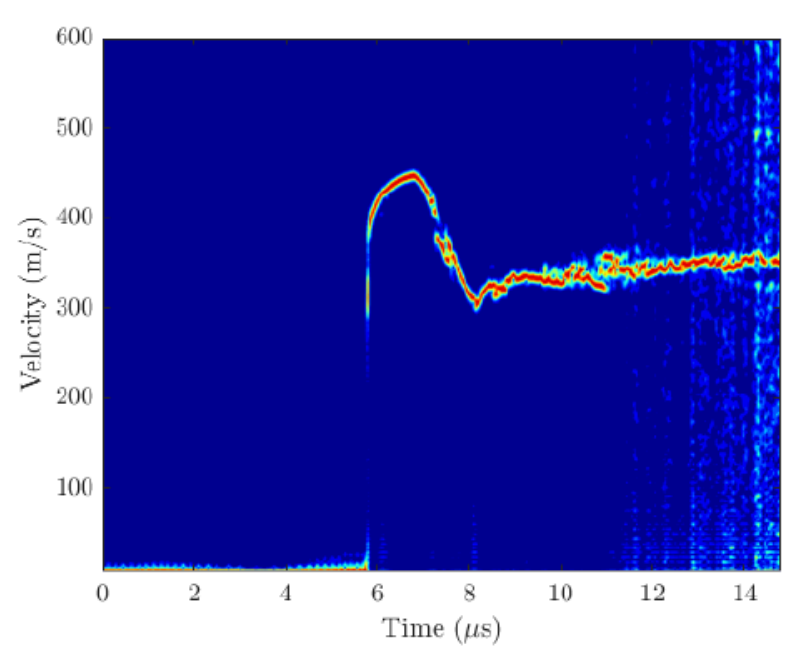

(b) Channel 4

Figure B.1: Spectrograms obtained from two PDV probes observing a Neat polyurethane (Neat 1) sample struck at $431 \mathrm{~m} / \mathrm{s}$ with an $1 / 8$ " acrylic striker 


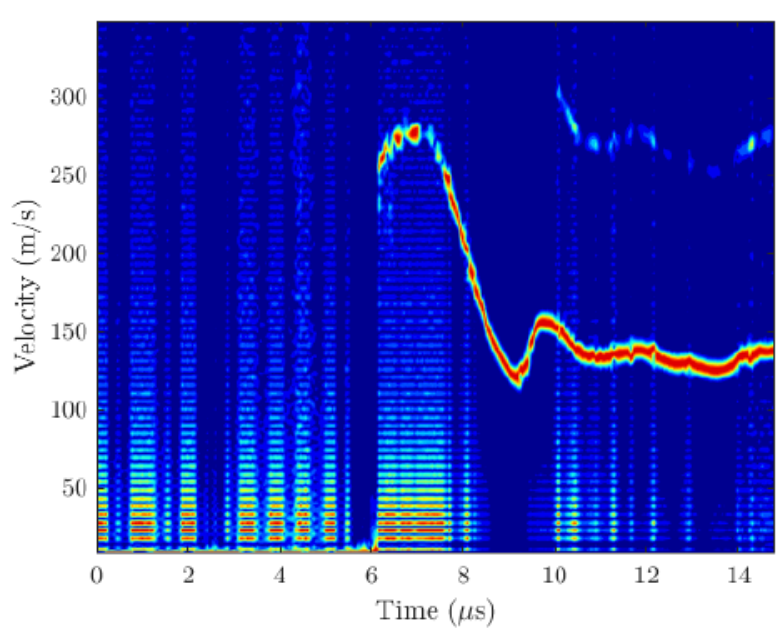

(a) Sample Struck at $290 \mathrm{~m} / \mathrm{s}$

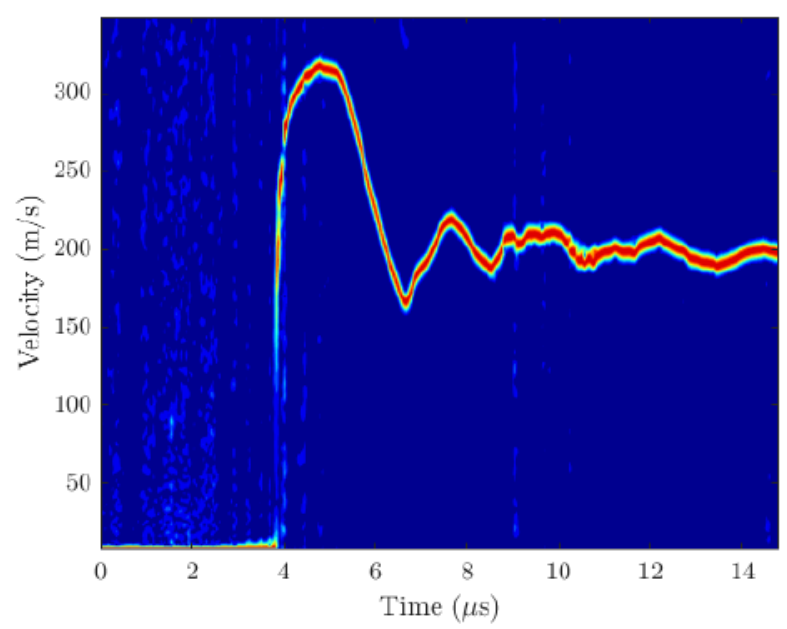

(b) Sample Struck at $292 \mathrm{~m} / \mathrm{s}$

Figure B.2: Spectrograms obtained from two separate (Neat 2) experiments observing a neat polyurethane sample struck with an $1 / 8$ " acrylic striker

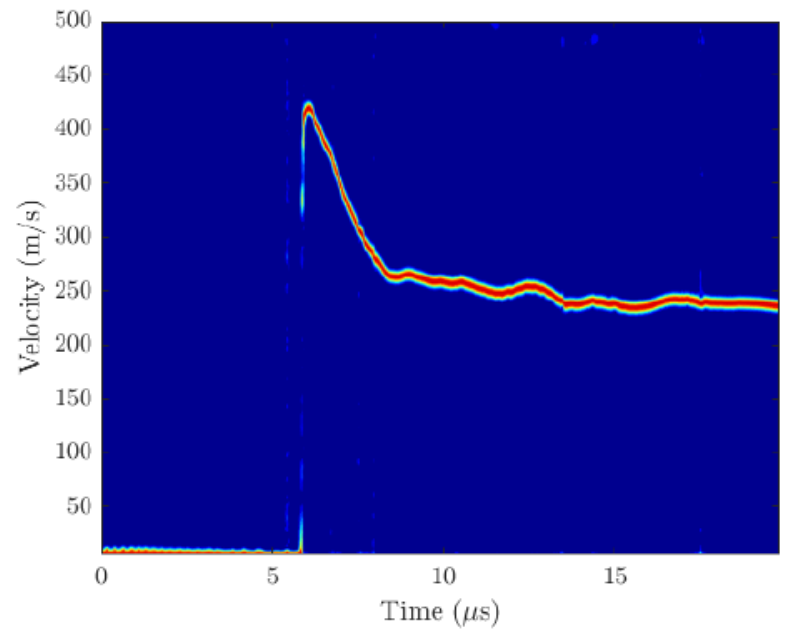

Figure B.3: Spectrograms obtained from two PDV probes observing a Neat polyurethane (Neat 3) sample struck at $286 \mathrm{~m} / \mathrm{s}$ with an $1 / 8$ " aluminium striker 


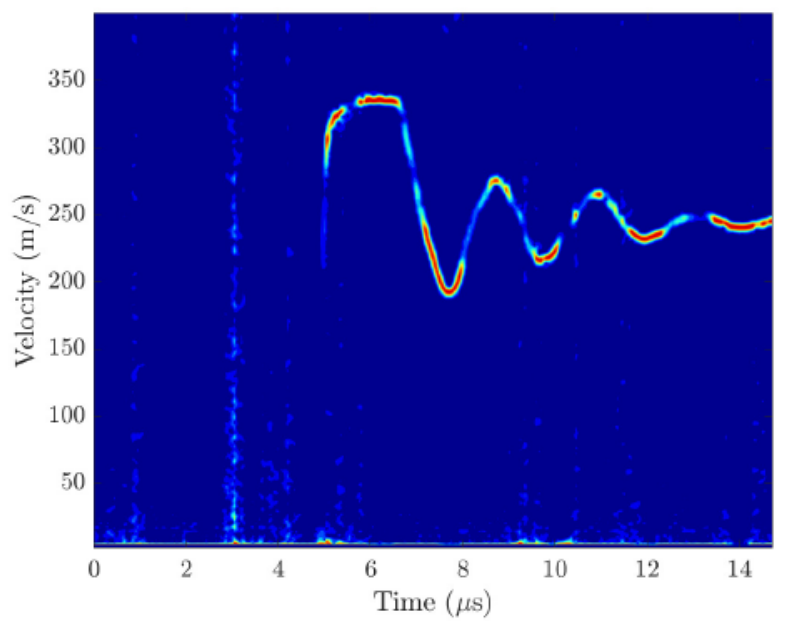

(a) Neat 4

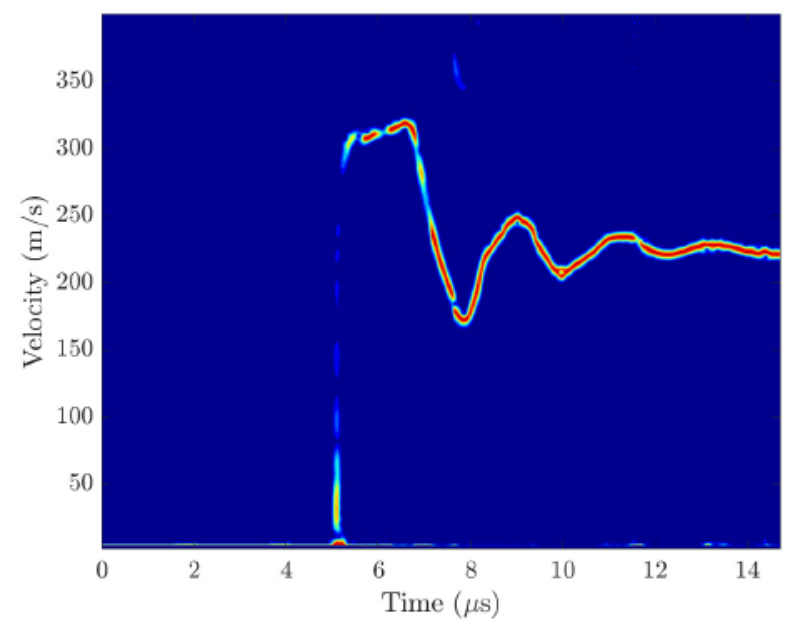

(b) Neat 5

Figure B.4: Spectrograms obtained from two separate experiments observing thin, neat polyurethane sample struck with an 1/8" acrylic striker

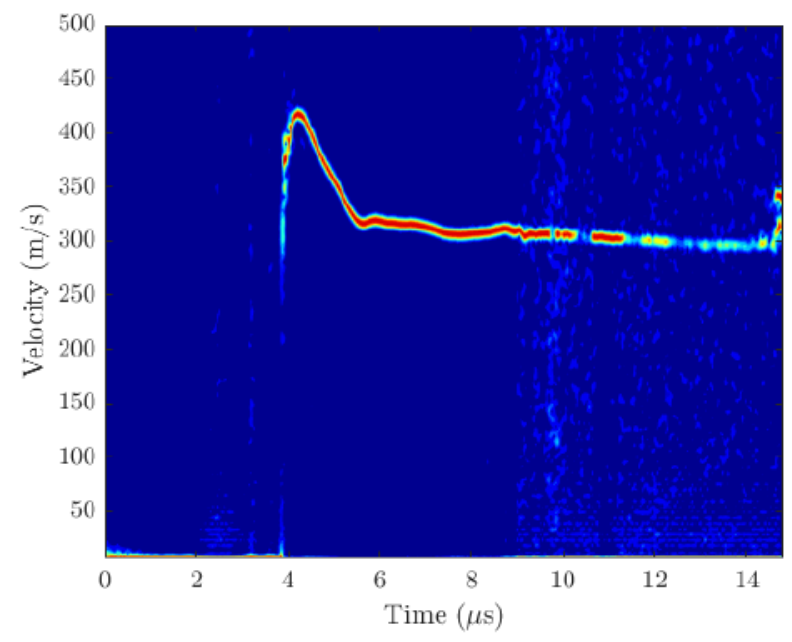

Figure B.5: Spectrograms obtained from two PDV probes observing a spherical alumina reinforced polyurethane (Spherical 1) sample struck at $436 \mathrm{~m} / \mathrm{s}$ with an 1/8" acrylic striker 


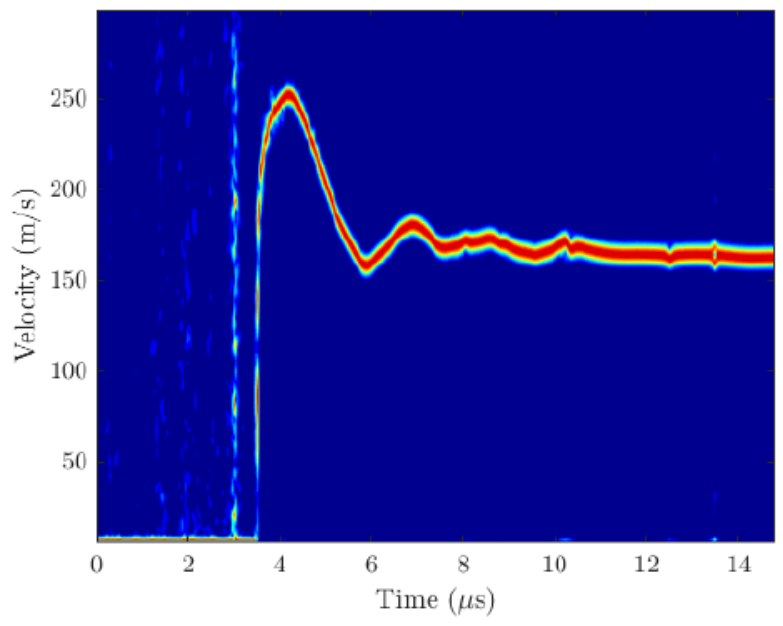

(a) Channel 3

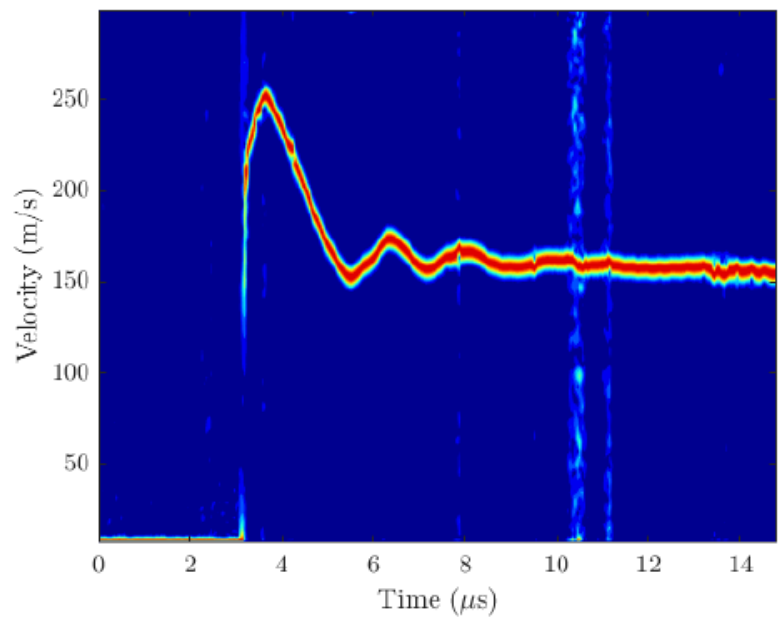

(b) Channel 4

Figure B.6: Spectrograms obtained from two PDV probes observing a Neat polyurethane (Spherical 2) sample struck at $292 \mathrm{~m} / \mathrm{s}$ with an 1/8" acrylic striker

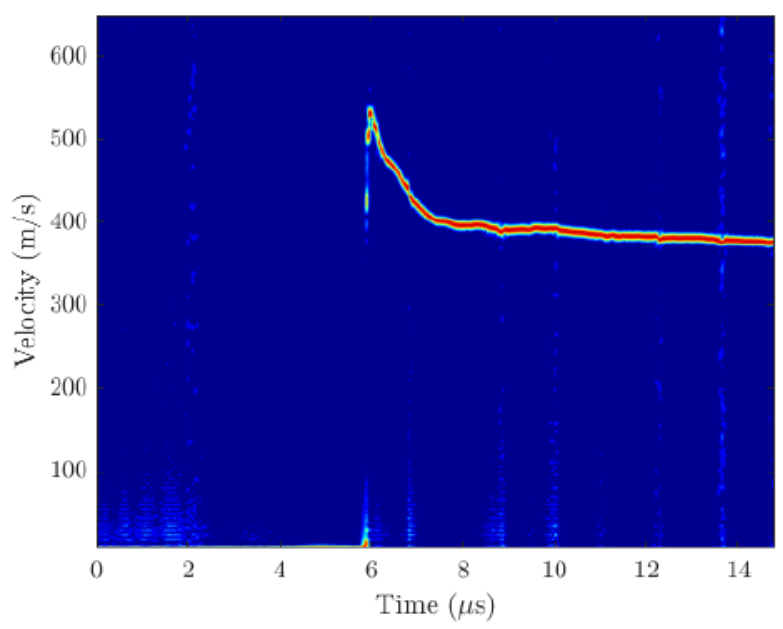

(a) Channel 3

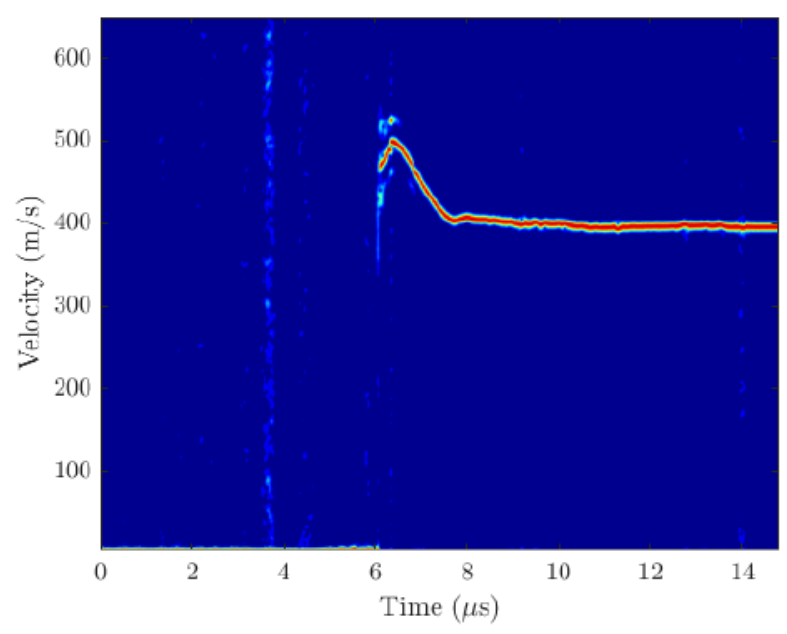

(b) Channel 4

Figure B.7: Spectrograms obtained from two PDV probes observing a spherical alumina reinforced polyurethane (Spherical 3) sample struck at $576 \mathrm{~m} / \mathrm{s}$ with an 1/8" acrylic striker. Peak was selected as max point in Channel 4, plots coincide at that time 


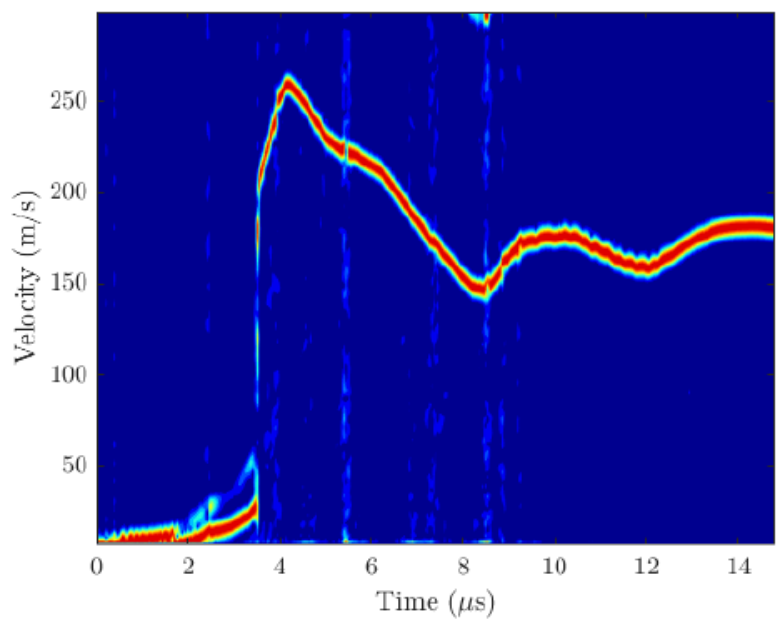

(a) Channel 3

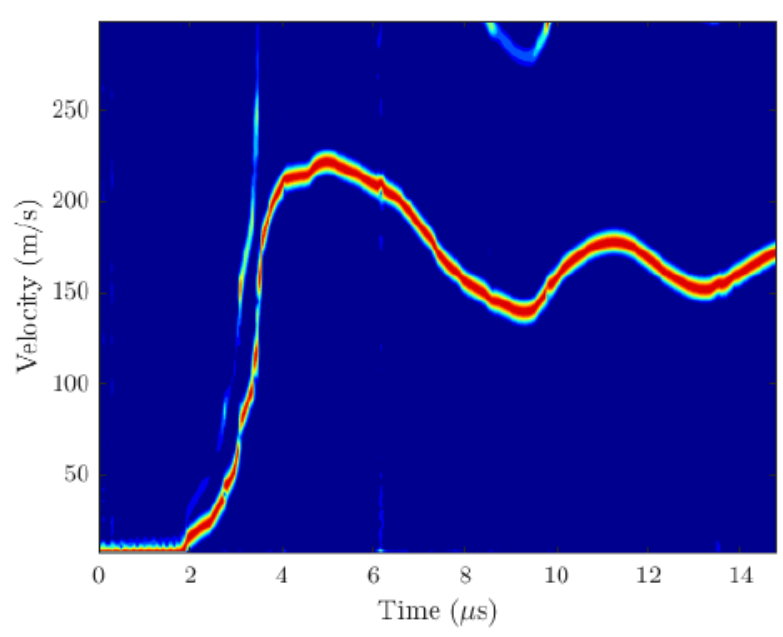

(b) Channel 4

Figure B.8: Spectrograms obtained from two PDV probes observing a spherical alumina reinforced polyurethane (Spherical 4) sample struck at $287 \mathrm{~m} / \mathrm{s}$ with an $1 / 4$ " aluminium striker. Peak was selected as max point in Channel 4, plots coincide at that time

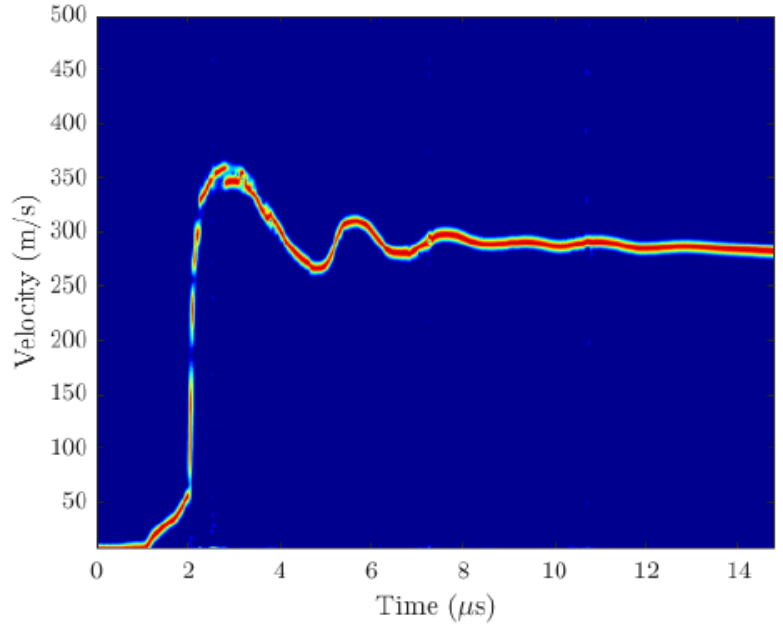

(a) Channel 3

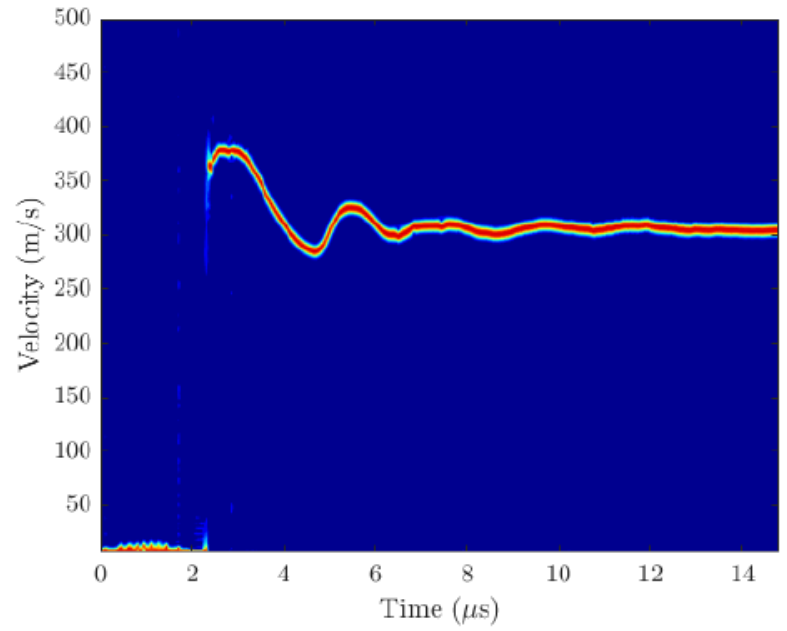

(b) Channel 4

Figure B.9: Spectrograms obtained from two PDV probes observing an irregular alumina reinforced polyurethane (Irregular 1) sample struck at $438 \mathrm{~m} / \mathrm{s}$ with an $1 / 8$ " acrylic striker 


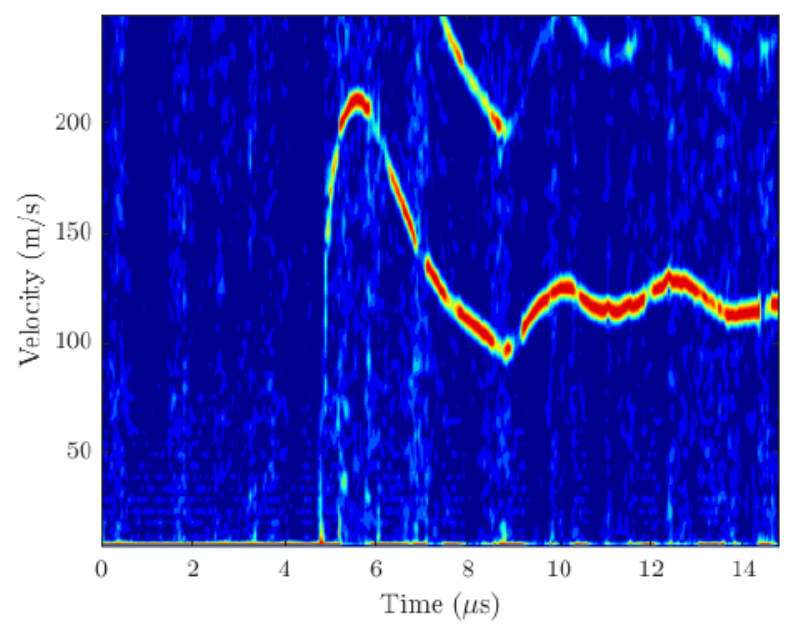

Figure B.10: Spectrograms obtained from a PDV probe observing an irregular alumina reinforced polyurethane (Irregular 2) sample struck at $286 \mathrm{~m} / \mathrm{s}$ with an 1/8" acrylic striker

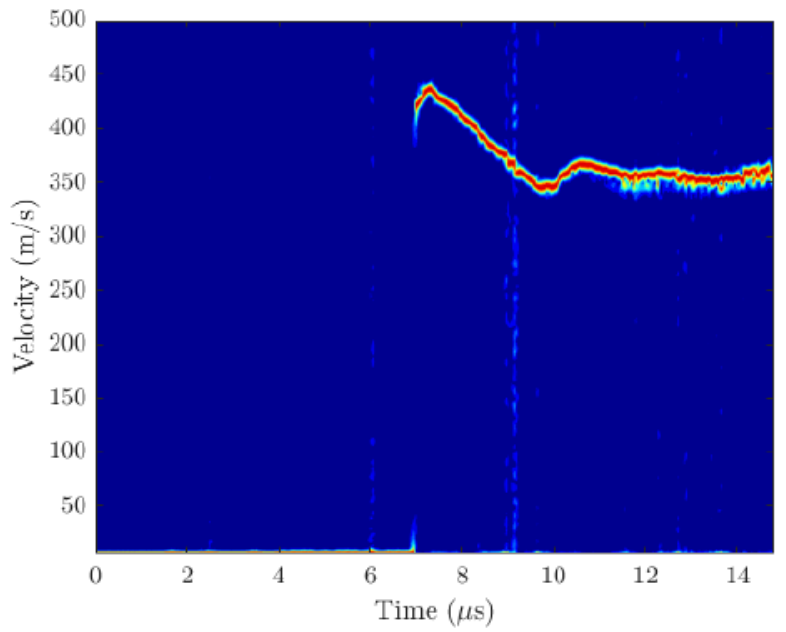

(a) Channel 3

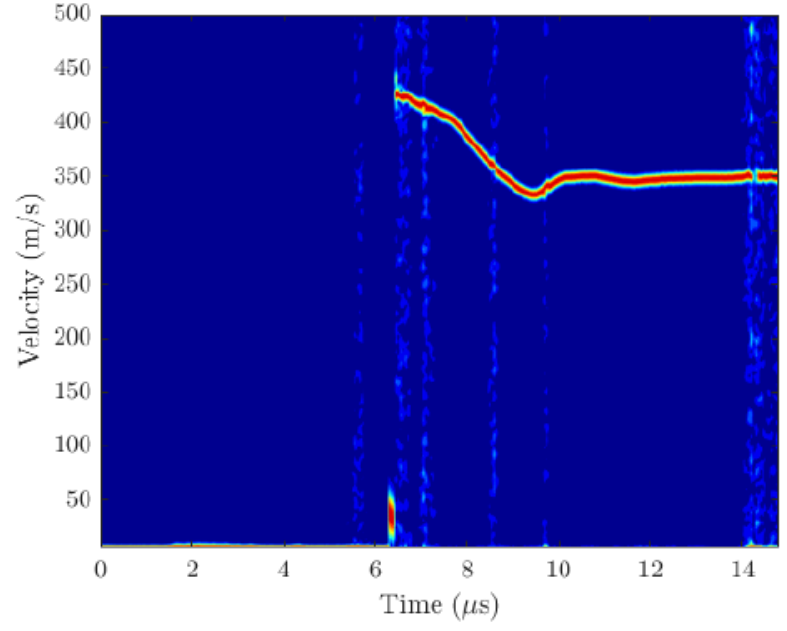

(b) Channel 4

Figure B.11: Spectrograms obtained from two PDV probes observing an irregular alumina reinforced polyurethane (Irregular 3) sample struck at $412 \mathrm{~m} / \mathrm{s}$ with an 1/8" aluminium striker 


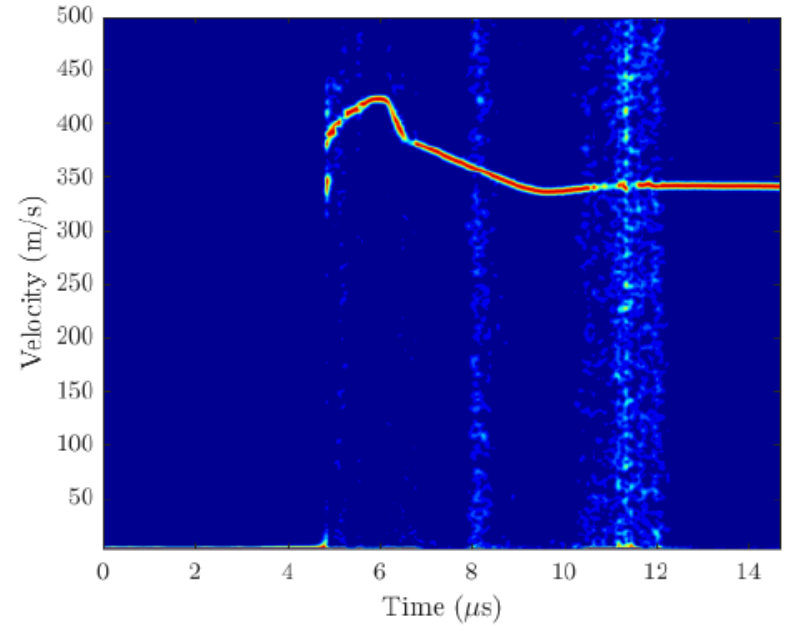

(a) Channel 3

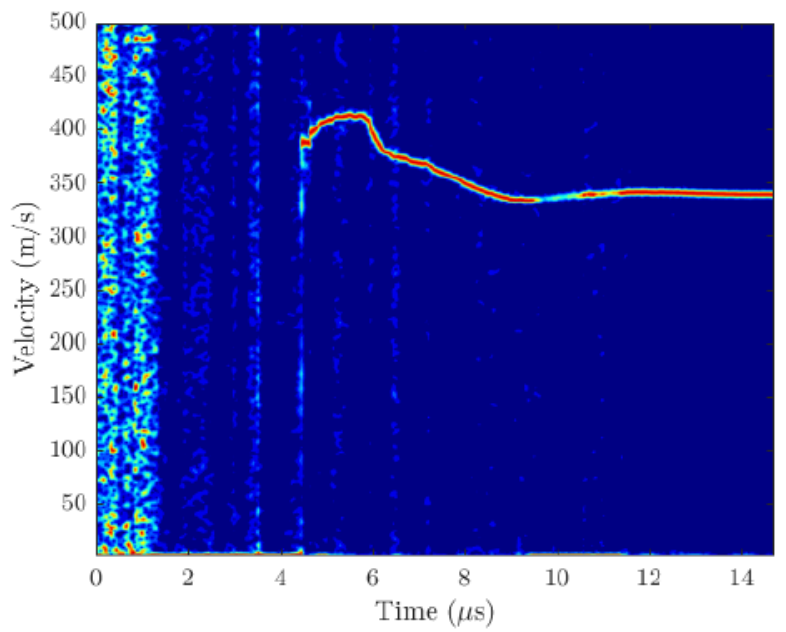

(b) Channel 4

Figure B.12: Spectrograms obtained from two PDV probes observing a silane treated spherical alumina reinforced polyurethane (Negative 1) sample struck at $427 \mathrm{~m} / \mathrm{s}$ with an $1 / 8$ " acrylic striker

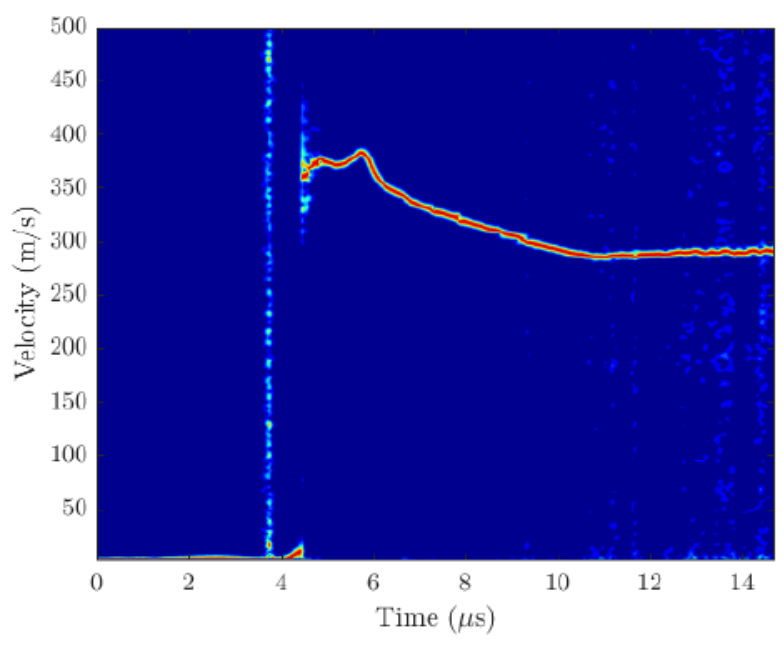

(a) Channel 3

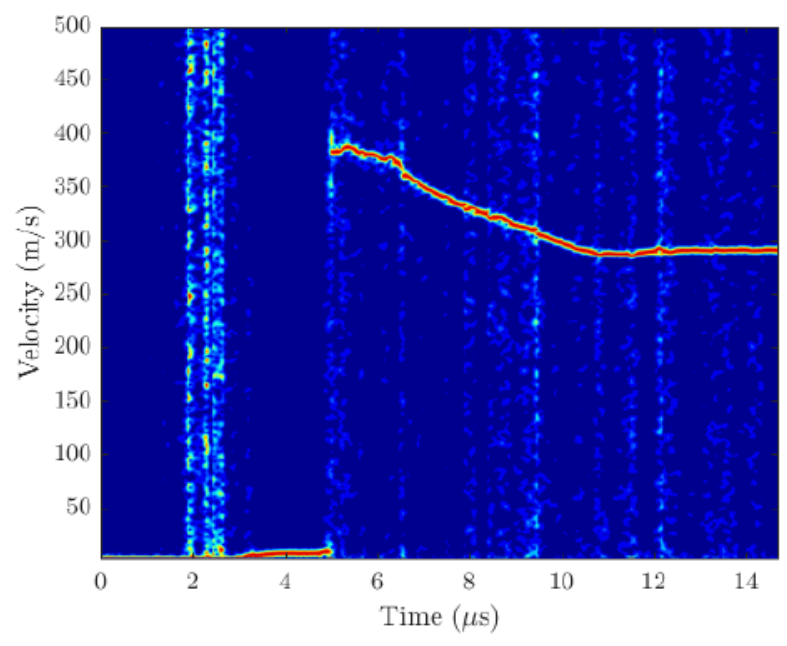

(b) Channel 4

Figure B.13: Spectrograms obtained from two PDV probes observing a silane treated spherical alumina reinforced polyurethane (Negative 2) sample struck at $402 \mathrm{~m} / \mathrm{s}$ with an $1 / 8$ " acrylic striker 


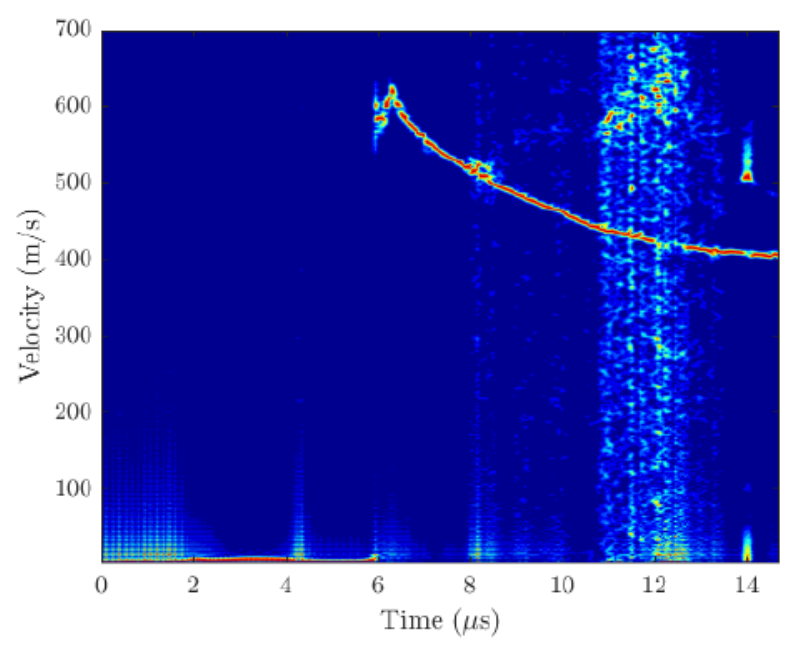

Figure B.14: Spectrogram obtained from a PDV probe observing a silane treated spherical alumina reinforced polyurethane (Negative 3) sample struck at $396 \mathrm{~m} / \mathrm{s}$ with an 1/8" aluminum striker

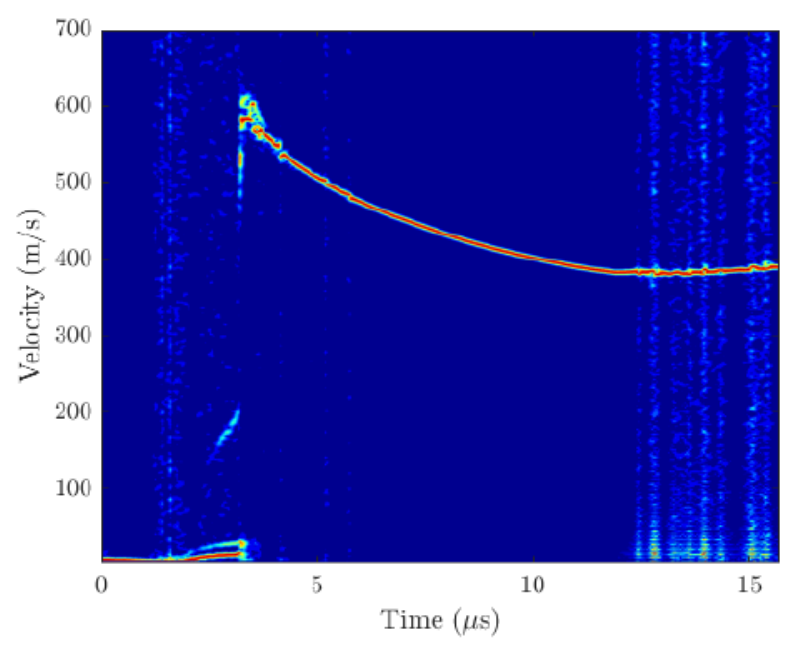

Figure B.15: Spectrograms obtained from a PDV probe observing a silane treated spherical alumina reinforced polyurethane (Negative 4) sample struck at $403 \mathrm{~m} / \mathrm{s}$ with an $1 / 8$ " aluminum 


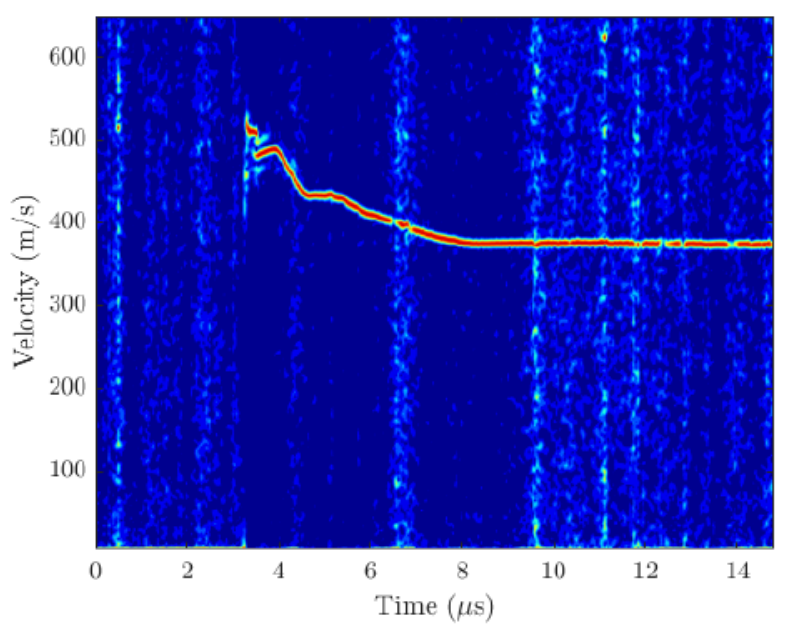

(a) Channel 3

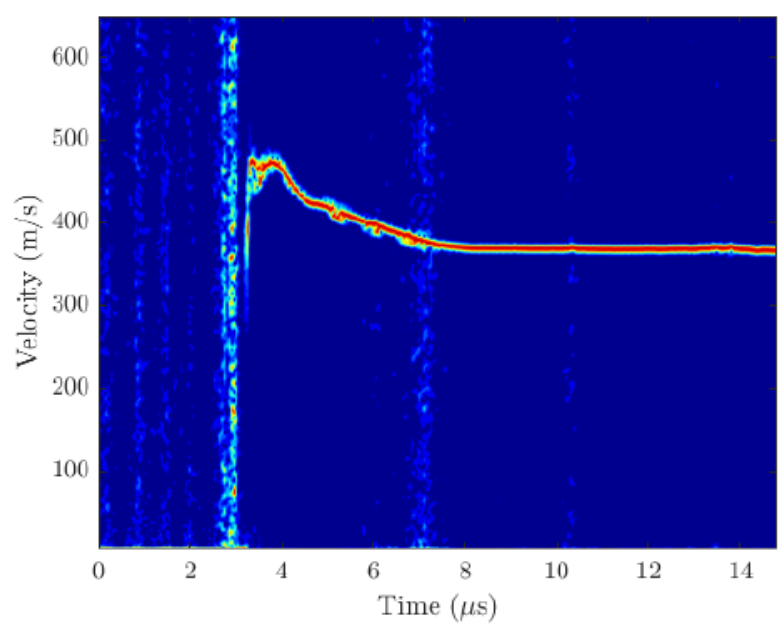

(b) Channel 4

Figure B.16: Spectrograms obtained from two PDV probes observing a silane treated spherical alumina reinforced polyurethane (Negative 5) sample struck at $548 \mathrm{~m} / \mathrm{s}$ with an $1 / 8$ " acrylic striker

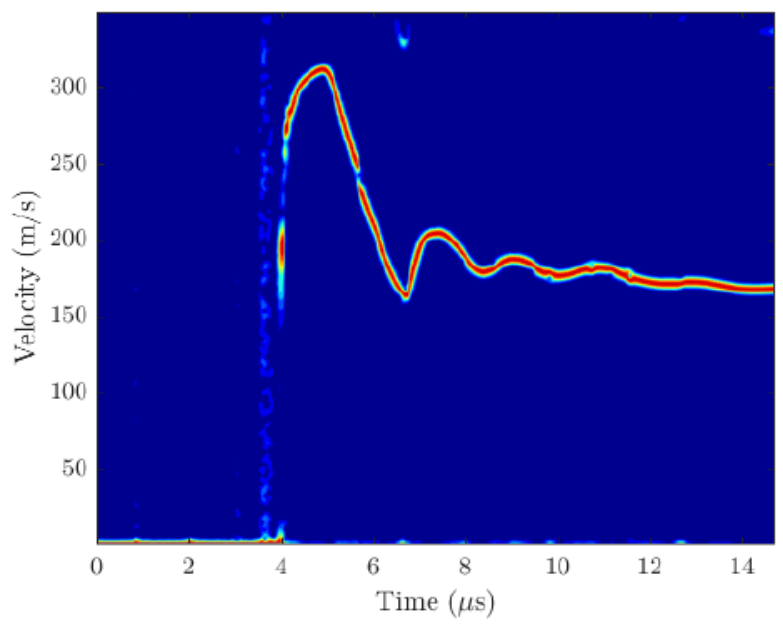

(a) Channel 3

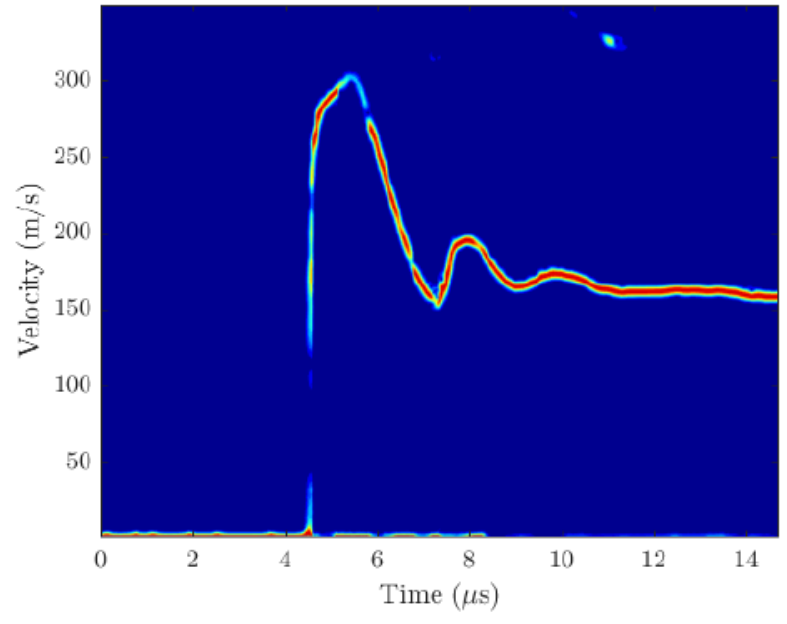

(b) Channel 4

Figure B.17: Spectrograms obtained from two PDV probes observing a silane treated spherical alumina reinforced polyurethane (PU+Silane 1) sample struck at $281 \mathrm{~m} / \mathrm{s}$ with an $1 / 8$ " acrylic striker 


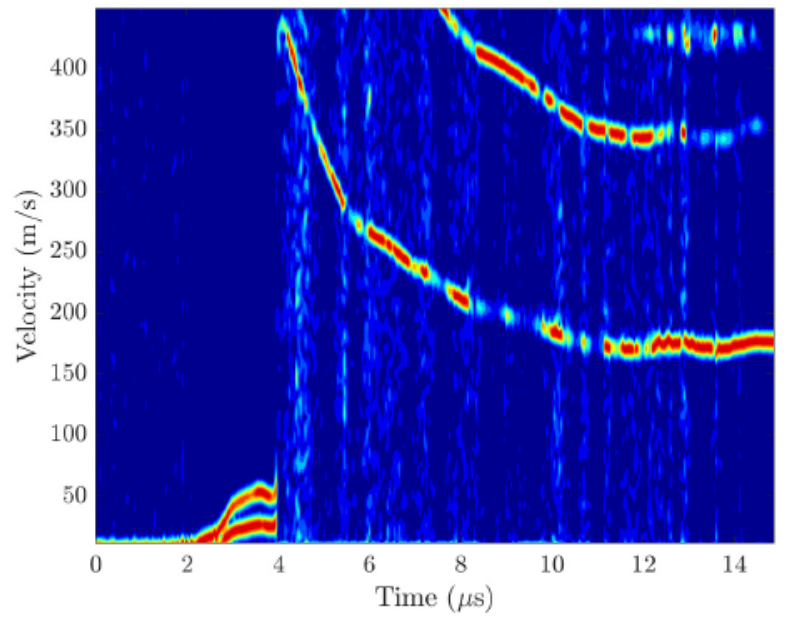

Figure B.18: Spectrograms obtained from a PDV probe observing a silane treated spherical alumina reinforced polyurethane (PU+Silane 2) sample struck at $290 \mathrm{~m} / \mathrm{s}$ with an $1 / 8$ " aluminum striker 


\section{Appendix C}

\section{SEM Imaging}

This appendix contains the collection of images obtained via Scanning Electron Microscopy Imaging. This appendix is sorted by sample type. Each image presented here shows a the fracture surface of the respective sample type with the line of site, normal to the fracture plane. If cyrogenic cutting was available samples could be prepared/sectioned to show an edge on view of the spall plane, while maintaining the spall induce damage. This was avoided in this study due to a lack of confidence in differentiating spall damage from damage caused by sectioning. 


\section{C.1 Imaging of the Neat Samples}
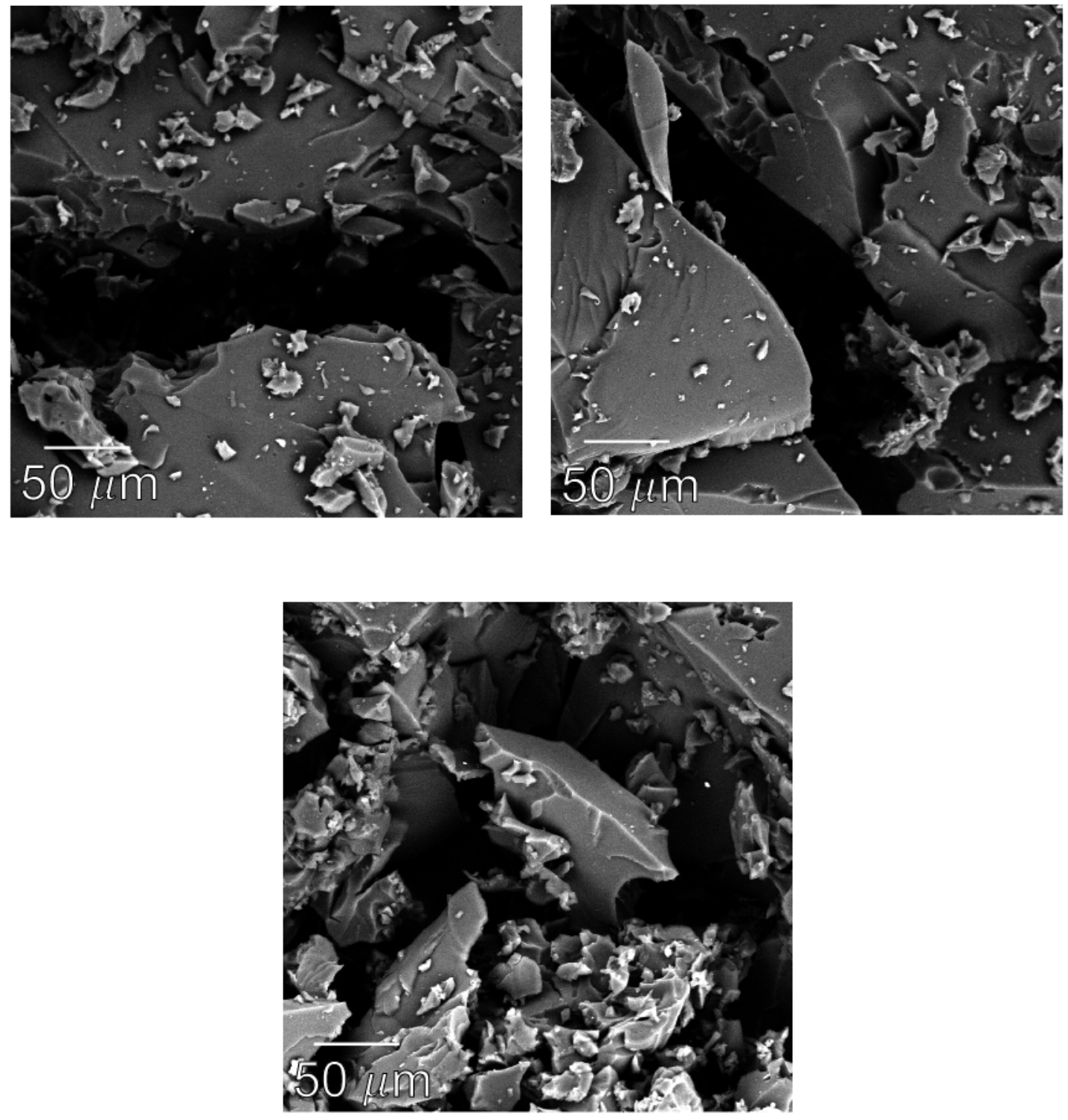


\section{C.2 Imaging of the Pristine Spherical Samples}
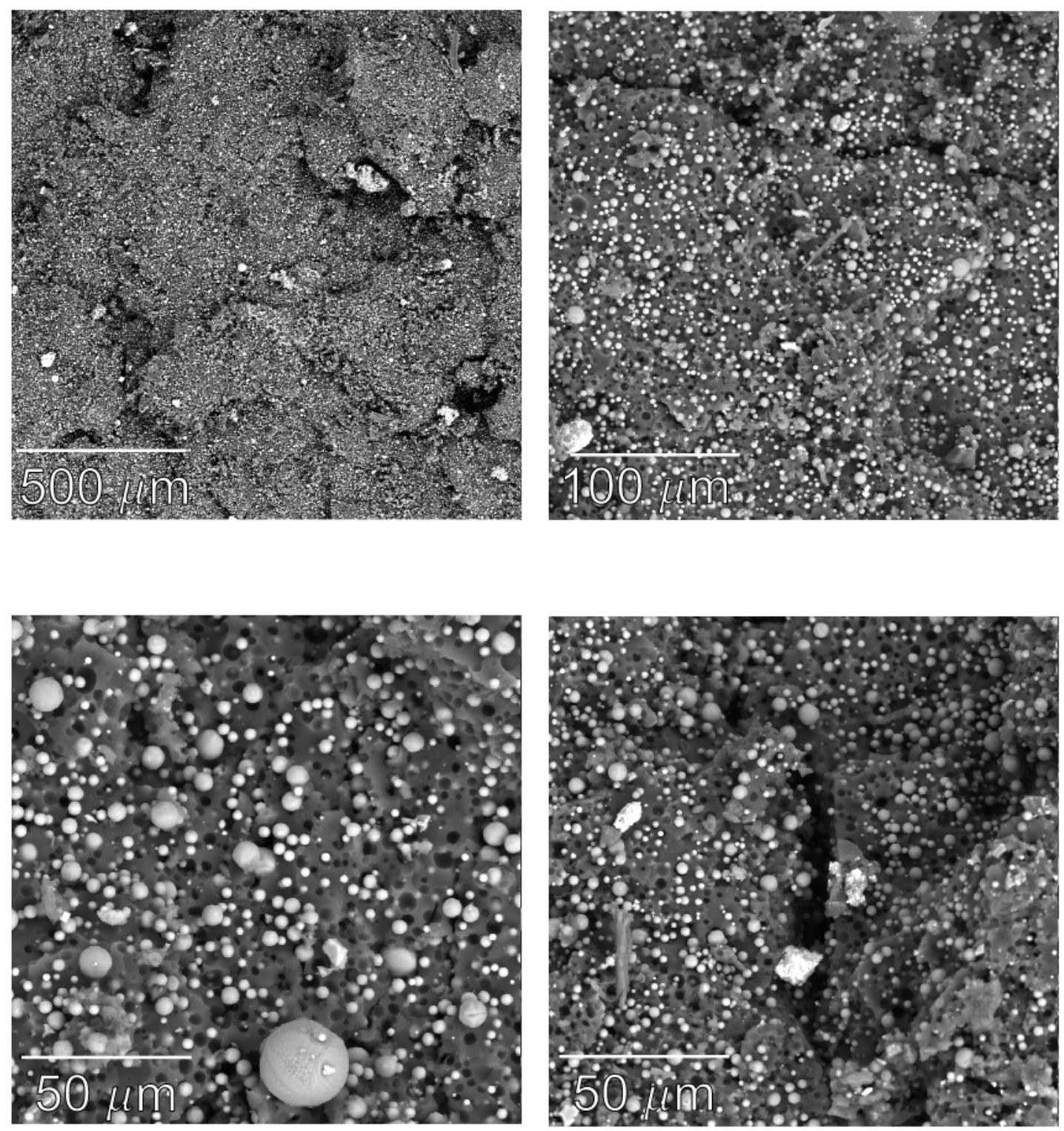

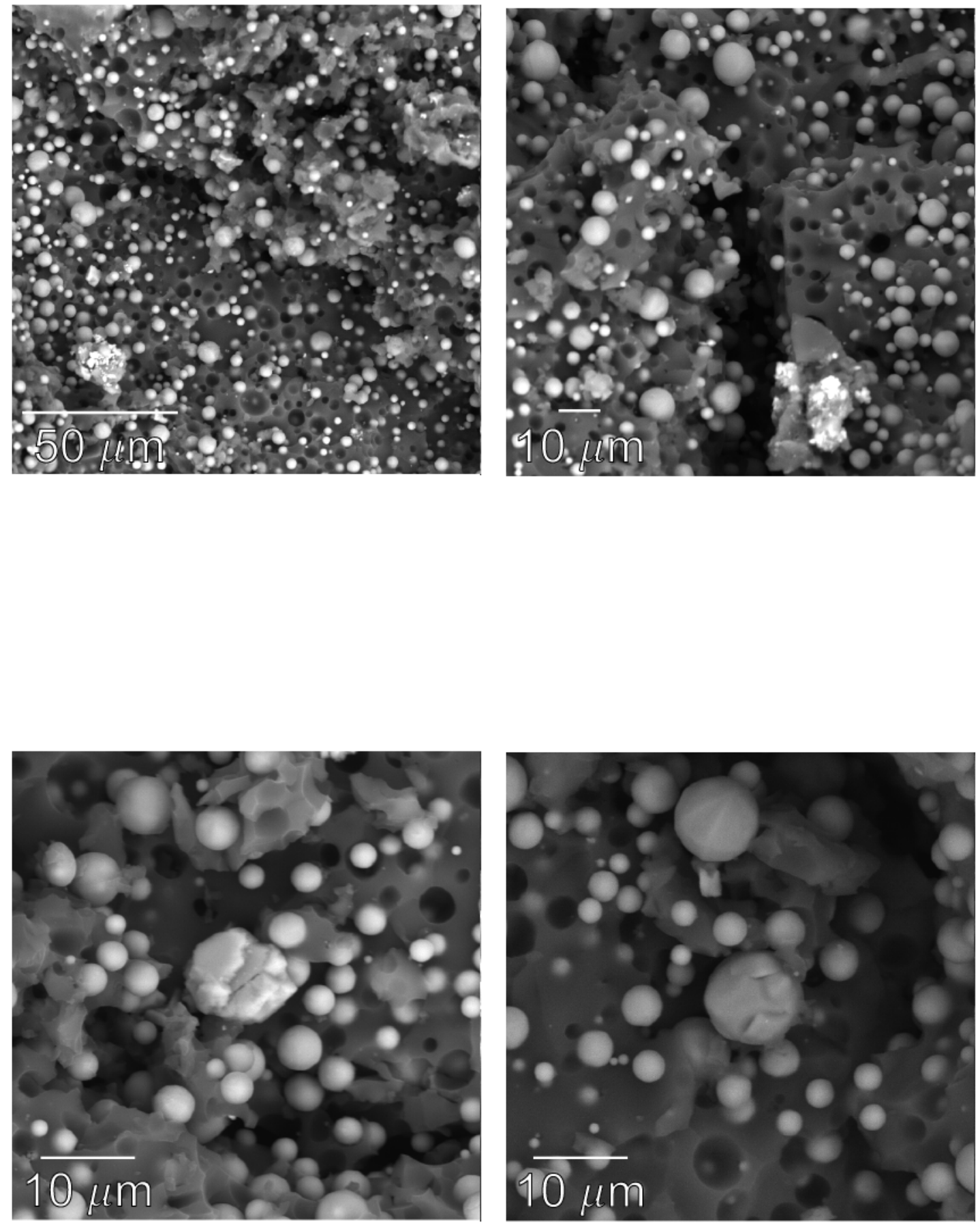


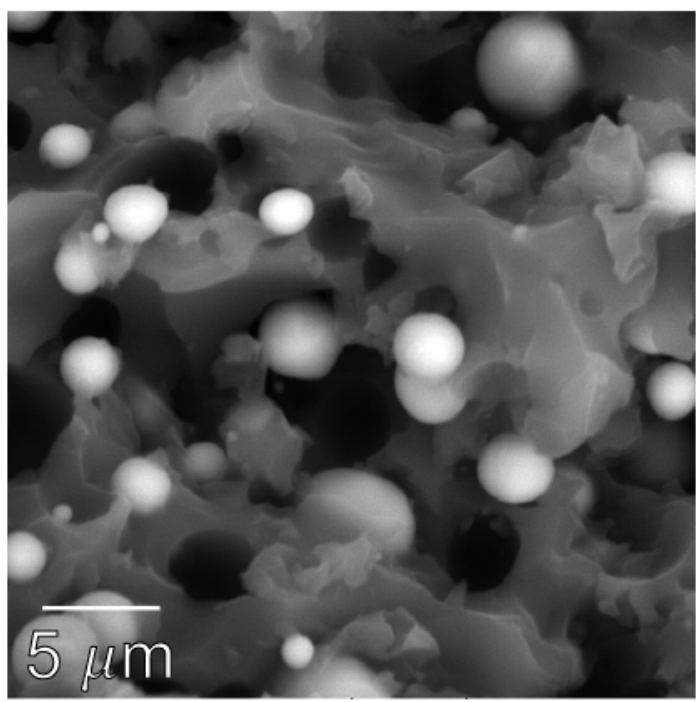

\section{C.3 Imaging of the Pristine Irregular Samples}
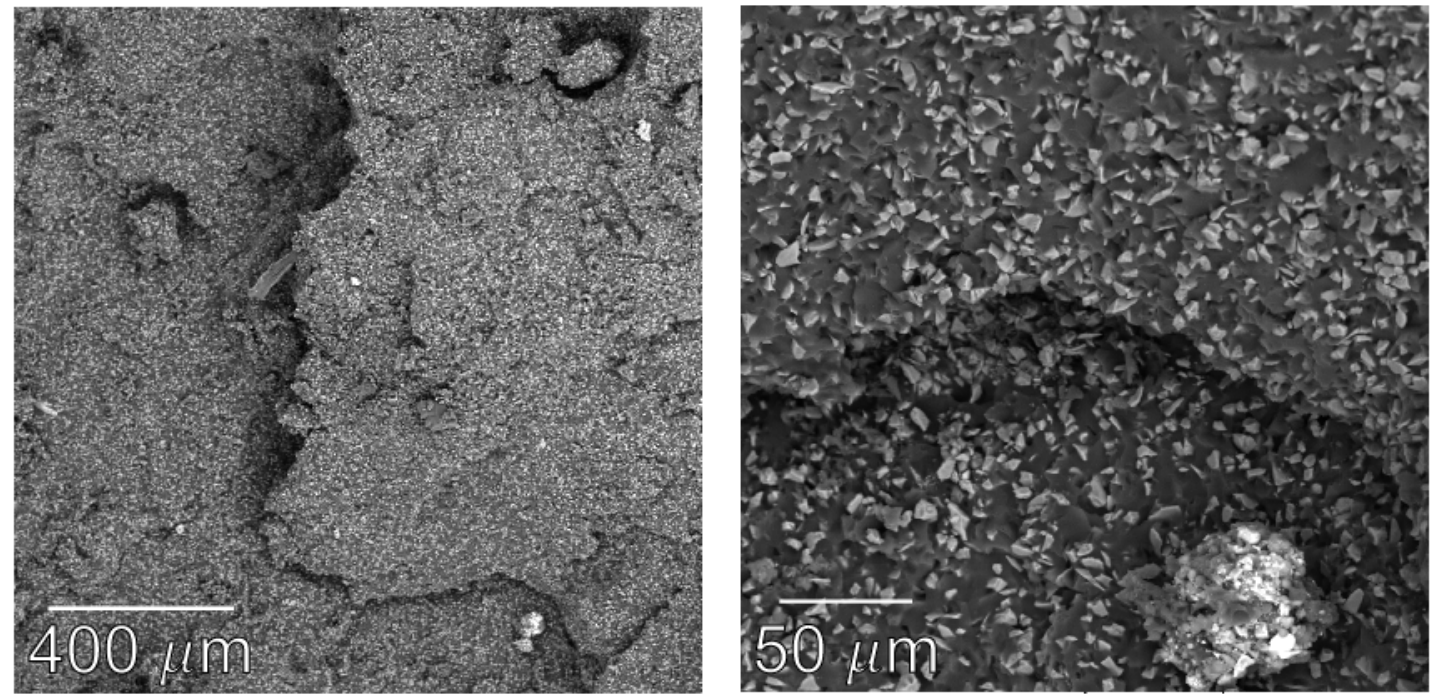

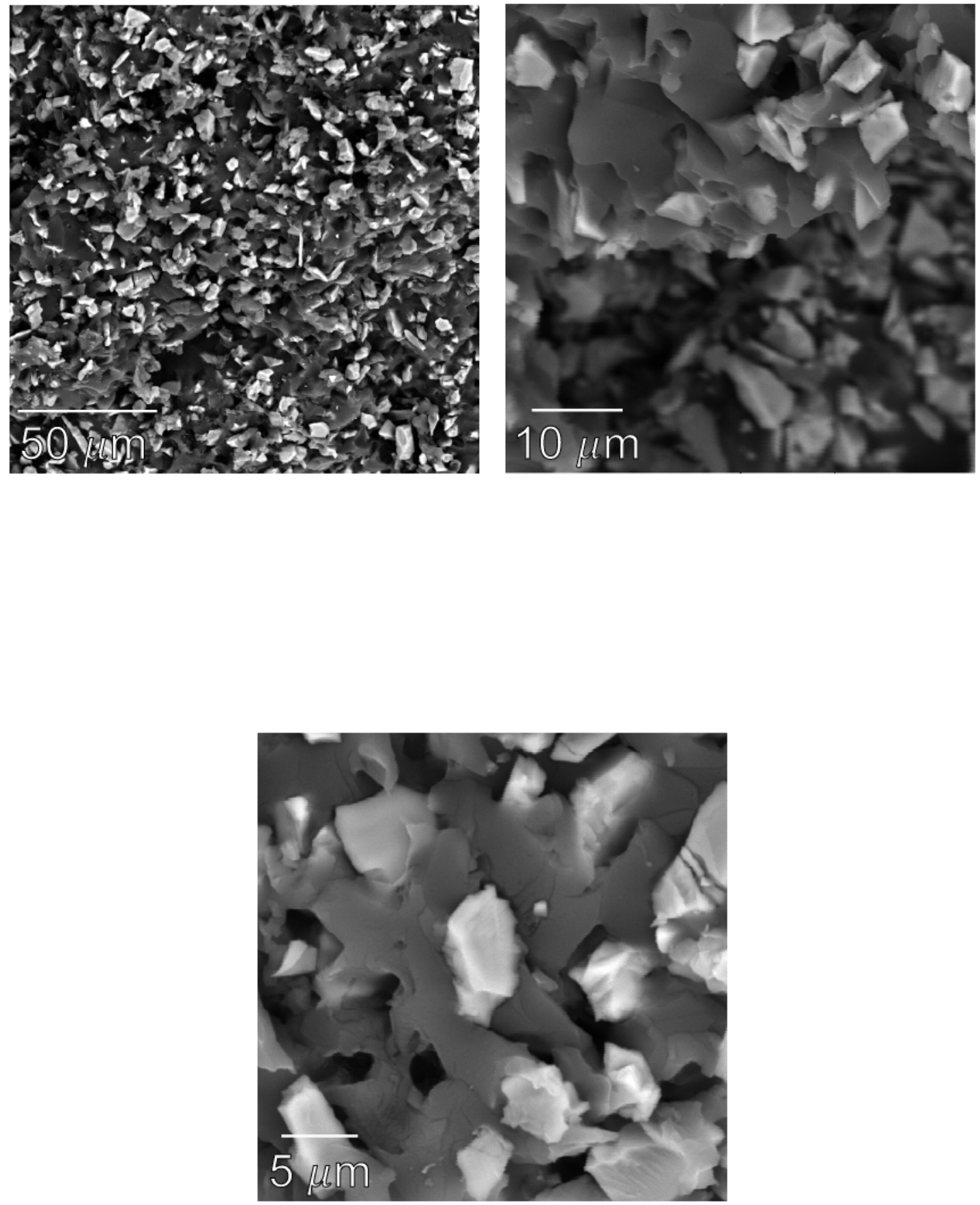


\section{C.4 Imaging of the Silane Treated Spherical Samples}
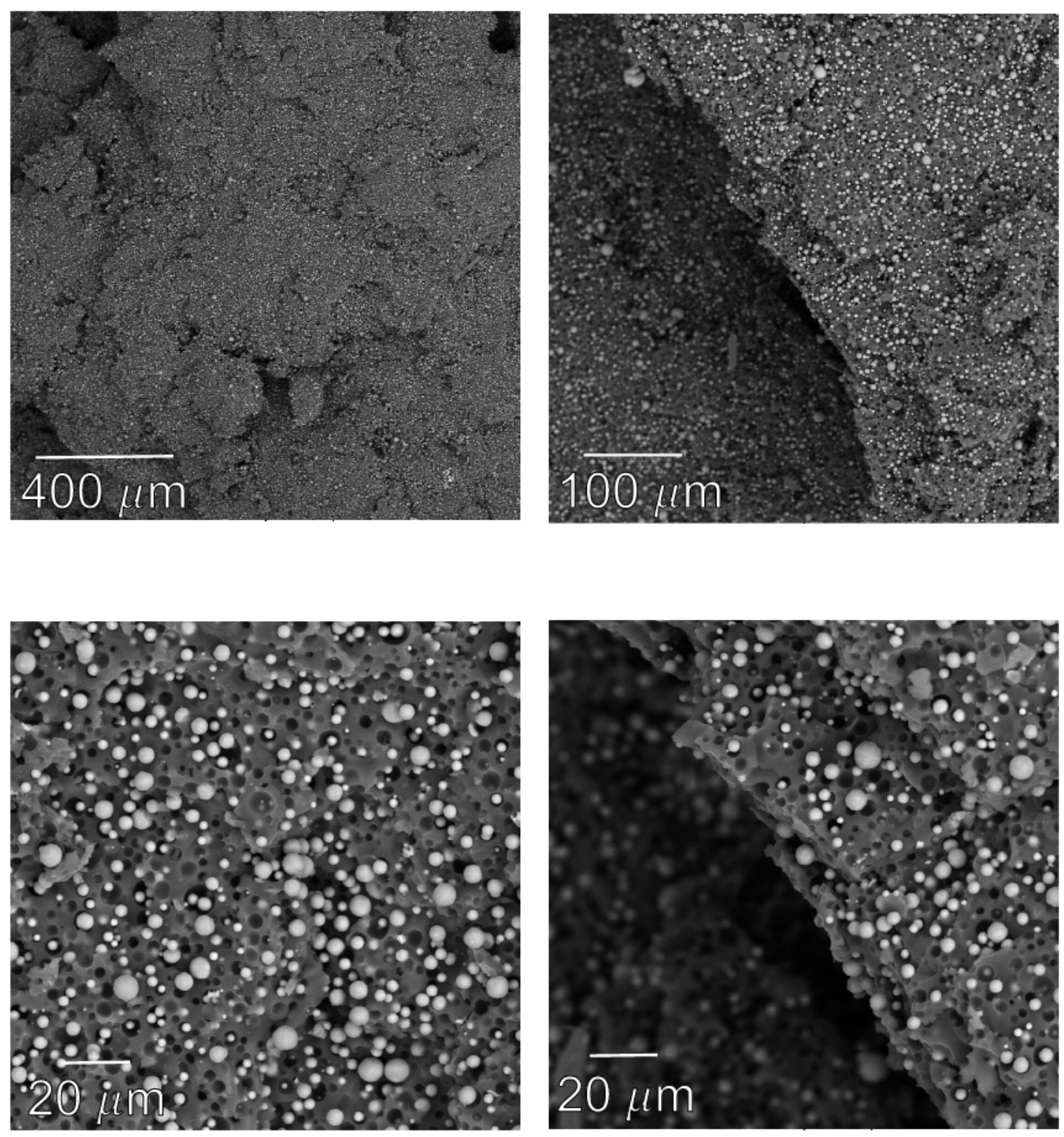

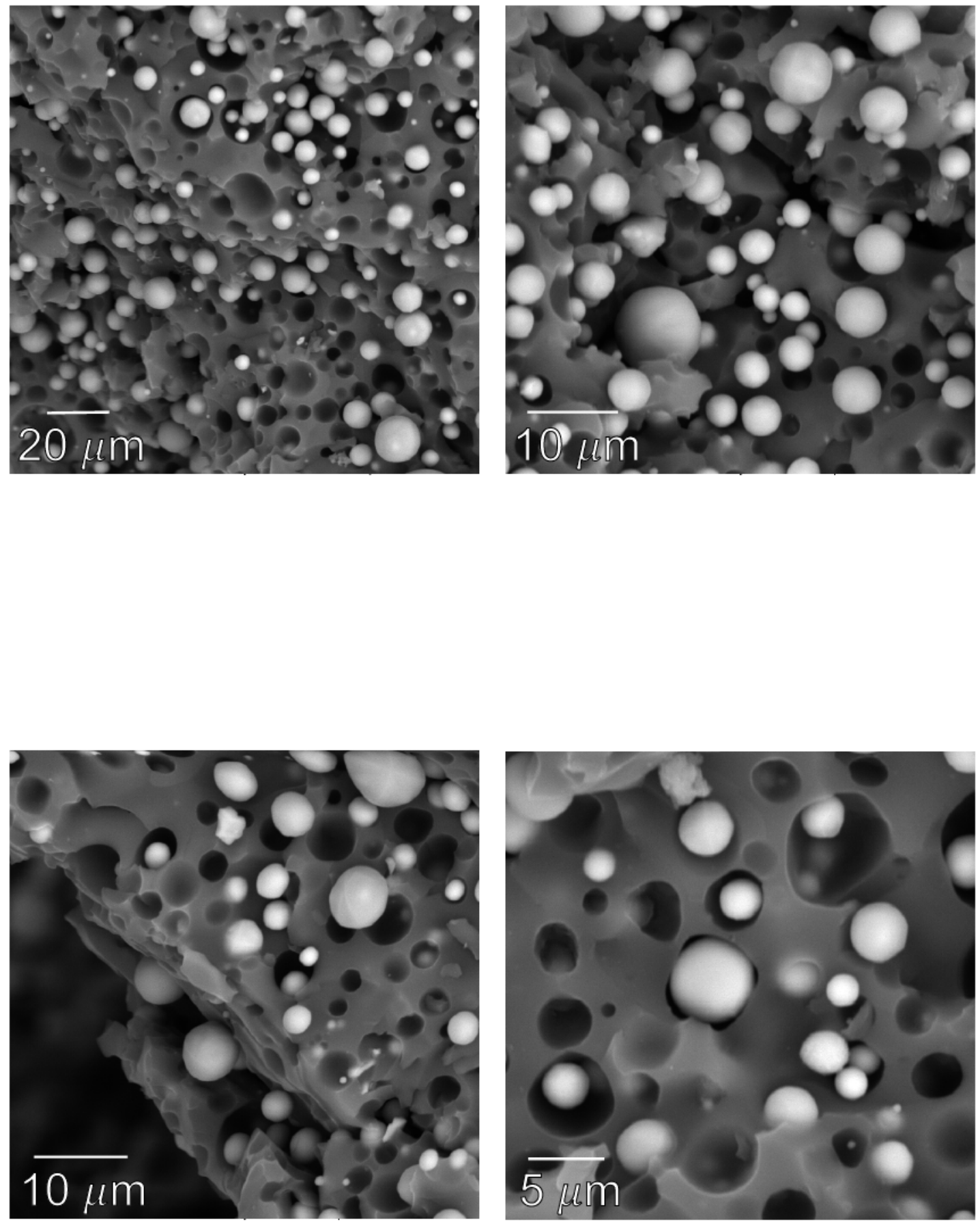


\section{C.5 Imaging of the Polyurethane-Silane Blended Samples}
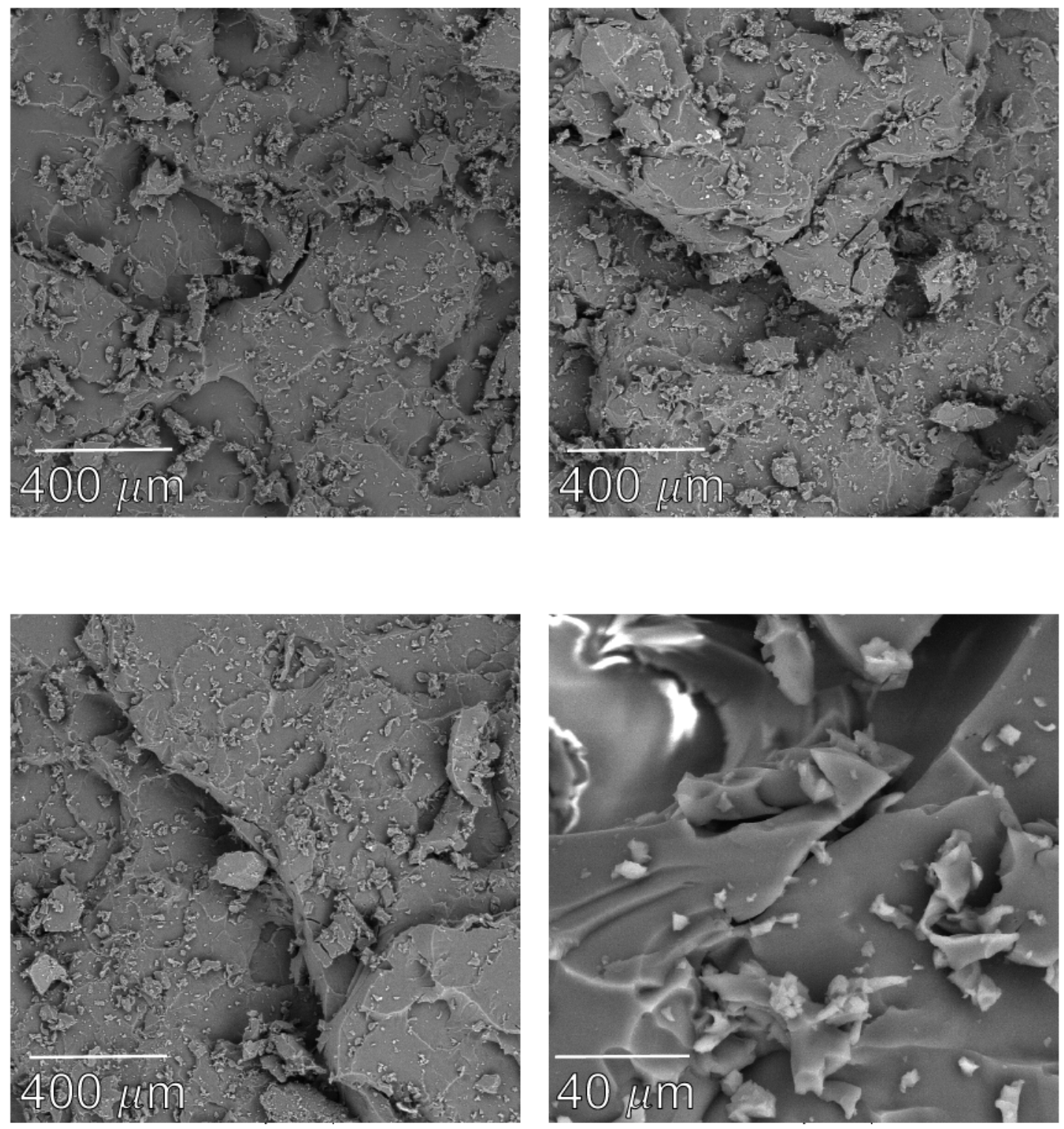

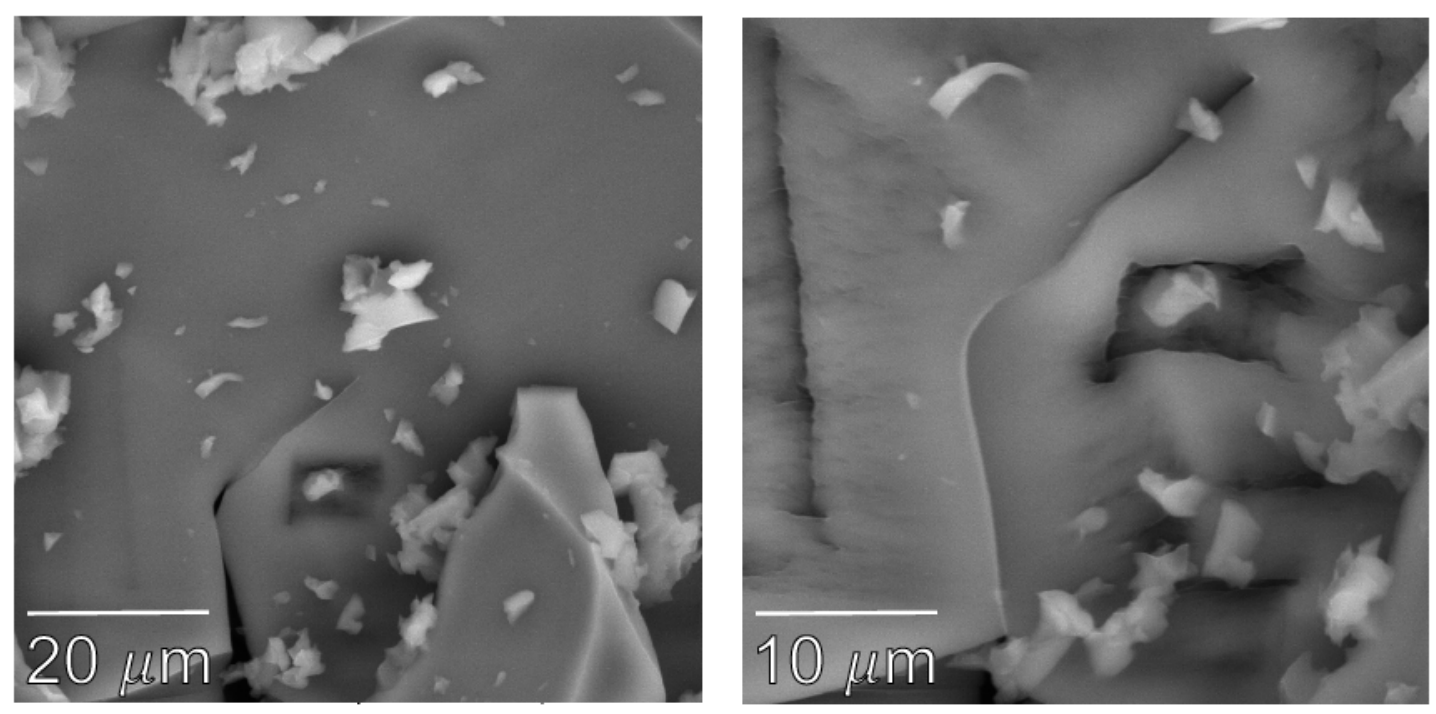


\section{Appendix D}

\section{Silane Chemistry}

In a topical sense silanes are to ceramics and polymers, as soaps are to polar (water) and non-polar (oils) chemicals. With its long molecule, capped with polar and covalent ends (functional groups), soap acts as a chemical intermediary. This allows water molecules to be bound to oil molecules via the soap molecule. This chemical bond allows for the removal of oils from dishes and textiles through mechanical forces in the washing process.

Silanes, like soap rely on a long molecule with two unique ends to facilitate bonding to the unique surfaces present on either end of the molecule, in this case ceramics and polymers. More specifically a silane molecule is made of a four elements: an organo-functional group, a $\left(\mathrm{CH}_{2}\right)_{n}$ hydrocarbon chain, a silicon atom, and one or more hydrolysable groups [79]. The organo-functional group is responsible for interacting with the polymer, while the hydrolysable group is responsible for bonding to the substrate (in this case the ceramic filler).

Bonds between the hydrolysable group and the ceramic filler form as a result of a reaction between hydroxyl groups present on both the silane and the ceramic substrate. The thorough and complete chemical mechanism is well explained by Arkles [80]. In a general sense a covalent bond is generated during the drying process between the hydroxyl group of the silane and the ceramic substrate with the concurrent generation of water from the loss of hydrogen. It is typical for only one of the silanol groups $(\mathrm{SiOH})$ in a silane molecule to bond with the remaining groups existing in condensed or free form states [80]. The presence of these remaining silanol groups can also be seen above in the free hydroxyl groups 
of the silane chain in the right side of Figure D.1. This bonding mechanism is supported by Su and Shi [81] who show $\mathrm{Si}-\mathrm{OH}$ bonding between silica, and a silane with the same functional group of $\mathrm{SI}-\mathrm{OH}-\mathrm{CH}_{2}-\mathrm{CH}_{3}$.
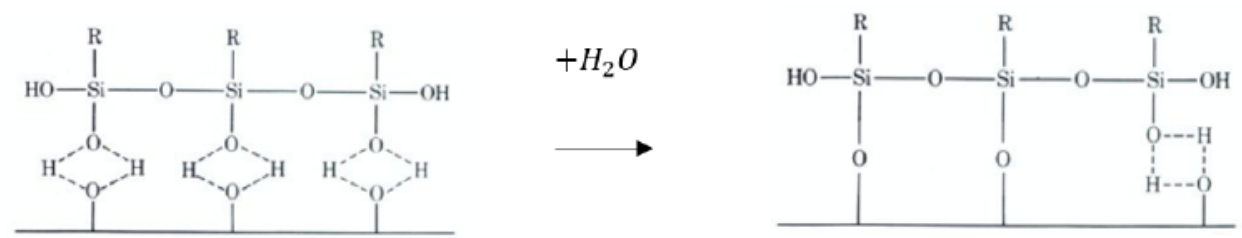

Figure D.1: Hydrolysis reaction schematic (Modified from [80]) 


\section{Appendix E}

\section{Data Processing Scripts}

All data processing performed in this study was performed using MATLAB R2016A.

\section{E.1 Read Lecroy Oscilliscope}

This is a publicly available script obtained to convert the binary data from the Lecroy brand oscilloscopes. The file "ReadLeCroyBinaryWaveform.m" is available with a straightforward internet search using the file name provided here. 


\section{E.2 Processing of Shock pin Data}

Script was ran twice with the first run used to identify the individual shock pin trigger times. The second run would calculate tilt based off of these tilts

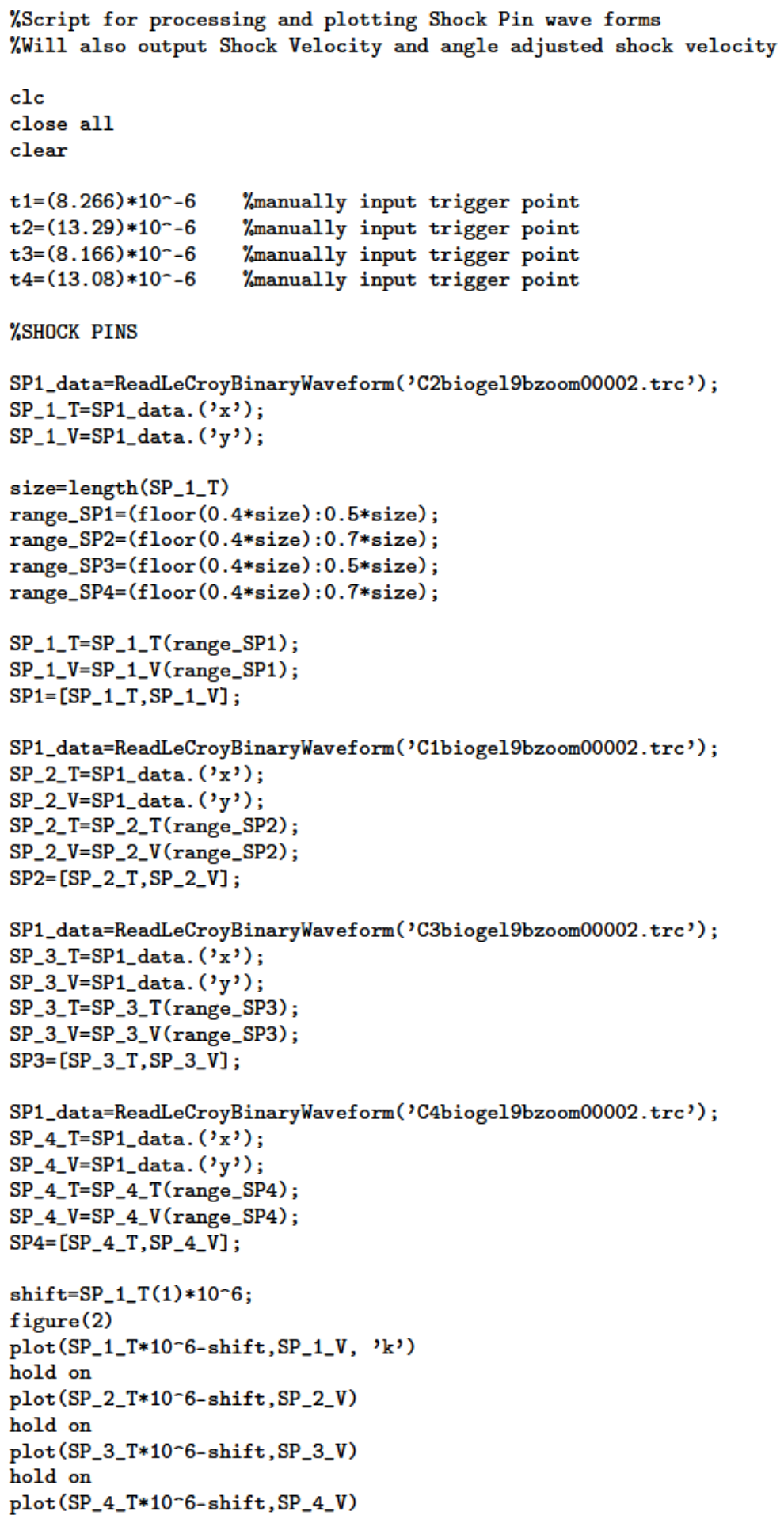


legend('3', ' ', ', ', '6')

xlabel('time (microsec)')

ylabel ('voltage (V)')

\%estimate angle

dy1_3=abs ( (v_CG)*(t3-t1));

theta=asind (dy1_3/0.011);

theta_mrad=asin $\left(\right.$ dy $\left.1 \_3 / 0.011\right) * 1000$

dy2_4=abs ( (v_CG)*(t2-t4));

phi=asind (dy2_4/0.011);

phi_mrad=asin (dy2_4/0.011)*1000

distance $=(1.089-.694) * .0254$;

$\mathrm{U}=$ distance $/(\operatorname{mean}([\mathrm{t} 2, \mathrm{t} 4])-\operatorname{mean}([\mathrm{t} 1, \mathrm{t} 3])) \quad \%$ if no angle

U_angle_adjusted $=($ distance $*$ sind $(90-$ theta $) * \operatorname{sind}(90-$ phi $)) \ldots$

$/(\operatorname{mean}([\mathrm{t} 2, \mathrm{t} 4])-\operatorname{mean}([\mathrm{t} 1, \mathrm{t} 3]))$ 


\section{E.3 Processing of Coil Gauge Data}

Script was ran twice with the first run used to find the Coil Gauge Cross Over times

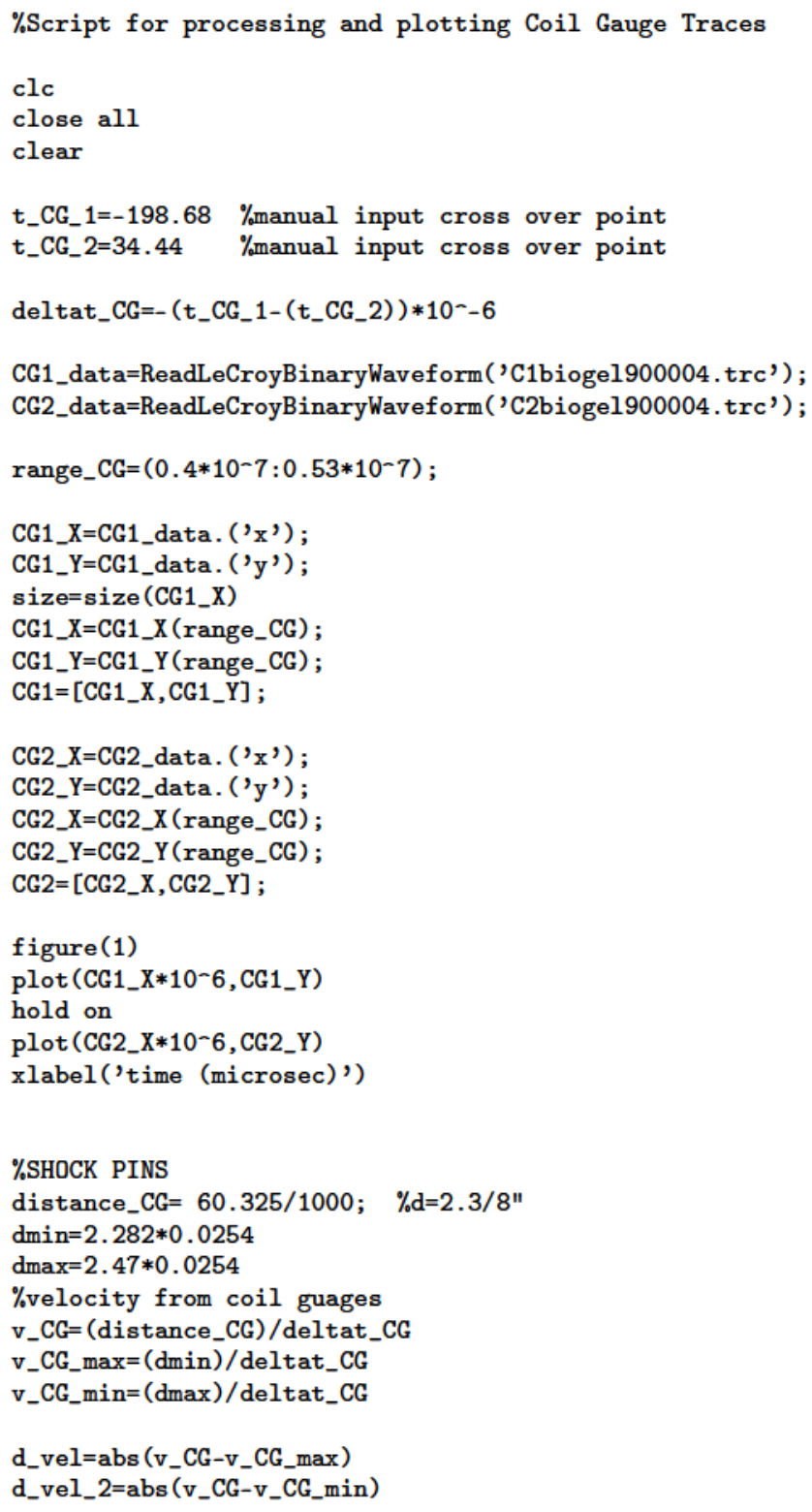




\section{E.4 Photo Doppler Velocimetry Processing}

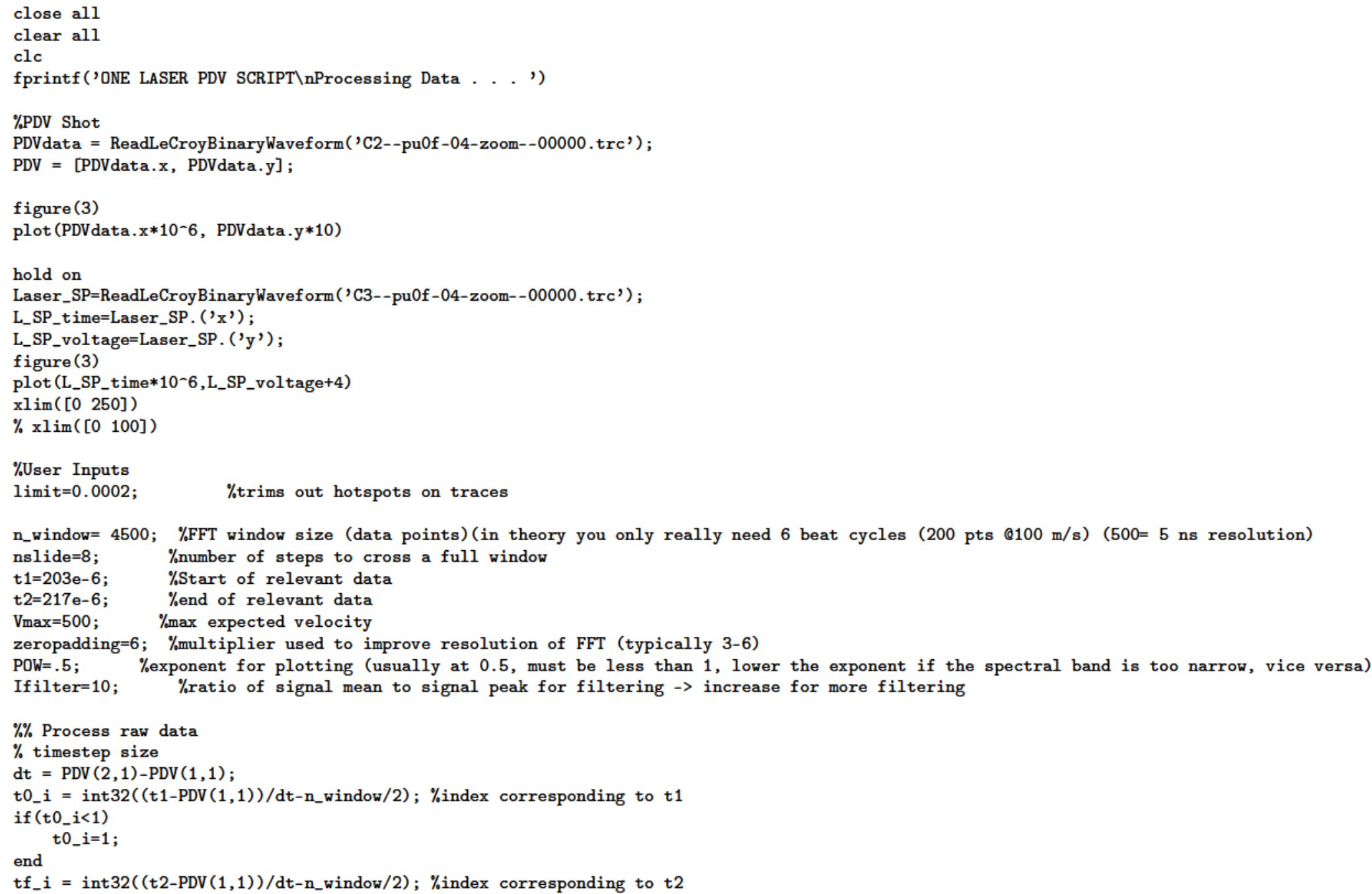


$t=P D V\left(t 0_{-} i(1): t f_{-} i(1), 1\right) ;$

$V=P D V\left(t 0_{-} i(1): t f_{-} i(1), 2\right)$;

$\%$ FFT parameters

$F s=1 /(t(2)-t(1)) ;$

NFFT $=$ n_window $*$ zeropadding;

$\%$ sampling frequency $[\mathrm{Hz}]$

$f=\mathrm{Fs} / 2 * \operatorname{linspace}(0,1, \mathrm{NFFT} / 2+1)$

w=hamming (n_window+1, 'periodic');

$\operatorname{Vel}(:, 1)=775 * \mathrm{f} / 1 \mathrm{e} 9$;

$\%$ zero padding

$\%$ frequency range vector (range: 0 to niquist frequency (FS/2), length:NFFT/2)

$\%$ hamming window function

$\%$ velocity vector corresponding to frequency range

$\mathrm{dV}=\mathrm{Vel}(2)-\mathrm{Vel}(1)$;

iimax $=i n t 32\left(V_{\max } /(\operatorname{Vel}(2)-\mathrm{Vel}(1))\right)+1 ; \%$ set $\max$ index corresponding to max velocity

$\%$ FFT loop

$i i=1$;

$j \mathrm{j}=1$;

while $\left(i i+t 0 \_i+n_{-}\right.$window $\left.t f_{-} i\right)$

$\mathrm{Y}=\mathrm{fft}\left(\mathrm{w} \cdot * \mathrm{~V}\left(\mathrm{ii}: \mathrm{i} i+\mathrm{n} \_\right.\right.$window, 1$\left.), \mathrm{NFFT}\right)$

$A=\operatorname{abs}(\mathrm{Y}(1: \mathrm{NFFT} / 2+1)) .-\mathrm{POW} / \mathrm{NFFT}$;

$\mathrm{kk}=1$;

while $(A(k k+1)<A(k k))$ \%ignore the low frequency noise in the FFT $\mathrm{kk}=\mathrm{kk}+1$;

end

iimin=kk;

$[B, C]=\max (A($ iimin: iimax $))$

$\operatorname{VelPDV}(j \mathrm{j}, 1)=\operatorname{Vel}(\mathrm{C}+\mathrm{iimin}-1)$;

$\operatorname{VelPDV}(j j, 2)=0.5 *\left(t(i i)+t\left(i i+n_{-} w i n d o w\right)\right) ;$

$\operatorname{VelPDV}(j \mathrm{j}, 3)=\mathrm{B}$;

$\operatorname{VelPDV}(j \mathrm{j}, 4)=\operatorname{mean}(A(\mathrm{imin}: \mathrm{i} \operatorname{imax}))$;

$i i=i i+i n t 32$ (n_window/nslide);

AA $(:, j j)=A(1:$ iimax $)$;

$j j=j j+1$;

end

$\%$ Filter the time-velocity curve

$j j \max =j j-1$;

$j \mathrm{j}=1$;

while $(j j<=j$ jmax $)$

if $(\operatorname{VelPDV}(j \mathrm{j}, 3)>$ If ilter $* \operatorname{VelPDV}(j \mathrm{j}, 4)$ )

$\operatorname{VelocityPDV}(\mathrm{hh}, 1)=\operatorname{VelPDV}(j \mathrm{j}, 1)$;

VelocityPDV $(h h, 2)=\operatorname{VelPDV}(j j, 2)$;

$\mathrm{hh}=\mathrm{hh}+1$;

end 
end

$\% \% \%$ ind displacement

\% VelocityPDV $(:, 3)=\operatorname{cumtrapz}(\operatorname{VelocityPDV}(:, 2), \operatorname{VelocityPDV}(:, 1))$;

fprintf ('\nCreating Plots...')

\%graph data

$\%$ \%Raw PDV Signal

$\%\{$

figure(1)

plot (PDV $(:, 1) * 1 e 6, \operatorname{PDV}(:, 2)$, 'b')

$\mathrm{a}=\mathrm{gca}$;

a. TickLabelInterpreter = 'latex';

a. Box = 'on';

a. BoxStyle = 'full';

ylabel ('Voltage (V)', 'Interpreter', 'latex')

xlabel('Time (\$\us \$s)', 'Interpreter','latex')

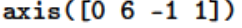

set (gca, 'fontsize', 12)

$\%\}$

\%Processed PDV Signal - Spectrogram

figure (2)

scale $=0: 0.1 * \max (\max (A A)): \max (\max (A A))$;

$\mathrm{AA}(\mathrm{AA}>$ limit $)=1$ imit;

set (gca, 'fontsize', 30)

contourf (VelPDV (: ,2) .*1e6, Vel (25: iimax) ,AA (25: iimax, : ) , 'LineColor', 'none')

colormap (jet)

$\% \mathrm{c}=$ colorbar

\% c.Label.string = 'Normalized Intensity';

\%shading flat

$\mathrm{a}=$ gca;

a. TickLabelInterpreter = 'latex';

a. Box = 'on';

a. BoxStyle = 'full';

xlabel('Time (\$\mu\$s)', 'Interpreter', 'latex');

ylabel ('Velocity (m/s)', 'Interpreter', 'latex');

$\% \operatorname{axis}\left(\left[\begin{array}{llll}0 & 6 & 50 & 1200\end{array}\right]\right)$

set (gca,' $f$ ontsize', 12)

$\%\{$

figure (3)

plot (VelPDV $(:, 2) . * 1 \mathrm{e} 6, \operatorname{VelPDV}(:, 1))$

hold on

xlabel ('Time (\$\mus)', 'Interpreter', 'latex'); 
ylabel ('Velocity (m/s)', 'Interpreter', 'latex');

hold off 


\section{E.5 Design of spall experiments}

The below code was used to predict spall plane location, as well as expected shock properties. It was used to justify the geometry used in this study

clc
clear

close all

\%impact info begin

flyer_plate_speed $=0.5$

$w_{-} f l$ yer $=(1 / 8) * 0.0254$;

w_target $=0.005$;

w_striker $=(1 / 8) * 0.0254$;

fly_mat $=2$;

target_mat $=3$;

striker $=0 \%$ no striker present $=0$, striker present $=0$

\%impact info end

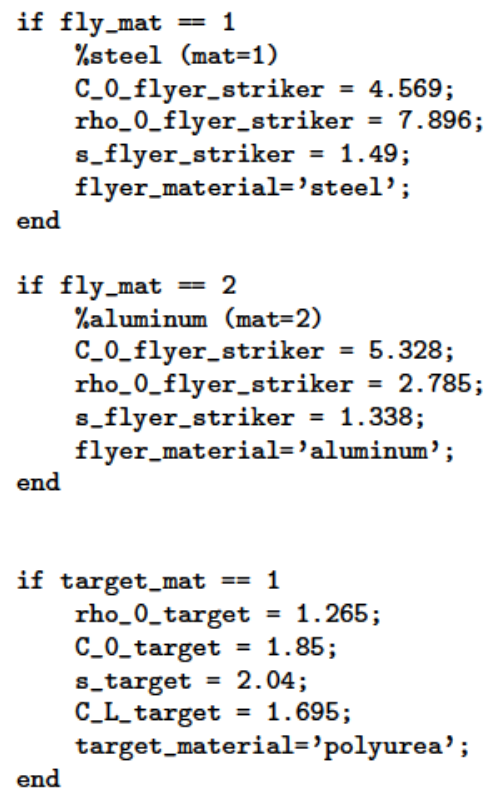

if target_mat $==1$

rho_0_target $=1.265$;

C_0_target $=1.85$;

s_target $=2.04$;

C_L_target $=1.695$;

target_material='polyurea';

end

if target_mat $==2$ 
rho_0_target $=1.265$;

C_0_target $=2.486$

s_target $=1.577$;

C_L_target $=1.8$;

end

target_material='Polyurethane';

if target_mat $==3$

rho_0_target $=1.1$

C_0_target $=1.265$;

s_target $=2.43$;

C_L_target $=1.8$;

target_material='PDMS';

if striker $=0$

\%Initial Impact Properties

[ P_1,u_1 ] = P_u_Interface(C_0_flyer_striker,rho_0_flyer_striker, s_flyer_striker,rho_0_target, C_0_target, s_target,flyer_plate_speed);

U_target=C_0_target+s_target*u_1;

U_flyer_striker=C_0_flyer_striker+u_1*s_flyer_striker;

t_backface_us=w_flyer/(U_flyer_striker*10-3);

t_freesurface_us=w_target/(U_target $* 10-3)$;

\%rarefaction in the flyer material, u_2 is freesurface velocity, u_1 is particle

$\%$ velocity from the shock wave

\% u_p added here because the u_p and U_rare are in the same directions so it

$\%$ considers that the wave is actually faster than it eularian velocity

u_2_flyer_striker=u_1-(flyer_plate_speed-u_1); \%goes to zero which makes sense for the RG Hugoniot

U_rare_backface_lag_RG=U_rare (C_0_flyer_striker,s_flyer_striker, u_1, u_2_flyer_striker)

U_rare_backface_eularian_RG=U_rare_backface_lag_RG+u_1;

U_rare_target_lag_RG=U_rare (C_0_target, s_target , u_1,0);

U_rare_target_eularian_RG=U_rare_target_lag_RG + u_1;

u_2_target=2*u_1;

\%rarefaction in the target, u_2 is freesurface velocity, u_1 is particle $\%$ velocity from the shock wave

\%math done in next line directly to account for LG effects

U_rare_free_surface_lag_LG=-C_0_target-2*s_target*(u_2_target-u_1);

$\% u_{-p}$ added here because the u_p and U_rare are opposite directions so it $\%$ considers that the wave is actually slower than it eularian velocity 
U_rare_free_surface_eularian_LG=U_rare_free_surface_lag_LG+u_1;

U_rare_free_surface_eularian_LG_abs=-U_rare_free_surface_eularian_LG ;

t_rare_interface_RG_us=t_backface_ust (w_flyer) /(U_rare_backface_eularian_RG*10-3);

t_rare_backface_RG_us=t_rare_interface_RG_us+(w_target)/(U_rare_target_eularian_RG*10-3);

t_rare_interface_LG_us=t_freesurface_ust (w_target) / (U_rare_free_surface_eularian_LG_abs*10-3);

\%use $\mathrm{C}_{-} \mathrm{O}$ as velocity because the U_rare equation goes to $\mathrm{C}_{-} 0$ as $\mathrm{u}_{-} 1-\mathrm{u}_{-} 2$ \%goes to 0.

t_rare_min_RG_BF_INT= t_backface_ustw_flyer/(C_0_flyer_striker*1000);

t_rare_min_RG_INT_FS= t_rare_min_RG_BF_INT+ w_target/(C_0_target*1000);

t_rare_min_LG_BF_INT $=$ t_freesurface_ustw_target/(C_0_target $* 1000)$;

\section{figure()}

\%interfaces

plot $([0,0],[0$, t_rare_interface_LG_us $]$,'black')

hold on

plot ([-w_flyer*10-3,-w_flyer*10-3] , [0,t_rare_interface_LG_us] , 'black')

hold on

hold on

plot ([(w_target*10-3), (w_target*10-3)], [0,t_rare_interface_LG_us] , 'black')

hold on

$\%$ shocks

plot ( [0,-w_flyer*10-3] , [0,t_backface_us] , 'red')

hold on

plot ([0,w_target*10-3] , [0,t_freesurface_us] ,'red')

hold on

$\%$ rarefactions

hold on

plot ([-w_flyer*10-3, 0] , [t_backface_us, t_rare_interface_RG_us] , 'blue')

hold on

plot ( [0,w_target*10-3] , [t_rare_interface_RG_us,t_rare_backface_RG_us] , 'blue')

hold on

plot ([(+w_target*10-3), 0], [t_freesurface_us, t_rare_interface_LG_us] , 'blue')

hold on

plot ([-w_flyer*10-3,0], [t_backface_us, t_rare_min_RG_BF_INT] ,'--blue')

hold on

plot ([0,w_target*10-3] , [t_rare_min_RG_BF_INT,t_rare_min_RG_INT_FS] , '--blue')

hold on 
plot ([(+w_target*10-3), 0], [t_freesurface_us, t_rare_min_LG_BF_INT] , '--blue')

xlabel ('X axis (mm)')

ylabel ('time(s)')

timelimit_crop=t_rare_interface_LG_us;

ylim([0 timelimit_crop $]$ );

end

P_tensile $=$ rho_0_target*C_L_target*(-u_1) \%check;

\%Belows considers the existance of a striker plate

if striker $=1$

\%partice velocity after symetrical impact

u_1 = flyer_plate_speed $/ 2$;

P_1=rho_0_flyer_striker*C_0_flyer_striker*u_1+rho_0_flyer_striker*s_flyer_striker*u_1-2;

\%shock wave velocity

U_shock_flyer_striker = C_0_flyer_striker+u_1*s_flyer_striker;

$\%$ Striker-PDMS interface

$\% u 0_{-}$steel gives the $\mathrm{x}_{-}$intercept of the left going Pu hugoniot and is equal

$\%$ to the particle velocity resulting from the initial impact

[P_PDMS, u_PDMS] = P_u_Interface (rho_0_flyer_striker,C_0_flyer_striker, s_flyer_striker, rho_0_target, C_0_target, s_target,flyer_plate_speed); U_target $=$ C_0_targettu_PDMS*s_target;

t_backface_us=w_flyer/(U_shock_flyer_striker*10-3);

t_interface_us=w_striker/(U_shock_flyer_striker*10-3);

t_freesurface_us=t_interface_us+w_target/(U_target*10-3);

u_2_flyer_striker $=0$;

U_rare_backface_lag_RG=U_rare (C_0_flyer_striker, s_flyer_striker, u_1, u_2_flyer_striker);

U_rare_backface_eularian_RG=U_rare_backf ace_lag_RG+u_1;

U_rare_target_lag_RG=U_rare (C_0_target, s_target, u_PDMS , 0);

U_rare_target_eularian_RG=U_rare_target_lag_RG + u_PDMS;

t_rare_interface_RG_us=t_backface_ust (w_flyer+w_striker) /(U_rare_backface_eularian_RG*10-3) ;

t_rare_backface_RG_us=t_rare_interface_RG_us+(w_target)/(U_rare_target_eularian_RG*10-3);

u_2_target=2*u_PDMS ;

U_rare_free_surface_lag_LG=-C_0_target-2*s_target*(u_2_target-u_PDMS); 
U_rare_free_surface_eularian_LG=U_rare_free_surface_lag_LG+u_PDMS;

U_rare_free_surface_eularian_LG_abs=-U_rare_free_surface_eularian_LG ;

t_rare_interface_LG_us=t_freesurface_ust (w_target) / (U_rare_free_surface_eularian_LG_abs*10-3) ;

figure()

plot $\left([0,0],\left[0, t_{-}\right.\right.$rare_interface_LG_us $]$,'black')

hold on

plot ([-w_flyer*10-3,-w_flyer*10-3], [0,t_rare_interface_LG_us], 'black')

hold on

plot ([w_striker*10-3,w_striker*10-3] , [0,t_rare_interf ace_LG_us] , 'black'

hold on

plot ([(w_striker*10-3+w_target*10-3), (w_striker*10-3+w_target*10-3)], [0,t_rare_interface_LG_us] , 'black')

hold on

$\%$ shocks

plot ( [0,-w_flyer*10-3] , [0,t_backface_us] ,'red')

hold on

plot ([0,w_striker*10-3] , [0,t_interface_us] , 'blue')

hold on

plot ([w_striker*10-3, (w_striker*10-3+w_target*10-3)] , [t_interface_us , t_freesurface_us] , 'blue')

$\%$ rarefactions

hold on

plot ([-w_flyer*10-3,w_striker*10-3] , [t_backface_us,t_rare_interface_RG_us] , 'red')

hold on

plot ([w_striker*10-3, (w_striker*10-3+w_target*10-3)], [t_rare_interface_RG_us,t_rare_backface_RG_us] , 'red')

hold on

plot ([(w_striker*10-3+w_target*10-3), w_striker*10-3] , [t_freesurface_us, t_rare_interface_LG_us] , 'blue')

end

P_tensile $=$ rho_0_target*C_L_target $*(-$ u_PDMS $)$

flyer_material

target_material

\section{E.5.1 P_u_Interface}

Used to determine end state when a shock wave passes an interface seperating two materials of different impedances

function [ P_b,u_b ] = P_u_Interface ( rho_0_a, C_0_a, s_a,rho_0_b, C_0_b, s_b,u0_a)

$\%$ P_u_Interface A defines the incident material, B defines the material the

\%wave is transmitted into. Has been validated using Cooper, Eg. 18.1,18.2,18.3

$\%$ Detailed explanation goes here 
a1=rho_0_b*s_b-rho_0_a*s_a;

b1 $=$ rho_0_b*C_0_b+(rho_0_a*C_0_a ) $2 *$ rho_0_a*s_a*u0_a;

$c 1=-r h 0_{-} 0_{-} a * C_{-} 0_{-} a * u 0_{-} a-\left(r h o_{-} 0_{-} a * s_{-} a * u 0_{-} a-2\right)$;

quadratic2 $=\operatorname{roots}([a 1, b 1, c 1])$

u_b=quadratic2(2);

u_b_1=quadratic2(1)

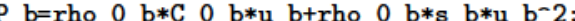

figure()

pu_b $=Q(u)$ rho_0_b*C_0_b*u+rho_0_b*s_b*u. ${ }^{-2}$;

fplot (Q(u) pu_b(u), [0,1.1*u_b],'LineWidth',3)

hold on

pu_a $=Q(u)$ rho_0_a*C_0_a*u+rho_0_a*s_a*u. -2

$\%$ fplot (@(u) pu_a(u), [0,u0_a], 'LineWidth', 3)

hold on

pu_a_flipped $=Q(u)$ rho_0_a*C_0_a*(u0_a-u) +rho_0_a*s_a*(u0_a-u).-2 ;

fplot $(\Theta(u)$ pu_a_flipped (u) , [(10/11)*u_b, u0_a] , 'LineWidth',3)

hold on

plot (u_b,P_b, '*', 'LineWidth',10)

xlabel ('particle velocity $(\mathrm{km} / \mathrm{s})$ ')

ylabel ('Pressure (GPa)')

\%text(u_b,P_b, 'lleftarrow Shocked State', 'FontSize',12)

\section{E.5.2 U_rare}

function $[\mathrm{U}]=\mathrm{U}_{-} \operatorname{rare}\left(\mathrm{C}_{-} 0, \mathrm{~s}_{\mathrm{u}} \mathrm{u}_{-}\right.$1, u_2 $)$

$\%$ U_rare gives the Lagrangian wave speed of rarefaction (Eq. 19.2 Cooper)

\% Detailed explanation goes here

$\mathrm{U}=\mathrm{C} \_0+2 * \mathrm{~s} *\left(\mathrm{u}_{-} 1-\mathrm{u}_{-} 2\right)$;

end 